Florida International University FIU Digital Commons

3-28-2013

\title{
Multifunctional Nanoparticles in Cancer: in vitro Characterization, in vivo Distribution
}

Tingjun Lei

Florida International University, tlei002@fiu.edu

DOI: $10.25148 /$ etd.FI13042501

Follow this and additional works at: https://digitalcommons.fiu.edu/etd

Part of the Bioimaging and Biomedical Optics Commons, Biomaterials Commons, Biotechnology Commons, Molecular, Cellular, and Tissue Engineering Commons, and the Nanoscience and Nanotechnology Commons

\section{Recommended Citation}

Lei, Tingjun, "Multifunctional Nanoparticles in Cancer: in vitro Characterization, in vivo Distribution" (2013). FIU Electronic Theses and Dissertations. 872.

https://digitalcommons.fiu.edu/etd/872

This work is brought to you for free and open access by the University Graduate School at FIU Digital Commons. It has been accepted for inclusion in FIU Electronic Theses and Dissertations by an authorized administrator of FIU Digital Commons. For more information, please contact dcc@fiu.edu. 


\section{FLORIDA INTERNATIONAL UNIVERSITY}

Miami, Florida

\section{MULTIFUNCTIONAL NANOPARTICLES IN CANCER: IN VITRO \\ CHARACTERIZATION, IN VIVO DISTRIBUTION, AND CELLULAR RESPONSE \\ AFTER LASER-NIR DYE-INDUCED HEATING}

A dissertation submitted in partial fulfillment of

the requirements for the degree of

DOCTOR OF PHILOSOPHY

in

BIOMEDICAL ENGINEERING

by

Tingjun Lei

2013 
To: Dean Amir Mirmiran

College of Engineering and Computing

This dissertation, written by Tingjun Lei, and entitled Multifunctional Nanoparticles in Cancer: in vitro Characterization, in vivo Distribution, and Cellular Response after LaserNIR Dye-induced Heating, having been approved in respect to style and intellectual content, is referred to you for judgment.

We have read this dissertation and recommend that it be approved.

$\begin{array}{r}\hline \text { Yen-Chih Huang } \\ \hline \text { Joong-ho Moon } \\ \hline \text { Wei-Chiang Lin } \\ \hline \text { Anthony J. McGoron, Major Professor }\end{array}$

Date of Defense: March 28, 2013

The dissertation of Tingjun Lei is approved.

Dean Amir Mirmiran College of Engineering and Computing

Dean Lakshmi N. Reddi University Graduate School

Florida International University, 2013 
(C) Copyright 2013 by Tingjun Lei

All rights reserved. 


\section{DEDICATION}

I want to dedicate this dissertation to my wife $\mathrm{Yu} \mathrm{Bi}$, my parents and my grandma, who have patiently stayed and helped me throughout this journey. Especially my wife, she married me in the last year of my Ph.D., when I was really busy with doing experiments and writing my dissertation. Thanks for her understanding and all her efforts, which help me overcome all obstacles that I have come across during my Ph.D. I have to admit that I am not a very good husband since many times I cannot be with my wife when she needs me, but have to stay at school working on papers and doing experiments. I have to also admit that I am not a very good son either by leaving my parents in China and only seeing them once a year or even longer. However, my wife and my parents they are always there and are being patient with me. They truly gave me unconditional support and I could never repay their love and everything they gave me. I would like to also thank my grandma who took care of me when I was a child. She passed away during the time when I was writing my dissertation. I really miss her and if it is possible, I want to show her my doctorate degree diploma and hope that she will be proud of me.

These years Ph.D. life make me realize that where there is a will there is a way. I know that I am not only earning a doctoral degree, but I am also learning a life attitude. I understand now life will treat you the way you treat life. I will use these years' experience to educate my children in the future when they are thinking of giving up. I will tell them how their father finished his Ph.D. Last but not least, I want to thank all my other family members, I hope I have made you all proud. 
谢谢我的太太，我的父母，我的奶奶，我的家庭，没有你们，我不会有今天

\section{的成就，你们是我这辈子最重要的人!}

\section{ACKNOWLEDGMENTS}

I would like to acknowledge my major professor Dr. Anthony J. McGoron, for his guidance and mentoring. He always pushed me by asking me to give presentation in every lab meeting and raised many tough questions after I presented since he wanted me to think critically and get the best out of me. One of the things I learned from him is always criticizing yourself. He showed me the way of being a successful scientist and I really appreciate all his guidance throughout my Ph.D. I am also very grateful to my former and current lab members, especially Romila Manchanda, Yuan Tang and Alicia Fernandez-Fernandez for their help. Romila, as being a former Research Assistant Professor in our department, had guided me and helped me achieving my Ph.D. She was the producer of all the particles we used in our lab, without her, I will not be able to learn how to make nanoparticles and finish my dissertation. Yuan Tang and Alicia FernandezFernandez, as being former graduate Ph.D. students from our lab, have taught me all the techniques required for cell culture and animal studies. I have to say my accomplishment is established on their achievements. I also want to thank all my dissertation committees: Dr. Huang for providing me the polymers he developed in his lab; Dr. Moon for sharing with me his chemistry expertise; and Dr. Lin for giving me advice for biomedical optics. Thanks to them serving as my dissertation committee members and providing me with their feedback and questions to improve my dissertation. In addition, I want to thank Dr. Byrne to give me advice and always help me fix the instruments in my lab. Whenever 
there is an instrument problem, the first person I would think of to ask is Dr. Byrne. I also want to thank Dr. Fan for giving me access to the confocal microscope and being really nice to show me how to use the equipment; and Dr. Liu for helping me get the SEM images. In the end, I have to thank all my friends: Chang Liu, Xuena Zhu, Yinchen Song, Yi Ding, Xizi Dai, Dali Sun, Jiali Wang, Lu Wang, Tao Wang, Zhiqi Zhang, Li Fan, Linna Tang, Guangnan Deng, Yuanyuan Zhang and Huang Huang. They made my life wonderful.

Financial support during my Ph.D. studies was provided by a Teaching Assistantship from the Biomedical Engineering Department at FIU from 2007-2012, by a Research Assistantship from Dr. Anthony J. McGoron's grant project funding from 20122013. 


\author{
ABSTRACT OF THE DISSERTATION \\ MULTIFUNCTIONAL NANOPARTICLES IN CANCER: IN VITRO \\ CHARACTERIZATION, IN VIVO DISTRIBUTION, AND CELLULAR RESPONSE \\ AFTER LASER-NIR DYE-INDUCED HEATING
}

by

Tingjun Lei

Florida International University, 2013

Miami, Florida

Professor Anthony J. McGoron, Major Professor

A novel biocompatible and biodegradable polymer, termed poly(Glycerol malate cododecanedioate) (PGMD), was prepared by thermal condensation method and used for fabrication of nanoparticles (NPs). PGMD NPs were prepared using the single oil emulsion technique and loaded with an imaging/hyperthermia agent (IR820) and a chemotherapeutic agent (doxorubicin, DOX). The size of the void PGMD NPs, IR820PGMD NPs and DOX-IR820-PGMD NPs were approximately $90 \mathrm{~nm}, 110 \mathrm{~nm}$, and 125 nm respectively. An acidic environment $(\mathrm{pH}=5.0)$ induced higher DOX and IR820 release compared to $\mathrm{pH}=7.4$. DOX release was also enhanced by exposure to laser, which increased the temperature to $42^{\circ} \mathrm{C}$. Cytotoxicity of DOX-IR820-PGMD NPs was comparable in MES-SA but was higher in Dx5 cells compared to free DOX plus IR820 $(p<0.05)$. The combination of hyperthermia (HT) and chemotherapy improved 
cytotoxicity in both cell lines. We also explored the cellular response after rapid, shortterm and low thermal dose (laser/Dye/NP) induced-heating, and compared it to slow, long-term and high thermal dose cell incubator heating by investigating the reactive oxygen species (ROS) level, hypoxia-inducible factor-1 $\alpha$ (HIF-1 $\alpha$ ) and vascular endothelial growth factor (VEGF) expression. The cytotoxicity of IR820-PGMD NPs after laser/Dye/NP HT resulted in higher cancer cell killing compared to incubator HT. ROS level, HIF-1 $\alpha$ and VEGF expression were elevated under incubator HT, while maintained at the baseline level under the laser/Dye/NP HT. In vivo mouse studies showed that NP formulation significantly improved the plasma half-life of IR820 after tail vein injection. Significant lower IR820 content was observed in kidney in DOXIR820-PGMD NP treatment as compared to free IR820 treatment in our biodistribution studies $(\mathrm{p}<0.05)$. In conclusion, both IR820-PGMD NPs and DOX-IR820-PGMD NPs were successfully developed and used for both imaging and therapeutic purposes. Rapid and short-term laser/Dye/NP HT, with a low thermal dose, did not up-regulate HIF-1 $\alpha$ and VEGF expression, whereas slow and long-term incubator HT, with a high thermal dose, can enhance expression of both HIF-1 $\alpha$ and VEGF. 


\section{TABLE OF CONTENTS}

CHAPTER PAGE

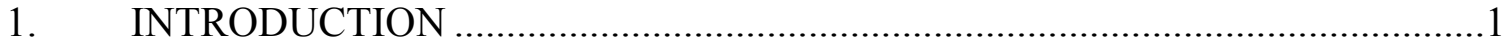

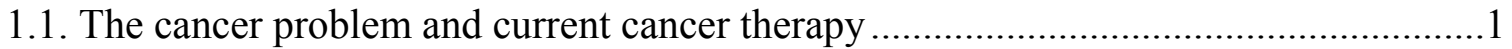

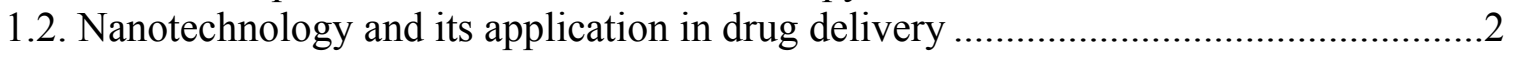

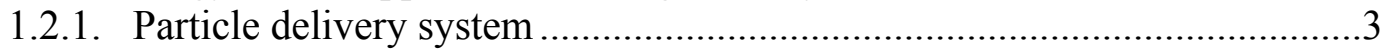

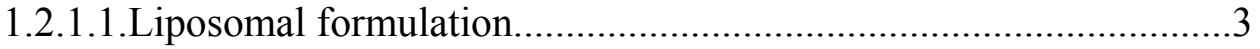

1.2.1.2.Nanoparticles delivery systems...............................................4

1.2.1.2.1. Naturally derived polymers...........................................5

1.2.1.2.2. Synthetic polymers........................................................

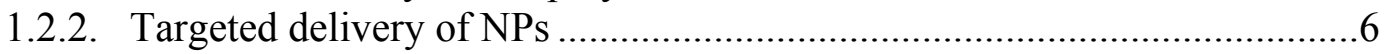

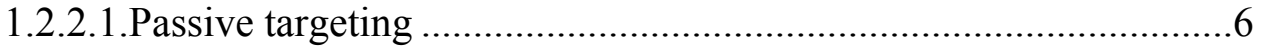

1.2.2.2. Active targeting.................................................................. 7

1.2.3. The development of multifunctional NPs .................................................

1.3. Novel polymeric NPs poly(glycerol-dodecanoate) (PGD) ......................................11

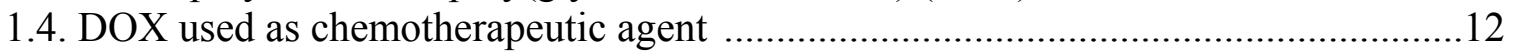

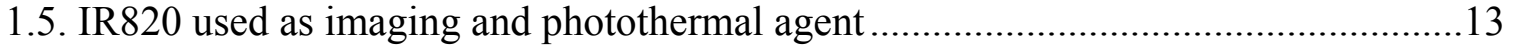

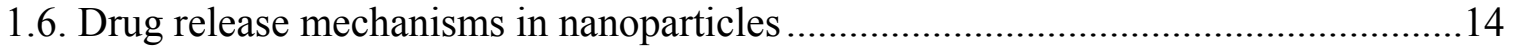

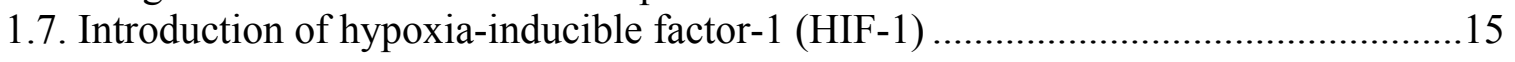

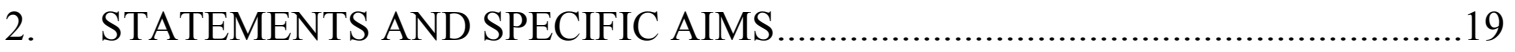

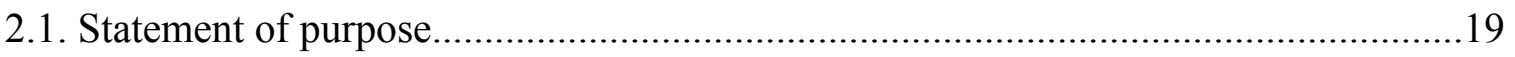

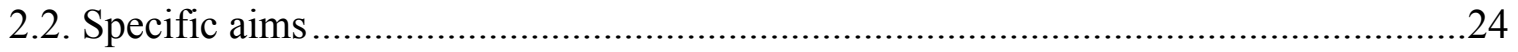

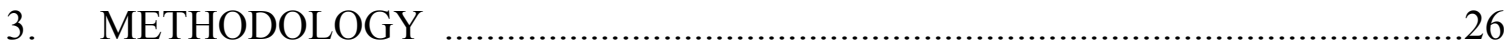

3.1. The fulfillment of Specific Aim 1 .........................................................................26

3.1.1. Preparation and characterization of PGMD polymer, void PGMD NPs,

IR820-PGMD NPs and DOX-IR820- PGMD NPs...............................26

3.1.2. Characterization of PGMD polymer, void PGMD NPs, IR820-PGMD NPs

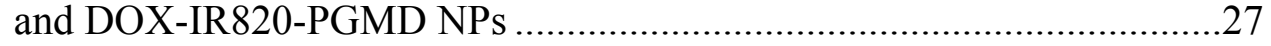

3.1.2.1. Characterization of PGMD polymer ..........................................27

3.1.2.2.NPs size, size distribution and zeta potential..............................27

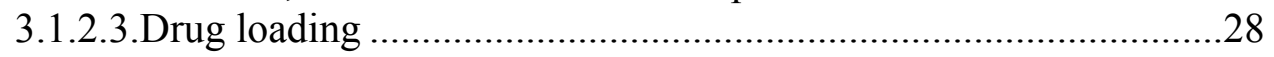

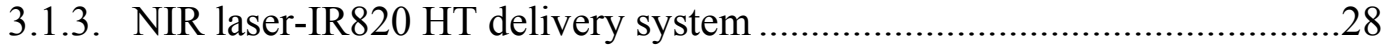

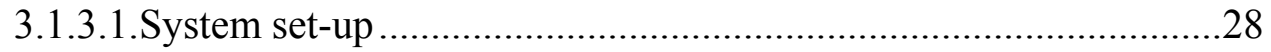

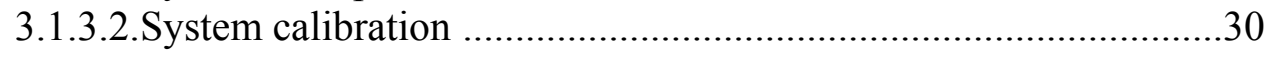


3.1.3.3.Heating capacity of free IR 820 and IR 820 in IR820-PGMD NPs

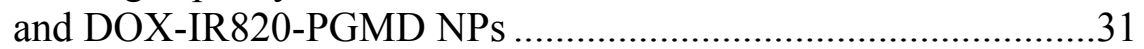

3.1.4. Incubator-induced HT and temperature profile ........................................32

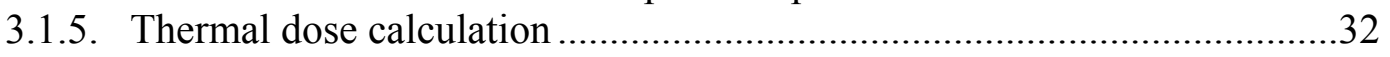

3.1.6. In vitro studies of void PGMD NPs, IR820-PGMD NPs and DOX-IR820-

PGMD NPs ……..........................................................................

3.1.6.1.IR820 and DOX release kinetics profile from DOX-IR820-PGMD

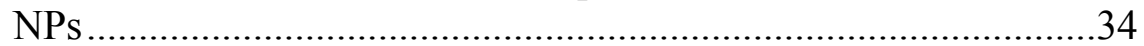

3.1.6.2.IR820 release kinetics profile in IR820-PGMD NPs.......................35

3.1.6.3.DOX cellular uptake experiment .....................................................35

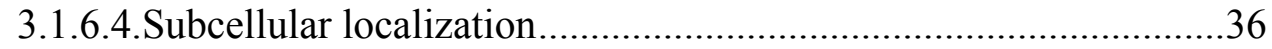

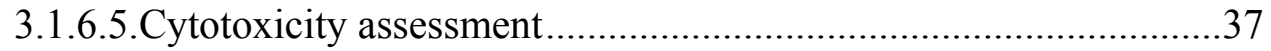

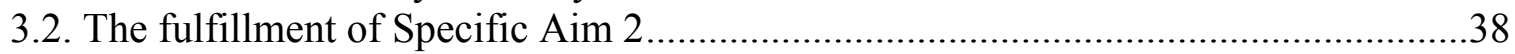

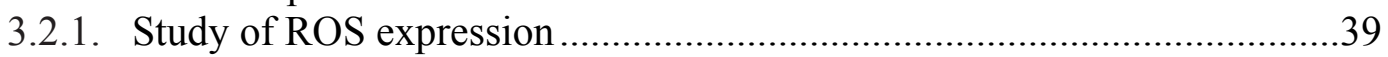

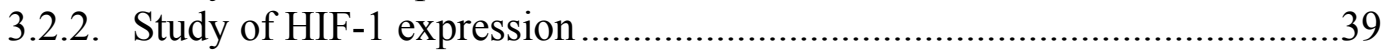

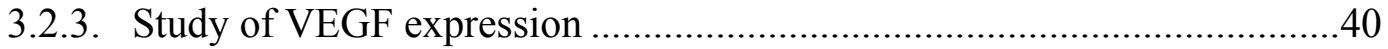

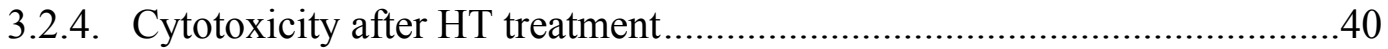

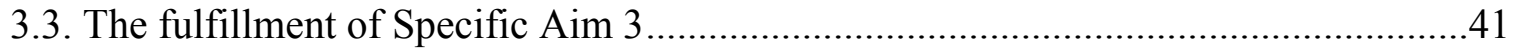

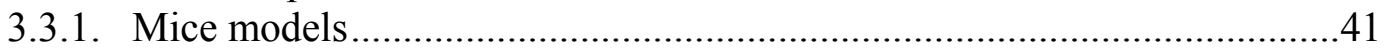

3.3.2. NIR in vivo biodistribution imaging ...................................................4

3.3.3. Pharmacokinetics and biodistribution study ……....................................43

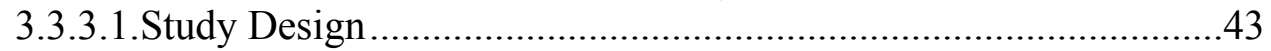

3.3.3.2.Pharmacokinetics study .................................................................4

3.3.3.3.Pharmacokinetic analysis of plasma data ......................................46

3.3.3.4. Quantitative measurement of organ content ..................................48

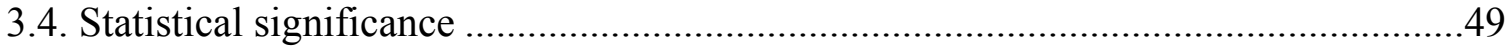

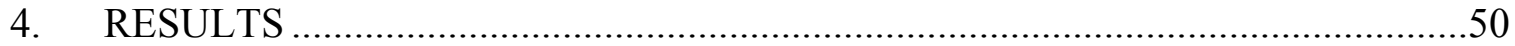

4.1. Results accomplished for Specific Aim 1 .........................................................50

4.1.1. Characterization of PGMD polymer .....................................................50

4.1.2. Characterization of void PGMD NPs, IR820-PGMD NPs and DOX-

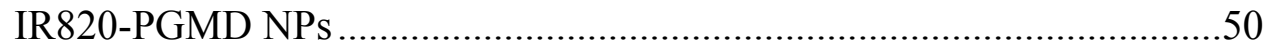

4.1.3. Heating modalities ...........................................................................52

4.1.3.1. Incubator HT delivery system and temperature increase profile...52

4.1.3.2. Laser-IR820-PGMD NPs HT delivery system and temperature increase profile .........................................................................53

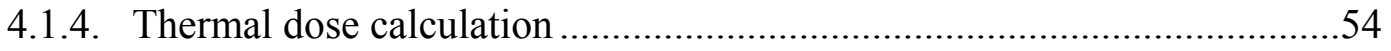

4.1.5. The in vitro studies of void PGMD NPs, IR820-PGMD NPs and DOX-

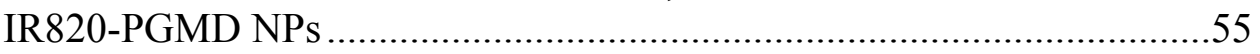

4.1.5.1. In vitro drug release ......................................................................5

4.1.5.2.DOX cellular uptake experiments................................................57

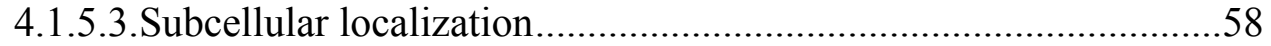

4.1.5.4.In vitro cytotoxicity.....................................................................62

4.1.5.4.1. Void PGMD NPs cytotoxicity profile.................................62

4.1.5.4.2. Cytotoxicity of IR820-PGMD NPs....................................63 


\subsection{Cytotoxicity of IR820-PGMD NPs, DOX-IR820-PGMD}

NPs with exposure to laser as compared to free IR820 and free DOX + IR820 ...............................................63

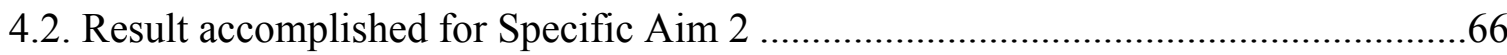

4.2.1. Cellular response after laser/Dye/NPs HT and incubator HT ...................66

4.2.1.1.ROS production after HT treatment.........................................66

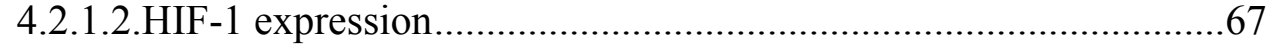

4.2.1.3.VEGF expression ................................................................68

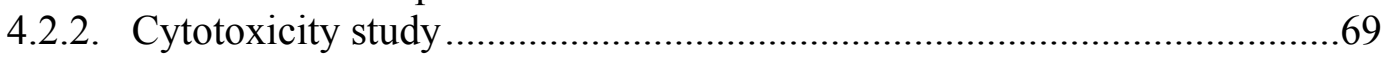

4.3. Results accomplished for Specific Aim 3 ........................................................ 71

4.3.1. Biodistribution and pharmacokinetic studies of IR820-PGMD NPs and

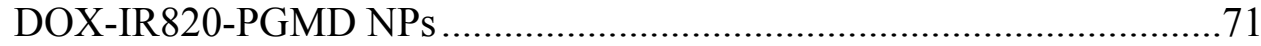

4.3.1.1.In vivo biodistribution imaging ............................................. 71

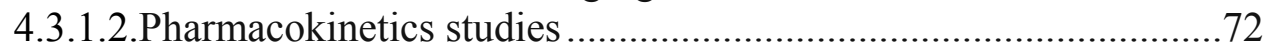

4.3.1.3.IR820 Pharmacokinetic modeling...........................................74

4.3.1.4.DOX pharmacokinetics modeling............................................77

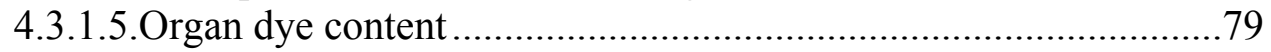

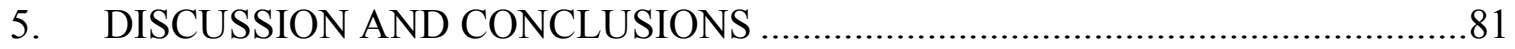

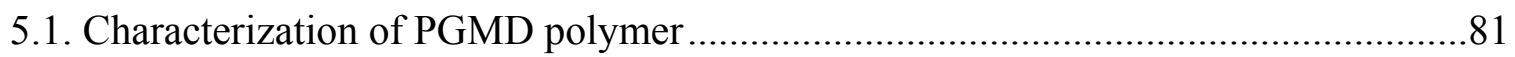

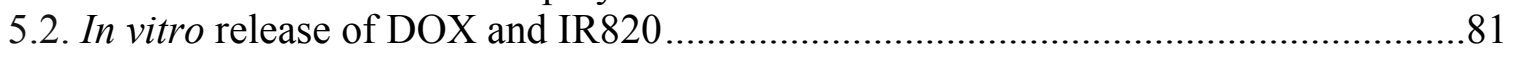

5.3. Subcellular localization of DOX and IR820 in DOX-IR820-PGMD-NPs and uptake

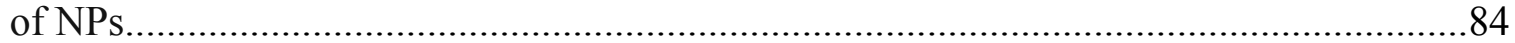

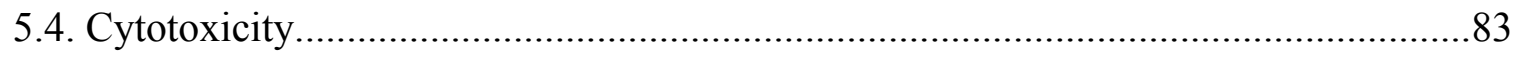

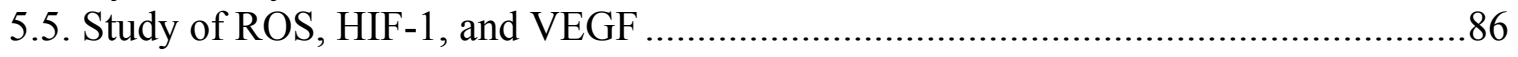

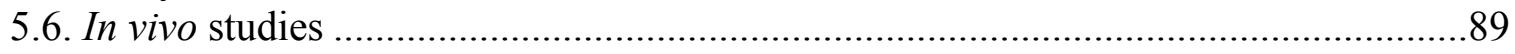

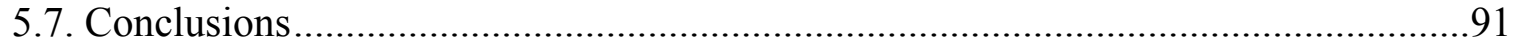

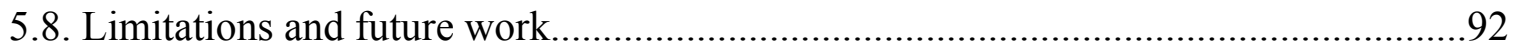

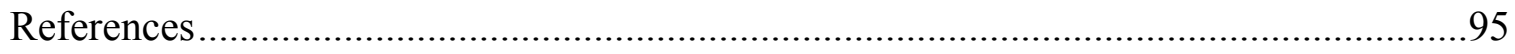

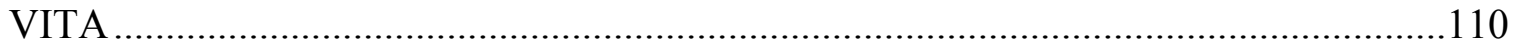




\section{LIST OF TABLES}

TABLE

PAGE

Table 1. A 96-well plate temperature distribution when placed on the heated stage insert.31

Table 2. Schematic of IR820 study design for measuring temperature profile in a 96-well plate $(n=3)$.

Table 3. Study design for biodistribution experiments of IR820, IR820-PGMD NPs, and DOX-IR820-PGMD NPs.

Table 4. Mean size, PDI, zeta potential, and percent of loading efficiencies for void

PGMD NPs, IR820-PGMD NPs, and DOX-IR820-PGMD NPs $(\mathrm{n}=8)$

Table 5. Calculated $\mathrm{CEM}_{42}$ in different types of HT treatment, based on the temperature curves in Figures 4 and 5 and using Equations 1 and 2.

Table 6. Image ratio $\mathrm{R}$ was calculated from fluorescence images of free IR 820 and IR820-PGMD NPs treatments $(n=3)$.

Table 7. Plasma concentrations ( $\mu \mathrm{g} / \mathrm{mL})$ of IR820, IR820-PGMD NPs and DOX-IR820-

PGMD NPs at different time points after injection

Table 8. Plasma concentrations ( $\mu \mathrm{g} / \mathrm{mL})$ of DOX in DOX-IR820-PGMD NPs at different time points after injection ...................................................................... 74

Table 9. Goodness of fit by using one-compartment model.......................................75

Table 10. Goodness of fit by using two-compartment model......................................76

Table 11. Pharmacokinetic parameters calculated based on one-compartment analysis of dye data in mice.

Table 12. Goodness of fit by using one or two-compartment model. .78

Table 13. Pharmacokinetic parameters from two-compartment analysis of dye data in mice. 
Table 14. Quantitative organ content 24 hours after i.v. injection of IR820, IR820-PGMD NPs, and DOX-IR820-PGMD NPs

\section{LIST OF FIGURES}

Figure 1. FTIR spectrum shows the appearance of a $\mathrm{C}=\mathrm{O}$ stretch at $1735 \mathrm{~cm}^{-1}$, typical of ester bonds.

Figure 2. (A) DLS measurement of void PGMD NPs; (B) DLS measurement of IR820-

PGMD NPs; and (C) DLS measurement of DOX-IR820-PGMD NPs.

Figure 3. SEM image of void PGMD NPs.

Figure 4. Temperature curve during 1 hour incubator HT.....

Figure 5. Temperature profile during 3 minutes laser exposure of different concentration of IR820-PGMD NPs.

Figure 6. Cumulative percent release of DOX from DOX-IR820-PGMD NPs under different experimental conditions. .56

Figure 7. Cumulative percent release of IR820 from DOX-IR820-PGMD NPs under different experimental conditions.

Figure 8. 24-hour intracellular DOX uptake in MES-SA and Dx5 cells; $n=3$ experiments,

3 wells per treatment. .58

Figure 9. Subcellular localization of free IR820 and IR820-PGMD NPs in SKOV-3 cells.59

Figure 10. Subcellular localization of IR820-PGMD NPs in SKOV-3. .60

Figure 11. Subcellular localization of DOX-IR820-PGMD NPs in SKOV-3 cells after $24 \mathrm{~h}$ incubation.

Figure 12. 24-hour cytotoxicity profile of void PGMD NPs in MES-SA and Dx5 .62

Figure 13. Cytotoxicity of IR820-PGMD NPs.

Figure 14. 24-hour cytotoxicity profile of NPs and NP free form w/ or w/o 3 minutes laser exposure in MES-SA and Dx5 cells; $n=3,4$ wells/treatment. 
Figure 15. Laser/Dye/NP and incubator HT-induced ROS production were measured in MES-SA and Dx 5 cells.

Figure 16. Laser/Dye/NP and incubator HT-induced HIF-1 expression were measured in MES-SA and Dx 5 cells.

Figure 17. Laser/Dye/NP and incubator HT-induced VEGF expression were measured in MES-SA and Dx5 cells. ..........................................................................68

Figure 18. 24 hours cytotoxicity profile of IR820-PGMD NPs with laser and incubator exposure in MES-SA and Dx5 cells; $n=3,4$ wells/treatment ..............................70

Figure 19. In vivo imaging of free IR820, IR820-PGMD NPs, and DOX-IR820-PGMD NPs.

Figure 20. IR820 plasma concentration at different time points after i.v. injection of free IR 820 or IR820 from NPs. .

Figure 21. DOX plasma concentration at different time points after i.v. injection in DOXIR820-PGMD NP treatment. 


\section{CHAPTER 1: INTRODUCTION}

\subsection{The cancer problem and current cancer therapy}

Cancer has become a serious problem for human society. Currently, it is the $1^{\text {st }}$ and $2^{\text {nd }}$ leading cause of death in developed and developing countries, respectively (1). Human cancer involves different and complicated molecular alterations in cells and its causes are very hard to define. Current clinical therapy for cancer includes chemotherapy, radiotherapy, surgery, and less commonly hyperthermia (HT) and gene therapy, among others. It has been shown that the combination of two or more therapies together, such as radiotherapy with chemotherapy, radiotherapy with HT, or chemotherapy with HT (chemo-thermotherapy), etc. can achieve better outcomes than do single therapies alone (2). Traditional diagnostic and therapy techniques have largely proven insufficient for successful medical management in cancer patients, since they are limited in specificity to tumor tissue, and too often possess very high toxicity to normal tissues $(3,4)$. Some therapies are referred to as "adjuvants", which are treatments given in addition to the main or primary treatment and are intended as an enhancement to the primary therapy. Although there are many therapy methods available today, new methods using advanced technologies are still required for accurate and precise diagnosis, especially in tumor imaging and earlier detection. New strategies to lower or eliminate the side effects of cancer therapy to normal tissues also need to be developed. Among cancer therapies, chemotherapy has been widely used in clinical treatment since many of the anticancer drugs bind to DNA and impair DNA function in fast proliferating cells. However, most, if not all, of these drugs are limited by the fact that they lack specificity, which may result 
in accumulation in normal tissue and exhibit below-therapeutic concentrations at target tumor tissues (4).

\subsection{Nanotechnology and its application in drug delivery}

The development of specific drug delivery approaches was first proposed in 1906, the socalled "magic bullet" (5). The advantages of specific targeted delivery of chemotherapeutic drugs include reducing toxicity in healthy tissue to minimize side effects and at the same time to maximize the therapeutic outcome in tumors. Thus, the developing of optimal drug carriers to achieve targeted delivery has been a primary focus in drug delivery research over the past few decades. Cancer nanotechnology as an emerging new field is being considered for drug carrier applications. Nanotechnology involves multiple disciplines of chemistry, biology, medicine and engineering and is expected to offer many desired cancer therapeutic outcomes with respects to detection, analysis, and treatment $(6,7)$. Some successful examples include the use of natural and synthetic polymeric, metal, and semiconductor nanoparticles (NPs) (8-10), micelles (11), and liposomes $(12,13)$. There are also several studies on medical applications. Hood et al. used polymeric NPs as gene carriers to target to tumor vasculatures (14) and Harisinghani et al. (15) used superparamagnetic iron oxide NPs to detect prostate cancer. Among these delivery systems, natural and synthetic polymeric microparticles/NPs and liposomal delivery systems are very important strategies for cancer drug delivery. 


\subsubsection{Particle delivery system}

Particles can be used for targeted delivery since they are able to protect the drugs/genes/bio-molecules from degradation and increase plasma circulation time (reduce plasma clearance). They are also capable of controlled release and can be tagged with ligands on the surface providing for specific targeting to corresponding receptors on the target cells.

\subsubsection{Liposomal formulation}

Liposomes are colloidal particles which are prepared by phospholipid molecules from either chemical synthesis or natural sources (16). The idea of using liposomes as drug carriers originated in the 1960 s with the discovery that dry lipid films can form spherical enclosed vesicles after hydration (17). Thirty years after their discovery, investigation of the potential applications of liposomes on drug delivery intensified in the U.S. after the Food and Drug Administration (FDA) approved a series of therapeutics based on liposomes. The good biodegradability and biocompatibility of liposomes encouraged scientists to investigate their potential to reduce toxicity and enhance the efficacy of the drug carried in the liposomes. Currently, there are several liposomes and lipid-based products approved for clinical use in the U.S. (18). The surface charge of liposomes may be negative, neutral, or positive depending on the lipid head group composition, which may influence stability and biodistribution, as well as the uptake by targeted cells. To obtain desired pharmacokinetics of liposome encapsulated drugs for both in vitro and in vivo applications, it is necessary to design small liposomes ( $\sim 100 \mathrm{~nm}$ diameter $)$ to obtain 
longer systemic circulation time and minimize reticuloendothelial system (RES) clearance. Researchers have been working on either passive or active targeting of liposomes, which includes attaching polyethyleneglycol (PEG), monoclonal antibodies (mAbs), growth factors, or other molecules on liposome surfaces (19). It is expected that the systemic resident time of liposomes and specificity to targeted tissues may improve after these surface decorations.

\subsubsection{Nanoparticles delivery systems}

Depending on their size, particles can be divided into 3 different categories: macroparticles $(50-200 \mu \mathrm{m})$; microparticles $(1-50 \mu \mathrm{m})$; NPs $(1-1000 \mathrm{~nm})$. Among these different sizes of particles, NPs are being studied intensively due to their small size and capability to escape RES uptake, to enter capillaries, and their ease of uptake by cells through endocytosis. Researchers have been focused on developing lipid, metallic, and polymeric NPs over the past decade. A plethora of the methods have been reported in the literature for the preparation of a large number of polymeric based NPs (20-23). Broadly, these methods can be divided into two categories. The first one is NPs synthesis from the polymerisation of monomers, where NPs are prepared by polymerisation of monomers or a mixture of monomers in an aqueous-organic phase or at the interphase of emulsion or microemulsion. The second category is synthesis from pre-formed polymers in which NPs are formed by various methods such as salting-out, double emulsion, emulsificationdiffusion and emulsification-solvent evaporation methods (24-27). There are mainly two different ways to obtain polymeric NPs: from naturally derived polymers or using synthetic polymers. 
1.2.1.2.1. Naturally derived polymers include fibrin, collagen, chitosan, gelatin, and starch or cellulose derivatives, among others (28). The advantages of natural polymers are that they have very good biocompatibility that may positively support cell adhesion and function, and most of them are biodegradable and usually show low toxicity. However, naturally derived polymers also have disadvantages, such as potential immunogenicity, lesser control over their mechanical properties, inconsistency between batches, risk of animal virus transmission, and high cost due to limited supply (29).

1.2.1.2.2. Synthetic polymers include polyesters such as poly(lactic acid) (PLA), poly(glycolic acid) and the copolymer of lactic and glycolic acid, i.e. poly(lactide-coglycolide) (PLGA) have been extensively explored. Other common classes of polymers used to encapsulate drugs in colloidal systems include poly(amino acids), poly(orthoester)s, and polyanhydrides etc. (30-33). The advantages of using synthetic biodegradable polymers are: (a) good biocompatibility and minimize the risk of biological pathogens or contaminants. (b) controllable biodegradation and release profiles since the degradation rate can be tailored to meet the requirements from several weeks to several years (e.g. by altering LA/GA ratio in PLGA copolymers). (c) can be designed with chemical functional groups that can induce cell growth. (d) specific synthesis methods can be chosen to obtain compatibility with the specific drugs to be delivered and with high drug loading efficiency. (e) inexpensive and reproducible in large scale production with controlled properties.

Synthetic polymers also have disadvantages: (a) release byproducts which could influence cell growth, for instance, acidic degradation products (e.g. lactic acid and 
glycolic acid) can be released in the case of PLGA; (b) have poor ability to undergo processing and can lose of mechanical properties during degradation; (c) chemical synthesis may include toxic substances.

Among the various polymers that can be used to formulate NPs, PLGA is one of the most important. It is a co-polymer of lactic acid and glycolic acid and has been studied intensively since its approval by the FDA for various medical applications. Currently, there are many studies incorporating therapeutic agents into PLGA NPs and used for gene/drug/biomolecules delivery, including our lab $(20,34,35)$.

PLGA can encapsulate hydrophilic and/or hydrophobic drugs. Hydrophobic species, such as paclitaxel, doxorubicin (DOX), or quercetin, can be protected by PLGA from premature degradation so that they can reach their target sites (36). The degradation of PLGA is usually through hydrolysis of the ester linkages (37). The byproducts after degradation include lactic acid and glycolic acid, which can be naturally removed from the body. Extensive studies of PLGA NPs incorporating anticancer drugs have been reported, including entrapping DOX, paclitaxel, dexamethasone etc. (38-40). The criteria for using NPs in drug delivery includes: (a) particles have to be compatible with drugs and achieve high drug loading efficiency; (b) particles have to be stable and have a controllable drug release profile; (c) biodegradation products have to be safe; (d) synthesis process has to be inexpensive and reproducible. 


\subsubsection{Targeted delivery of NPs}

\subsubsection{Passive targeting}

NPs can efficiently reach tumor sites through the enhanced permeability and retention (EPR) effect arising from the lack of an effective lymphatic drainage system as well as the presence of vascular endothelial growth factor (VEGF) promoting angiogenesis which leads to leaky micro-vessels. The endothelial cells in tumor vasculature have loose interconnections and focal intercellular openings. These breaks in the endothelial cell lining range in size between 100 to $780 \mathrm{~nm}$, and NPs carrying the required therapeutic agent can easily extravasate these openings $(41,42)$, which results in increased accumulation of drugs in tumor sites and lower toxicity in other healthy tissues without these vascular properties. The accumulation of NPs in tumor tissue using leaky vasculature is called passive targeting. Another advantage for using NPs as drug carriers is they are able to overcome multidrug resistance (MDR) by bypassing drug exporter pumps (35). MDR may develop in cancer cells due to the cells overexpressing pglycoprotein (P-gp) which pumps out the drugs and compromises the chemotherapy effect (43).

\subsubsection{Active targeting}

The EPR effect facilitates drug escape from the plasma into the tumor interstitium. More specific targeting to tumor cells to obtain even higher treatment efficacy can be achieved by decorating the surface of NPs with appropriate tagging moieties; for instance, ligands, antibodies, or biomarkers to selectively interact with receptors on the tumor cell 
membrane $(18,44)$. The tagging of ligands on NPs aiming at particular receptors on target tissues is called active targeting. There are many ligands that can be used for this purpose, such as peptides, carbohydrates, and monoclonal antibodies (mAbs). Extensive research has been focused on mAbs-based cancer therapies which have shown success towards targeting cancer $(45,46)$. Initially, mAbs were conjugated directly to the drugs. However, this method was not very successful since it often changed the function and bioactivity of the drug during the conjugation process and affected the pharmacokinetics of the drugs $(47,48)$. Our lab has shown that conjugating human epidermal receptor (HER-2) to DOX-loaded PLGA NPs can increase the cellular uptake and cytotoxicity in the HER-2 receptor overexpressed human ovarian cancer cell line SKOV-3, but not in negative HER-2 cancer cell lines such as human uterine cancer lines MES-SA and MESSA/Dx5 (Dx5) (35). Thus, conjugating specific antibodies on NPs to target tumor cells seems to be a promising approach to specific drug delivery.

\subsubsection{The development of multifunctional NPs}

NPs can possess various functional groups on their surface which allows conjugation to multiple agents for improved diagnostics and chemotherapeutics. The idea of the development of multifunctional NPs as a platform for drug delivery has been emerging in recent years. One important advantage of using NPs as a platform for drug delivery is that they can incorporate an imaging agent for imaging tumors simultaneously with delivering chemotherapeutic drugs. Traditional in vivo imaging probes include positron emission tomography (PET), single photon emission computed tomography (SPECT), gadolinium compounds in magnetic resonance imaging (MRI). In comparison, the recent 
development of nano-range molecular imaging probes, such as targeted quantum dots, near-infrared imaging agent encapsulated NPs and other bioengineering NPs provides several unique features. Their optical and electronic properties could be modified by changing the size of the particle, therapeutic agents (e.g. anticancer drugs) can be incorporated into the nanovehicles, and NPs can preferentially accumulate in tumor sites via the EPR effect.

Optical imaging has high temporal resolution, and the imaging system is usually smaller and inexpensive as compared to the more traditional imaging techniques, such as computed tomography (CT), PET/SPECT, MRI etc. However, its clinical application has been limited by low depth penetration since visible light is highly absorbed and scattered by human tissue. In this respect, near-infrared imaging dyes (wavelength 700-900 nm) are promising agents for in vivo optical imaging because light within those wavelengths has minimal absorption by DNA and water, which improves tissue penetration $(49,50)$. Therefore, among a wide range of optical imaging agents, NIR dyes have been studied extensively for in vivo applications, but not dyes that absorb in the visible range due to the reasons mentioned before. Some of the NIR dyes can not only be used as imaging agents but can also act as heat generators due to their unique photothermal properties. Compounds such as cyanine dyes, phthalocyanine and rhodamine derivatives possess this quality (51-53). One of the most important NIR dyes is indocyanine green (ICG), since it is FDA-approved for some medical applications and has been used extensively clinically with a very good toxicity profile. In the past few decades, it has also been studied for its 
potential use in photodynamic therapy, photothermal therapy and optical imaging (54$56)$.

Hyperthermia (HT) (also called thermal therapy, thermotherapy) is the application of heat (usually $41^{\circ} \mathrm{C} \sim 45^{\circ} \mathrm{C}$ ) to cancer cells. In some cases, much higher temperature are used to induce tumor ablation $\left(60^{\circ} \mathrm{C} \sim 80^{\circ} \mathrm{C}\right.$ for $2 \sim 10$ seconds), which result in irreversible destruction of the tumor cells (57). The mechanism of HT is of artificially elevating the temperature of the tissue with the aim of achieving therapeutic benefits and cancer cell killing by damaging proteins and structures inside cancer cells. HT is frequently used together with chemotherapy or radiotherapy to achieve synergistic effects, which can often result in higher cancer therapy efficacy than radiotherapy or chemotherapy alone. There are many successful examples in either phase II or phase III clinical trials for combining HT with radiotherapy, or HT with chemotherapy (58-62). There are also many other research efforts on NPs application to cancer therapy, one of which is by Park's group (63). In their study, DOX-loaded PLGA-Au H-S NPs were fabricated and they showed that the simultaneous delivery of heat and drugs to tumor sites resulted in improved cytotoxicity to cancer cells, inhibiting tumor growth and ultimately destroying the tumor. Currently, gold NPs are being tested for their potential use in photothermal therapy in phase I human clinical trials (64). In addition to gold NPs, superparamagnetic iron oxide NPs are also under clinical investigation for the application of HT (65-67).

One of the disadvantages of using gold NPs is they are not biodegradable. As mentioned above, ICG has also been studied intensively due to its good biocompatibility, biodegradability, and the ability to produce NIR range fluorescence light and heat after 
excitation by a laser at the appropriate wavelength. One of the challenges for using ICG is its poor stability in aqueous solution and short plasma residence time (68). Scientists have been working on encapsulating ICG into NPs, such as PLGA NPs, to increase its stability and extend its plasma circulation time in vivo $(69,70)$.

\subsection{Novel polymeric NPs poly(glycerol-dodecanoate) (PGD)}

As mentioned before, increasing attention has been paid to synthetic polymers for applications in medicine and surgery over the last decades. Nevertheless, very few polymers have successfully entered into clinical trials. The chemistry involved in the synthesis of functional monomers is most often tedious and complex, whereas the subsequent polymerization is difficult to control. Out of these polymer-based agents, polyester based NPs in particular, also have good shelf life, suitable physicochemical properties, and well-characterized degradation products. However, their applications are potentially limited due to their inherent toxicity. Therefore, there is still a need to explore novel biodegradable polyesters in order to overcome these disadvantages and develop clinically translatable drug delivery vehicles.

Migneco and Huang reported the synthesis of a novel polyester from glycerol and dodecanedioic (DDA) for biomedical applications, which maintains good mechanical and biological properties during degradation (71). This novel polyester poly(glyceroldodecanoate) (PGD) was formed mainly through ester bonds and its degradation is through the hydrolysis mechanism. The biodegradation products glycerol and DDA are 
both biodegradable and biocompatible $(72,73)$. Synthesis of the PGD polymer was by mixing of glycerol and DDA in 1:1 molar ratio and heating at $120^{\circ} \mathrm{C}$ for $4 \sim 5$ days. Cell growth studies revealed that PGD-treated plates initially show slower human fibroblast cell growth compared to controls, but at approximately day 18 cell growth in these plates had reached the same level as for control cell culture plates (71). It seems that this polymer is a good candidate for use in drug delivery thanks to its good biodegradability (mainly through surface erosion), biocompatibility, solubility in polar solvents (e.g. ethanol, acetonitrile), and ease and low cost of synthesis.

\subsection{DOX used as chemotherapeutic agent}

Chemotherapy is very often used as a first line therapy for cancer management. DOX and daunorubicin are anthracycline antibiotics which are both often used in human cancer chemotherapy. The anthracycline effectively kills cancer cells by intercalating to nuclear DNA and inhibiting topoisomerase II, leading to cell death $(74,75)$. However, its use has been limited by toxicity to normal tissues, and because of its lack of tissue or organ specificity. The main disadvantage of DOX is that it induces irreversible cardiotoxicity, presumably by localizing in the mitochondria of cardiomyocytes (76). Additionally, cancer cells can develop multidrug resistance (MDR) through the overexpression of P-gp. This results in anthracycline drugs being pumped out of the cells, with the subsequent reduction in therapeutic effect. Scientists are working on incorporating DOX into NPs to overcome MDR and achieve specific targeting to tumor cells. Several groups have incorporated DOX into PLGA NPs $(38,77,78)$. In our lab, we have successfully encapsulated DOX into PLGA NPs that are able to bypass MDR and obtain higher 
cellular uptake and cytotoxicity in P-gp overexpressing MES-SA/Dx5 cells compared to the free form of DOX (35). We further improved the cytotoxicity and cellular uptake of DOX to SKOV-3 by tagging HER-2 antibody on DOX-loaded PLGA NPs.

\subsection{IR820 used as imaging and photothermal agent}

As mentioned above, indocyanine green (ICG) has been studied intensively since it is an FDA-approved NIR dye. ICG (Ex: 785nm, Em: 830nm) can generate NIR range fluorescence light after being excited by a laser. Compared to visible light, absorption by human tissue is markedly reduced for NIR light, thus making it appropriate to be used for in vivo imaging. In our previous work, we have investigated the commercially available cyanine dye IR820, which could be considered as the alternative to ICG because of similar optical and thermal properties (79). A recent study by Massoti et al. conjugated IR820 to polyethylenimine (PEI) for in vivo imaging and DNA delivery (80).

Our study showed the fluorescence quantum yield of IR820 to be lower than that of ICG. However, IR820 has a non-concentration dependent and more predictable absorption peak as compared to ICG (79). Also, IR820 is more stable than ICG in aqueous solution with degradation half-times about double those of ICG. The ability of IR820 to produce heat after exposure to laser is somewhat less compared to ICG. Our work showed that 5 $\mu \mathrm{M}$ IR820 in cell culture media is able to elevate the temperature from $37^{\circ} \mathrm{C}$ to $42^{\circ} \mathrm{C}$ 
during exposure to $3 \mathrm{~min}$ laser with power as $1,440 \mathrm{~J} / \mathrm{cm}^{2}$, while $5 \mu \mathrm{M}$ ICG can cause temperature change from $37^{\circ} \mathrm{C}$ to $45^{\circ} \mathrm{C}$ (79). Although a difference exists, the temperature increase obtained with the use of IR 820 is within the range needed for selective cancer cell HT, and our study showed that IR 820 can be used to significantly inhibit cancer cell growth upon laser exposure in a manner comparable to ICG. In vivo studies showed that the fluorescent signal from IR820 can be detected for longer periods than that of ICG (79). The fluorescence signal of IR 820 was observed to be stronger than ICG in both mice and rats $24 \mathrm{~h}$ after tail vein injection. Rat and mice organ images and dye content studies in specific organs, measured by homogenizing and extracting the dye, also showed that IR820 localized to liver, lungs and kidney, and demonstrated stronger fluorescent signals than ICG in those organs. In sum, IR820 seems to be a good alternative to ICG because of the characteristics mentioned above.

\subsection{Drug release mechanisms in nanoparticles}

Drug release from NPs depends upon many factors, such as polymer composition and morphology, the size and density of the NPs, as well as the physicochemical properties of the drugs, etc. Generally, the investigation of drug release rate involves: (a) the solubility of incorporated drug; (b) desorption of the surface bound/adsorbed drug; (c) drug diffusion through the polymeric NPs matrix; and (d) polymeric NPs matrix erosion/degradation. Thus, the drug release process is governed by drug solubility, diffusion and biodegradation of the NPs matrix. If the drug weakly binds or adsorbs to the surface of the polymer, it may instantaneously dissolve into the releasing solvent, resulting in an initial "burst" release (81). Diffusion-governed release means that the drug 
is released from the NPs core across the polymer matrix and involves the following steps: 1. penetration of water to NPs; 2. swelling of NPs and 3. drug release from the swollen NPs matrix. Moreover, in vitro release of the drug can be affected by the solvent $\mathrm{pH}$, ambient temperature, and the ionic interaction between drug and polymers $(82,83)$.

Polymer erosion can largely affect drug release from the polymer membrane. Depending upon the different polymer materials and size, degradation can take place through bulk erosion or surface erosion. Either one can result in loss of mass from polymer matrices, swelling of polymers, and changes in polymer morphology and molecular weight, etc. (84). There are many factors which could influence degradation rate, including composition of copolymer (85), autocatalysis with acidic degradation products of the polymer (86), interactions between the polymer and the encapsulated drug (87), etc. Burkersroda et al. (88) developed a theoretical model to determine whether polymer erosion occurs through bulk erosion or surface erosion mechanisms. Their model studied the relationship between the degradation rate constant of polymer functional groups $\lambda$ and water diffusivity $\mathrm{D}$, and investigated a parameter called critical device dimension " $\mathrm{L}_{\text {critical }}$ ". The increase ratio of $\lambda / \mathrm{D}$, or an increase of $\mathrm{L}$ can lead to changes in erosion mechanisms. L represents the chemical structure dimension of a polymer matrix and if the polymer matrix dimension is smaller than $\mathrm{L}_{\text {critical }}$, it will undergo bulk erosion. On the other hand, if the polymer matrix is larger than $\mathrm{L}_{\text {critical }}$, it will undergo surface erosion.

\subsection{Introduction of hypoxia-inducible factor-1 (HIF-1)}


Hypoxia is often present in the center of solid tumors due to poorly formed vasculature resulting in low oxygen supply, which could significantly compromise the effect of chemotherapy and radiotherapy (89-92). It is reported that hypoxia-inducible factor-1 (HIF-1), which includes $\alpha$ and $\beta$ subunits, could circumvent the anticancer drug effect by not only escaping drug-induced apoptosis (93, 94), but also through non-apoptotic mechanisms, such as reducing drug-induced senescence in cancer cells (95). Radiotherapy could also be affected since oxygen can bind to unpaired electrons and free radicals that otherwise would damage DNA $(90,96)$. Therefore, effective radiotherapy depends on an adequate availability of oxygen. The inadequate oxygen supply could also result in cancer cell adaptation and lead to expression of various genes in cancer cells, including VEGF, and pyruvate dehydrogenase kinase 1 (PDK1), which are transcriptionally regulated by HIF-1 $(94,97)$.

Given the importance of HIF-1, studies of the effect of HT on this protein are very relevant for therapeutic HT applications in cancer. Moon et al. reported that HIF-1 can be up-regulated by mild HT and lead to the expression of VEGF both in vitro and in vivo, and the increase of VEGF can lead to the promotion of tumor perfusion and vascularization (98). The detailed molecular mechanism of HT induced HIF-1 expression was described in Moon's paper as follows: HT first activates the ERK pathway and causes an increase of the mRNA expression of NOX1, which promotes NADPH oxidase and generate reactive oxygen species (ROS) to up-regulate HIF-1 $\alpha$ expression. The study of Goyal et al. and Chandel et al. also demonstrated that the level of one type of ROS, hydrogen peroxide $\left(\mathrm{H}_{2} \mathrm{O}_{2}\right)$, was increased in cells after NOX1 transfection (99), 
highlighting the importance of $\mathrm{H}_{2} \mathrm{O}_{2}$ in HIF-1 activation in cells (100). A corroborating report by Katschinski's group also described that heat can stabilize the unphosphorylated form of HIF-1 $\alpha$ and up-regulate HIF-1 $\alpha$ expression (101).

ROS was also reported to play a role in the induction of Heat Shock Factor 1 (HSF1) and mRNA accumulation for HSP70, which belongs to the heat shock protein family (102). HSP70 can minimize the effect of heat on cells during heat exposure by up-regulating thermotolerance. This response would compromise the efficacy of HT $(103,104)$. It has also been reported by Madamanchi's group that $\mathrm{H}_{2} \mathrm{O}_{2}$ can up-regulate HSP70 protein levels through the activation of the HSP70 promoter by binding of signal transducers and activators of transcription (STATs) to the promoters in vascular smooth muscle cells (VSMCs) (105). This group exposed VSMCs to $\mathrm{H}_{2} \mathrm{O}_{2}$ and found that the cytoplasmic janus tyrosine kinase 2 (JAK2)/STAT pathway can up-regulate HSP70 and minimize oxidative stress effects on the cells.

Tang's study investigated the effect of HT on cancer cells in a thermal dose dependent manner (106). His study showed that HSP70 was not activated by ICG-induced rapid heating after exposure to laser, and the thermal protective mechanism to cells was not initiated. The HSP70 activity after rapid, short-term and low thermal dose laser/ICG heating was low as compared to the increase expression of HSP70 under slow, long-term and high thermal dose heat accumulation using a cell culture incubator. ICG has been known to generate singlet oxygens after photoactivation, and Fickweiler et al. suggested that the cell killing effect of ICG after laser irradiation was mainly due to photodynamic effect and not the photothermal effect (51). Others have reported that the phothodynamic 
effect would be dominant at low laser densities and high concentrations of ICG, whereas the photothermal effect would be dominant at higher laser energies (up to $20 \mathrm{~W} / \mathrm{cm}^{2}$ ) and lower ICG concentrations (54). This is consistent with the notion that the activation of HSP70 was minimized in a rapid heating modality as described by Tang et al. (106).

As mentioned before, ROS can activate the expression of HSP70. The low expression of HSP70 during rapid-rate heating could possibly mean the abolishment of ROS generation, or abolishment of ROS-induced expression of HSP70. Most of the papers proposing that HT can up-regulate ROS expression and elevate HIF-1 expression and Pgp expression are based on the usage of slower, long-term HT with high thermal dose. It would be important to investigate if rapid and short-term laser/Dye/NP-induced HT, with a lower thermal dose, could result in ROS generation and trigger overexpression of HIF-1 and VEGF. 


\section{CHAPTER 2: STATEMENTS AND SPECIFIC AIMS}

\subsection{Statement of purpose}

Due to the unique and advantageous properties of PGD described above, it may be good candidates for use as a drug delivery vehicle. However, PGD has a low glass transition temperature $\left(\operatorname{Tg}=\sim 32^{\circ} \mathrm{C}\right)$, which creates challenges in achieving controlled release of encapsulated agents. In addition, PGD is hydrophobic, which is not ideal in nanoparticle formulations for drug delivery purposes. In order to overcome these issues, we modified PGD polymers by adding malic acid and adjusting the ratio of malic acid to DDA during the PGD polymer synthesis process in order to adjust the hydrophilicity/hydrophobicity and have a controllable $\mathrm{Tg}$. The formulation technique does not involve toxic chlorinated solvents, unlike the formulation of PLGA NPs, and the characteristics of PGMD NPs can be modified by modulating polymer composition, which provides versatility similar to that of PLGA. Specifically, the glass transition temperature $(T g)$ and hydrophilicity of PGMD NPs can be adjusted by changing the ratio of malic acid to DDA during the PGMD polymer synthesis process. These properties of PGMD polymer make it a good candidate for drug delivery applications. With the controllable $T g$, we could easily manage the release profile, and the PGMD NPs can be used for incorporation of hydrophobic drugs or protein/DNA by adjusting PGMD polymer hydrophilicity. In

addition, PGMD has natural byproducts, such as glycerol, malic acid and DDA, meaning that it is biocompatible and biodegradable. Following the synthesis of PGMD polymers, PGMD NPs were also successfully developed. The synthesis of PGMD NPs is easy and reproducible, and we can prepare uniform PGMD NPs with particles size between 100 to 
$150 \mathrm{~nm}$ for efficient drug delivery. Our goal is to use these PGMD NPs to incorporate the anticancer agent DOX and the imaging and photothermal agent IR820 for delivery to tumors.

Therefore, the purpose of this study was to explore the efficacy of IR820-PGMD NPs and DOX-IR820-PGMD NPs for cancer therapy with in vitro and in vivo assessment, and lay the foundations for future development of targeted delivery approaches. IR820-PGMD NP and DOX-IR820-PGMD NP cytotoxicity in cancer cells were first studied and compared to free IR820 or free DOX plus free IR820 in MDR cancer cell lines (Dx5) and non-MDR cancer cell lines (MES-SA). Our previous studies showed that a higher cancer cell killing effect can be obtained if HT is applied with chemotherapy than by chemotherapy alone (55). However, in that study drug-loaded NPs were not used to deliver the HT. Based on those results, it is expected that both IR820-PGMD NPs and DOX-IR820-PGMD NPs would cause significant cancer cell killing after exposure to laser compared to the no-laser treated group due to the application of HT. Moreover, both NP formulations should be more toxic than free IR820, or free IR820 plus DOX) treatment in Dx5 cells due to NPs can overcome P-gp effect and result in higher cellular uptake and cytotoxicity to Dx5 than unencapsulated DOX and IR820. In addition, these particles also have the potential for targeting.

The expression of ROS, HIF-1 and VEGF after exposure to a faster and shorter-term heating modality with lower thermal dose (laser/Dye/NP HT) were studied and compared to a slower and longer-term heating system with higher thermal dose (incubator HT). In the end, the biodistribution and pharmacokinetics studies were performed using both NP 
formulations and the results were compared to free IR820 and DOX. This basic understanding can serve as a foundation for the ultimate achievement of targeted chemotherapy-HT NPs mediated cancer therapy.

Most researchers have used Au or ICG as near-infrared imaging and HT agent for highlylocalized HT. Our group was the first to explore the potential use of IR820 or IR820 conjugates as HT agent $(79,107,108)$. Several groups, including ours, also analyzed the potential use of IR820 for imaging $(79,80,109)$. To our knowledge, this is the first study to combine DOX and IR820 together and analyze their toxicity to cancer cells and the potential of IR820 as an imaging/HT agent after laser irradiation in newly developed PGMD NPs.

In this study, we investigated multifunctional NP applications in cancer therapy and measured the cellular response after laser/Dye/NP-induced HT and incubator-induced HT. The slow but longer-term heating, as discussed before, would induce production of ROS and activate expression of HIF-1 and VEGF. Tumor angiogenesis occurs partly through activation of expression of VEGF, which is partially regulated by HIF-1. Additionally, HIF-1 is partially responsible for tumor reoxygenation and decreased tumor oxygen consumption, which would be important for radiotherapy. Thus, I believe the study of ROS, HIF-1, and VEGF expression after laser-induced HT is very important in order to explore cancer cell responses in fast-induced HT versus more traditional more slowly-induced HT. In this respect, this research is novel and important being the first to test the laser/Dye/NP and DOX-laser/Dye/NP cancer cell killing/growth inhibition effect 
in vitro with and without laser exposure, and then to analyze the laser-induced rapid heating effect on ROS, HIF-1 and VEGF expression.

For this purpose, in vitro cell culture experiments were conducted on the MDR human uterine sarcoma cancer cell line Dx5 and its non-MDR parent cell line MES-SA. Since the purpose is to establish the efficacy of the proposed treatment modality on cancer cells, normal cell lines are not included.

In the end, we are also interested in performing in vivo pharmacokinetics and biodistribution studies. The purpose of in vivo studies is to compliment the in vitro studies, which provide valuable but limited information since the in vitro model cannot predict and represent the complexity of a living system. Animal models are often used to study a molecular or agent based on the positive in vitro experimental results. We want to explore the potential application of IR820-PGMD NPs/DOX-IR820-PGMD NPs for in vivo optical imaging, and identify the difference between nanoformulations and free drug/dye to see if the NPs can protect the encapsulated agents and result in different biodistribution and pharmacokinetics result from free drug/dye. In the present study the in vivo cancer therapy effects of the NP formulations were not tested however.

Therefore, it is hypothesized that the PGMD NPs will be thermal and pH sensitive, thus a control release of DOX and IR820 will be achieved. The IR820-PGMD NPs and DOXIR820-PGMD NPs will achieve higher cellular uptake and cytotoxicity in MDR cancer cell lines as compared to free IR820 plus DOX. Also, chemotherapy and HT, when applied together in NP formulations with the HT induced by exposure to laser and NIR 
absorbing dye, will induce higher cytotoxicity to cancer cells than chemotherapy alone in both non-MDR and MDR cell lines. In addition, the study will show that the NP formulation can protect the incorporated agents (DOX and IR820) and prolong their plasma half-lives, thus widening the diagnostic and therapeutic window. The slow and longer-term incubator HT, even at a higher thermal dose, does not result in greater cancer cell killing compared to laser HT. Moreover, incubator HT activates the ROS production and results in promotion of HIF-1 and VEGF expression. On the other hand, this study will demonstrate that the combination of HT and chemotherapy in NP delivery form induces a highly-localized and rapid heat accumulation after exposure to laser, with a lower thermal dose, but will not activate ROS production and enhance HIF-1 and VEGF expression, and will result in higher cancer cell killing than in the incubator HT. We believe the study of ROS, HIF-1 and VEGF expression in cancer cells after laser exposure is very important with respect to addressing the effect of different heating rates and thermal doses to cancer cells. We used incubator-induced HT to mimic whole-body HT, and the comparison between incubator HT and laser/Dye/NP HT may provide important information on the effects of different modalities of HT used in cancer therapy. In the end, I believe this study will provide an extension to the current knowledge of thermo-chemotherapy delivery in NP form, and will bring significant impact to the application of nanotechnology on cancer therapy. 


\subsection{Specific aims}

Aim 1, incorporation of IR820 and DOX into void PGMD NPs and characterization of IR820-PGMD NPs and DOX-IR820-PGMD NPs, including Fourier Transform InfraRed (FTIR) spectroscopy, Scanning Electron Microscopy (SEM), Dynamic Light Scattering (DLS) measurements, drug loading efficiency and release kinetics studies in different $\mathrm{pH}$ buffer and with exposing NPs to laser. Cell culture experiments were conducted for studying IR820-PGMD NPs and DOX-IR820-PGMD NPs in both MES-SA and Dx5, including cellular uptake of NPs, NP subcellular localization, NP intracellular fate in the cytoplasm and their cytotoxicity.

Aim 2, determine the production of ROS after laser/Dye/NP-induced HT and compare the results to incubator-induced HT. Then, HIF-1 and VEGF expression were investigated following ROS study. To accomplish this aim, IR820-PGMD NPs were used in an established laser induced rapid heating system to investigate the heating rate and thermal dose effect to the expression of ROS, HIF-1 and VEGF within cells. These results were compared to an incubator HT system, which is used to mimic the traditional whole body HT. The thermal dose of laser/Dye/NP HT and incubator HT was calculated by a cumulative equivalent minutes at 43 degree Celsius $\left(\mathrm{CEM}_{43}\right)$ mathematical model.

Aim 3, pharmacokinetics and biodistribution studies were done in mice. Mouse model was chosen due to their low cost and ease of handling. In addition, the mouse model had been well established for preclinical drug testing to determine their pharmacokinetic behavior and safety before use in humans. Therefore, mice were used to study the 
biodistribution and pharmacokinetics of IR820-PGMD NPs/DOX-IR820-PGMD NPs, and the results compared to free IR820 and free DOX. 


\section{CHAPTER 3: METHODOLOGY}

\subsection{The fulfillment of Specific Aim 1}

3.1.1. Preparation and characterization of PGMD polymer, void PGMD NPs, IR820PGMD NPs and DOX-IR820- PGMD NPs

Doxorubicin hydrochloride (DOX-HCl; MW 579.95) was purchased from Waterstone Technology (Waterstone Technology, CA). Malic acid, 1, 12-Dodecanedioic acid (DDA), dimethylsulfoxide (DMSO $>99.9 \%$, reagent grade), pluronic F-127, micro bicinchonic acid (BCA) protein assay kits, Dulbecco's phosphate-buffered saline (DPBS), phosphate buffered saline (PBS), IR820, penicillin-streptomycin solution, tetrahydrofuran (THF) and trypsin-EDTA were purchased from Sigma-Aldrich (Sigma, Louis). PGMD polymers were prepared following Mingueo's paper with the modification of adding malic acid. Briefly, glycerol (MP Biomedical, LLC, Solon OH) and a combination of DDA and malic acid in 1:1 molar ratio was mixed and heated up to $120^{\circ} \mathrm{C}$ for 48 hours. The molar ratio of DDA to malic acid was 7:3. After that, the mixture was placed under vacuum and continuous stirring for 24 hours. The synthesis of void PGMD NPs, IR820-PGMD NPs and DOX-IR820-PGMD NPs were performed using the oil-in-water emulsification solvent evaporation method. Briefly, approximately $2 \mathrm{mg}$ DOX-HCL and $2 \mathrm{mg}$ IR820 were measured and added to $400 \mu \mathrm{L}$ methanol, then mixed with $0.8 \mathrm{~mL}$ acetonitrile containing around $12 \mathrm{mg}$ PGMD polymer. The mixed solution was added to $12 \mathrm{~mL} 0.1 \%$ pluronic while stirring at $930 \mathrm{rpm}$. The organic phase was removed by evaporation for 24 hours at room temperature. The NPs were then collected and centrifuged at $5000 \mathrm{rpm}$ for 5 minutes to remove large NPs. 
Next, the supernatant was collected and any free DOX or IR820 was filtered out using a dialysis membrane at MWCO 1,000 Da. The dialysis process was continued for 24 hours. Finally, the particles were freeze-dried and lyophilized for 48 hours. Void PGMD NPs were prepared following a similar method except for the addition of DOX and IR820. IR820-PGMD NPs were also prepared following the same protocol, except that no DOX was added.

3.1.2. Characterization of PGMD polymer, void PGMD NPs, IR820-PGMD NPs and DOX-IR820-PGMD NPs

\subsubsection{Characterization of PGMD polymer}

Glass transitional temperature ( $T g$ ) of PGMD was measured through differential scanning calorimetry (DSC) by using a DSC 2910 (TA instrument, New Castle, DE). Fourier transform infra-red (FTIR) spectra were obtained through a Perkin Elmer Spectrum 1000 spectrometer (Perkin Elmer, Waltham, MA). The molecular weight (MW) of the polymer was determined by a calibration curve of standard polystyrene (TSKstandard polystyrene, TOSOH, Japan) measured with a gel permeation chromatography (GPC) column (Jordi lab, Bellingham, MA) at $0.5 \mathrm{~mL} / \mathrm{min}$ flow rate in THF.

\subsubsection{NPs size, size distribution and zeta potential}

Void PGMD NPs, IR820-PGMD NPs and DOX-IR820-PGMD NPs size were measured by dynamic light scattering (DLS) using a Malvern Zetasizer (Malvern Instruments, Worcestershire, United Kingdom). Size measurements were taken at $25^{\circ} \mathrm{C}$ using a 1:30 ( $\mathrm{vol} / \mathrm{vol}$ ) dilution of the NPs suspension in deionized (DI) water. The polydispersity 
index (PDI) was used as a measure of particle size distribution. Zeta potential (surface charge) of the NPs dispersed in DI water was measured by the same Zetasizer. The size of void PGMD NPs was confirmed with SEM (SEM, JEOL-JEM).

\subsubsection{Drug loading}

The NPs were dissolved in DMSO $(1 \mathrm{~mL})$, and the absorption spectrum of the samples was evaluated using a Cary spectrophotometer (Varian, Palo Alto, CA). Serial dilutions of the sample was done to reach the linear range (1:2, 1:4, 1:8, 1:16, 1:32, 1:64 dilutions). The maximum peak intensities were corrected with DMSO blank subtraction and were plotted and fitted to a linear model. Then, the concentration of DOX and IR820 in the NPs were determined using a standard calibration curve of DOX and IR820 in DMSO using the same spectrophotometer. IR820 concentration in IR820-PGMD NPs was measured following the same method using a calibration curve of IR820 in DMSO.

\subsubsection{NIR laser-IR 820 HT delivery system}

\subsubsection{System set-up}

In order to deliver rapid HT to the cells, IR820 has to be first taken up by cells and then activated by a NIR laser. For this purpose, a laser heating system was designed (55). The system includes a laser module (RLDH808-1200-5, Roithner Laserthchnik, Austria), a laser holder, a heated stage insert (WPI Heated Stage Insert, World Precision Instruments Inc, Sarasota) and a mobile stage with an extension arm. External cover of the system is 
also designed for safety consideration; power supplies for the laser and the heated stage insert are also placed outside the box for the same reason.

During operation, the whole system is covered within a wooden box to eliminate effects of air currents and also to prevent the laser light from exiting. The laser module is fixed to a holder, so that its beam is perpendicular to the 96 -well plate which is placed on the heated stage insert. The heated stage insert is powered by an external source to ensure that the plate is at $37^{\circ} \mathrm{C}$ prior to the laser application.

The NIR laser source emits light at $808 \mathrm{~nm}$ with an output power of 1.2 Watts. The calculated power density is $1440 \mathrm{~J} / \mathrm{cm}^{2}$. This small spot size guarantees that only one well is excited at a time. The exact positioning of a well with the laser beam is achieved by moving the stage with an extension arm. The arm is used to move the wells in the plate, thereby allowing different wells to be exposed to the laser one at a time without opening the box.

To verify the positioning of the laser relative to the wells, an infrared beam finder card (IRC-42R) is used to visualize the NIR laser beam while wearing protective goggles. At the same time, the specific well under exposure and its relative position outside the box is indicated by a position indicator on another $96-$ well plate. After moving through A1 to H12 of a 96-well plate using the moving stage, the relative position outside the box is recorded for future use. Necessary safety measures are taken and personal protective equipment used during experiments, especially appropriate engineering and administrative controls. 


\subsubsection{System calibration}

Temperature calibration studies were carried out with the NIR laser system. The purpose is to find the optimal settings for the heated stage insert controller so that a well filled with $222 \mu \mathrm{L}$ of media could be maintained at $37^{\circ} \mathrm{C}$. The temperature distribution across the entire 96-well plate when using the heated stage insert is monitored. During this calibration process, a 96-well plate filled with $222 \mu \mathrm{L}$ of media is placed on the heated stage insert and the settings on the heated stage controller adjusted so that the temperature in the wells is at $37^{\circ} \mathrm{C}$. Twenty minutes time interval is given for steady state heat transfer. Finally, the temperature of each well is recorded with a thermocouple, which is inserted into a 96-well plate to measure the medium temperature change to generate a temperature distribution profile. The temperature distribution of the 96-well plate is shown in Table $1(55)$.

$\begin{array}{llllllllllll}1 & 2 & 3 & 4 & 5 & 6 & 7 & 8 & 9 & 10 & 11 & 12\end{array}$

$\begin{array}{llllllllllllll}\text { A } & 29.3 & 33 & 35.8 & 36.1 & \mathbf{3 6 . 8} & 35.9 & \mathbf{3 6 . 9} & \mathbf{3 6 . 9} & \mathbf{3 6 . 8} & 35.7 & 33.2 & 29.8 \\ \text { B } & 30.2 & 34.4 & \mathbf{3 6 . 8} & \mathbf{3 6 . 7} & \mathbf{3 7 . 1} & \mathbf{3 6 . 8} & \mathbf{3 6 . 5} & \mathbf{3 6 . 9} & \mathbf{3 6 . 6} & \mathbf{3 6 . 8} & 34.8 & 32.7 \\ & & & & & & & & & & & & \\ \text { C } & 32.5 & 35 & \mathbf{3 7 . 4} & \mathbf{3 7 . 1} & \mathbf{3 7 . 2} & \mathbf{3 7 . 1} & \mathbf{3 7 . 2} & \mathbf{3 7 . 3} & \mathbf{3 7 . 1} & \mathbf{3 7 . 2} & 35.7 & 33.8 \\ \text { D } & 35.2 & 35.6 & \mathbf{3 7 . 5} & \mathbf{3 7 . 4} & \mathbf{3 7} & 36.0 & \mathbf{3 6 . 9} & \mathbf{3 7 . 4} & \mathbf{3 7 . 2} & \mathbf{3 7 . 3} & 35.7 & 34.1 \\ \text { E } & 32.3 & 35.5 & \mathbf{3 7 . 6} & \mathbf{3 7 . 2} & \mathbf{3 7 . 1} & 35.1 & \mathbf{3 7 . 4} & \mathbf{3 7 . 1} & \mathbf{3 7 . 3} & \mathbf{3 7 . 1} & 35.9 & 33.9 \\ \text { F } & 33.4 & 35.4 & \mathbf{3 7} & \mathbf{3 7 . 1} & \mathbf{3 7 . 1} & \mathbf{3 6 . 9} & \mathbf{3 7 . 1} & \mathbf{3 7 . 1} & \mathbf{3 7 . 5} & \mathbf{3 7 . 1} & 35.8 & 33.7\end{array}$




\section{$\begin{array}{lllllllllllll}\text { G } & 31.1 & 34.1 & \mathbf{3 6 . 5} & \mathbf{3 6 . 8} & \mathbf{3 6 . 9} & \mathbf{3 6 . 7} & \mathbf{3 7 . 5} & \mathbf{3 7 . 2} & \mathbf{3 7 . 4} & \mathbf{3 6 . 7} & 35.1 & 31.3\end{array}$}

\section{$\begin{array}{lllllllllllll}\mathrm{H} & 29.1 & 33.2 & 35.7 & 35.9 & 36.5 & 36.4 & \mathbf{3 7 . 1} & \mathbf{3 6 . 9} & \mathbf{3 6 . 7} & 35.8 & 34.7 & 29.6\end{array}$}

Table 1. A 96-well plate temperature distribution when placed on the heated stage insert.

Since there is a hole in the center of the heated stage, which results in a nonhomogeneous temperature distribution in the 96-well plate, we designed our experiments accordingly to use the wells which can reach the temperature between $36.5^{\circ} \mathrm{C}-37.5^{\circ} \mathrm{C}$ (bold).

3.1.3.3. Heating capacity of free IR820 and IR820 in IR820-PGMD NPs and DOXIR820-PGMD NPs

We prepared $2.5 \mu \mathrm{M}, 5 \mu \mathrm{M}$, and $10 \mu \mathrm{M}$ solutions of IR 820 or NPs which have equivalent concentration of IR820. Each sample was added to three wells in a 96-well plate sitting on the heated stage insert, and excited with the laser system described in section. 3.1.3.1. for 180 seconds. Temperature inside the well was measured with a thermocouple for the duration of exposure, and the three-well values for a given dye were averaged.

\begin{tabular}{|l|l|l|l|l|l|l|l|l|l|l|l|l|}
\hline & 1 & 2 & 3 & 4 & 5 & 6 & 7 & 8 & 9 & 10 & 11 & 12 \\
\hline A & & & & & & & & & & & & \\
\hline B & & & M & & 2.5 & & 5 & & 10 & & & \\
\hline C & & & & & & & & & & & & \\
\hline D & & & M & & 2.5 & & 5 & & 10 & & & \\
\hline E & & & & & & & & & & & & \\
\hline F & & & M & & 2.5 & & 5 & & 10 & & & \\
\hline
\end{tabular}


Table 2. Schematic of IR820 study design for measuring temperature profile in a 96-well plate (n=3); M: wells with only media; wells labeled with 2.5, 5, 10, indicates the final concentration of IR820 in the well.

The goal of this study was to find out an optimal concentration at which IR820 is able to heat up the cells to approximately $42^{\circ} \mathrm{C}$ quickly. A volume of $22 \mu \mathrm{L}$ of free IR820, IR820-PGMD NPs, or DOX-IR820-PGMD NPs at IR820 concentrations of $25 \mu \mathrm{M}, 50$ $\mu \mathrm{M}$, and $100 \mu \mathrm{M}$ were added to the wells that were originally loaded with $200 \mu \mathrm{L}$ media to achieve final concentrations of $2.5 \mu \mathrm{M}, 5 \mu \mathrm{M}$, and $10 \mu \mathrm{M}$.

\subsubsection{Incubator-induced $\mathrm{HT}$ and temperature profile}

In order to mimic conventional whole body HT, a Hera incubator was used as the energy source. The temperature of the incubator was set to $42^{\circ} \mathrm{C}$. Ninety-six-well plates, which were originally incubated in the $37^{\circ} \mathrm{C}$ incubator, were transferred to the $42^{\circ} \mathrm{C}$ incubator. To study the temperature profile of incubator HT, we first placed the thermocouple inside the incubator and the sensor tip was inserted into a 96-well plate to measure the medium (222 $\mu \mathrm{L})$ temperature change.

\subsubsection{Thermal dose calculation}

The term "thermal isoeffective dose" is used to compare different time-temperature combinations which produce the same cell killing effect. This method was used since the cell death rate under HT is exponentially related to both time and temperature. The 
relationship of time and temperature can be expressed mathematically by the following isoeffect equation:

\section{$t_{1}=t_{2} * R^{T_{1}-T_{2}} \quad$ (Equation 1)}

where $t_{1} \& t_{2}$ are the duration of treatment at temperature $T_{1} \& T_{2}$, respectively. Originally, $\mathrm{R}$ is a function of temperature, however, an estimation of $\mathrm{R}$ as a constant will give an error of less than $2 \%$ in the temperature between $37^{\circ} \mathrm{C}$ to $46^{\circ} \mathrm{C}$ described by Sapareto et al. (110). Hence, $\mathrm{R}$ is assumed to be 0.5 above $42^{\circ} \mathrm{C}$ and 0.25 below $43{ }^{\circ} \mathrm{C}$ (110). Based on Equation (1), different thermal doses can be converted to the cumulative equivalent minutes at $43^{\circ} \mathrm{C}\left(\mathrm{CEM}_{43}\right)$. From Equation (1), we did a slight modification and set $\mathrm{T}_{1}$ to be $42^{\circ} \mathrm{C}$ and $\mathrm{T}_{2}$ to vary during heat treatment, so that we obtain Equation (2). The integral upper limit $t$ is the end time of the experiment ( 3 minutes or 60 minutes).

$C E M_{42}=\int_{0}^{t} R^{42-T(t)} d t \quad$ (Equation 2)

After obtaining the temperature profile during both incubator and laser/Dye/NP HT, the $\mathrm{CEM}_{42}$ model was applied to calculate the thermal doses in each treatment.

3.1.6. In vitro studies of void PGMD NPs, IR820-PGMD NPs and DOX-IR820-PGMD NPs

To fulfill this part of aim 1, human uterine sarcoma MES-SA cells, and their MDR (P-gp overexpressing derivative MES-SA/Dx5 (Dx5)) cells, human ovarian carcinoma cancer cells (SKOV-3), McCoy's 5A medium, and fetal bovine serum were purchased from American Type Culture Collection (Manassas, Virginia). 24-well tissue culture plates, d- 
poly coverslips, and formalin were purchased from Fisher Scientific. All the cells were cultured in McCoy's 5A medium added with 1\% penicillin and 10\% fetal bovine serum, and kept in a $37^{\circ} \mathrm{C}$ cell incubator with a humidified atmosphere of $5 \% \mathrm{CO}_{2}$ and $95 \%$ air.

\subsubsection{IR820 and DOX release kinetics profile from DOX-IR820-PGMD NPs}

Briefly, $5 \mathrm{mg}$ of DOX-IR820-PGMD NPs were resuspended in $3 \mathrm{~mL}$ of $0.01 \mathrm{M}$ PBS $(\mathrm{pH}=7.4$ or $\mathrm{pH}=5.0)$. Next, the sample was divided equally into three Eppendorf tubes, which were shaken at $35 \mathrm{rpm}$ at $37^{\circ} \mathrm{C}$ in an incubator. The tubes were then removed from the incubator every hour up to the first 5 hours, and then after 24 hours. Each time, the samples were centrifuged at $14,000 \mathrm{rpm}$ for 30 minutes. Following this, the supernatant was collected in $4.5-\mathrm{mL}$ cuvettes, and the DOX and IR820 content were estimated using a spectrofluorometer (Jobin Yvon Horiba, Edison, NJ). The NPs were again suspended in fresh PBS solution and incubated for later time release measurements. This process was repeated at regular time intervals, every 7 days after the first day, for a period of 29 days.

The release of DOX from DOX-IR820-PGMD NPs after exposure to laser in different $\mathrm{pH}$ was also studied. Briefly, the NPs were measured and resuspended in $3 \mathrm{~mL}$ PBS with different $\mathrm{pH}(\mathrm{pH}=7.4$ or $\mathrm{pH}=5.0)$ to obtain $5 \mu \mathrm{M}$ IR820. Then, the suspension was exposed to an 808-nm NIR laser (RLDH808-1200-5, Roithner Laserthchnik, Austria) for 3 minutes with power density of $1440 \mathrm{~J} / \mathrm{cm}^{2}$. Finally, the suspension was centrifuged and processed as previously described. 


\subsubsection{IR820 release kinetics profile in IR820-PGMD NPs}

IR820 release kinetics study followed the protocol described in section. 3.1.6.1. IR820 release measurements in different pH PBS were performed only up to 24 hours because the fluorescence intensity of IR 820 becomes unstable after 24 hours. Also, IR820 release was not measured after laser exposure to NPs since photobleaching occurs after laser excitation.

\subsubsection{DOX cellular uptake experiments}

Two cell lines (MES-SA and Dx5) were used to study the cellular uptake of unencapsulated DOX and IR820 (designated as free DOX + IR820) and compare with the uptake of the NP formulation. On the first day, the cells were seeded into 24-well plates at a cell density of 100,000 to 200,000 cells per well. On the second day, the cell medium was removed, and free DOX + IR820 or DOX-IR820-PGMD NPs in growth medium was added to the plates at a normalized DOX concentration of $4 \mu \mathrm{M}(2.3 \mu \mathrm{g} / \mathrm{mL})$. The plates were then placed back in a cell incubator for 24 hours. The control group means no drug was added. After 24 hours, the cell medium was removed, and the cells were washed with ice cold DPBS four times and then lysed with $1 \mathrm{~mL}$ of DMSO. The supernatants were centrifuged at 14,000 rpm for 10 minutes and collected to obtain cell lysates. The DOX fluorescence intensity of cell lysates was measured by a spectrofluorometer (Jobin Yvon Horiba, NJ) at $\lambda \mathrm{ex}=482 \mathrm{~nm}, \lambda \mathrm{em}=590 \mathrm{~nm}$ to determine DOX concentration. To adjust the background fluorescence from cellular components, a DOX calibration curve was created by dissolving DOX and IR820 in DMSO and adding the solution to untreated 
cells. The protein content in the cell lysates can be measured using a micro BCA protein assay kit, and the absorption data was acquired at $562 \mathrm{~nm}$ with the same spectrophotometer. Cellular uptake of DOX for different treatments was calculated by normalizing the DOX amount to the protein amount. An average value was obtained from three wells in each treatment for each experiment, and an average $( \pm$ SD) intracellular uptake of DOX from three experiments was plotted.

\subsubsection{Subcellular localization}

To study the intracellular localization of free IR820, IR820-PGMD NPs and DOXIR820-PGMD NPs, cells were seeded with a density of $4 \times 10^{4}$ cells per well inside wells of a 24-well tissue culture plate, and incubated overnight to reach confluence. On the second day, cell medium was removed and then replaced with $0.5 \mathrm{~mL}$ of $5 \mu \mathrm{M}$ IR820, $0.05 \mathrm{mg} / \mathrm{mL}$ IR820-PGMD NPs (5 $\mu$ M IR820) and $0.05 \mathrm{mg} / \mathrm{mL}$ DOX-IR820-PGMD NPs (4 $\mu \mathrm{M}$ DOX plus $5 \mu \mathrm{M}$ IR820). The plates were kept in a $37^{\circ} \mathrm{C}$ incubator for 24 hours and protected from light exposure. After incubation, cells were washed with PBS three times and fixed with $4 \%(\mathrm{vol} / \mathrm{vol})$ formaldehyde. Then, the specimens were observed by fluorescence microscopy (Olympus IX81, Japan) with a 20X objective or 60X water merged objective. The fluorescence was imaged at $\lambda \operatorname{ex}(490 \mathrm{~nm}), \lambda \mathrm{em}(580 \mathrm{~nm})$ for DOX, $\lambda$ ex $(775 \mathrm{~nm}), \lambda e m(845 \mathrm{~nm})$ for IR820. A CCD camera was used to capture the signals and the images were software-merged with pseudo color (IPLab, Qimaging, Canada). 
For the subcellular localization study of free IR820 and IR820-PGMD NPs. The images were recorded at the same exposure time for both of the treatments in SKOV-3. The acquired fluorescence images were processed by Matlab (MathWorks, Massachusetts) to calculate the image ratio R. First, the intensity of each pixel was background image subtracted, and a region of interest was defined as being composed of any pixels with above-background intensity values (defined as an intensity of at least 2 out of a 255 scale after background subtraction). Background cell images were taken without turning on the fluorescence lamp. The ratio $\mathrm{R}$ was then determined by normalizing the total pixel intensity of this region of interest to its total area.

Lysotracker Blue (Invitrogen, NY) and SKOV-3 were used to identify the subcellular localization of IR820-PGMD NPs with $\lambda$ ex $(355 \mathrm{~nm}), \lambda \mathrm{em}(420 \mathrm{~nm})$. SKOV-3 cancer cells wereused because of their larger size compared to MES-SA and Dx5, which makes it easier to identify the localization of Lysotracker Blue. All the subcellular localization experiments were carried out as described before, with the addition of $5 \mu \mathrm{M}$ Lysotracker Blue to SKOV-3 at the end of the treatment and incubation with cells for 5 minutes.

\subsubsection{Cytotoxicity assessment}

Cell viability was measured with the sulforhodamine B (SRB) assay (Invitrogen, NY), which colorimetrically measures cellular protein. In this study, the cytotoxicity of nine different treatments (laser only group, free IR820, free DOX plus IR820, IR820-PGMD NPs, DOX-IR820-PGMD NPs, IR820 w/ laser, free DOX plus IR820 w/ laser, IR820PGMD NPs w/ laser, DOX-IR820-PGMD NPs w/ laser) were investigated. The detailed 
procedure is the same as we have described in our previous paper (55). Briefly, cells were seeded in a 96-well plate on the first day, and after overnight incubation they were exposed to different treatments. The cytotoxicity was measured with the SRB assay 24 hours post treatment. Tested DOX and IR820 concentrations were at $5 \mu \mathrm{M}$. We also tested the cytotoxicity of void PGMD NPs at higher concentrations $(0.1 \mathrm{mg} / \mathrm{mL})$ than those used in the experiment $(0.05 \mathrm{mg} / \mathrm{mL})$. An average value was obtained from four wells in each treatment for each experiment. Then, an average ( \pm SD) "cell growth" from three experiments was plotted against increasing DOX and IR820 concentrations. Cell growth values were generated by normalizing the data from each treatment to the control values, which did not receive any treatments. Cell growth was calculated by the following formulas: $(\mathrm{Tx}-\mathrm{To}) /(\mathrm{C}-\mathrm{To}) * 100$ if $\mathrm{Tx}>\mathrm{To}$, and $(\mathrm{Tx}-\mathrm{To}) / \mathrm{To} * 100$ if $\mathrm{Tx}<\mathrm{To}$. SRB value To is defined as the initial amount of cells; Tx corresponds to the treatment values; C is SRB value from the controls, which did not receive any treatments. Cell growth was plotted against DOX concentration to show toxicity effects as described by Monks et al. (111) and also used previously by our group (55). If $\mathrm{Tx}>\mathrm{To}$, the treatment is considered as growth inhibition; if $\mathrm{Tx}<\mathrm{To}$, there is no net cell growth after the treatment, so its effect is considered as cell killing. Statistical significance for sample means of cell net growth among treatment groups at the same DOX concentration was identified by one-way analysis of variance (ANOVA). 


\subsection{The fulfillment of Specific Aim 2}

All the assays were carried out in the following groups:

NIR laser-IR820 HT delivery system and incubator HT delivery system, which were described in section 3.1.3. and 3.1.4.

\subsubsection{Study of ROS expression}

Intracellular ROS levels was measured using the fluorescent dye 5-(and-6)-chloromethyl2',7'-dichlorodihydrofluorescein diacetate, acetyl ester (CM-H2DCFDA) (Invitrogen, NY), which was converted into a nonfluorescent derivative (H2DCF) through cellular esterases after uptake by cells. Then, H2DCF can be oxidized to $2^{\prime}, 7^{\prime}$-dichlorofluorescein (DCF), which is highly fluorescent in the presence of ROS. Since this assay requires fluorescence measurements with excitation around $490 \mathrm{~nm}$, which overlaps with the absorption spectrum of DOX, IR820-PGMD NPs were used to measure ROS expression in cancer cells. After HT (either 1-hour incubator HT or 3-minutes NIR-laser induced HT), cells were washed with PBS and collected by incubating briefly with trypsin. The same number of cells were counted and incubated with CM-H2DCFDA at $37^{\circ} \mathrm{C}$ with $5 \%$ $\mathrm{CO} 2$ in the dark. After $30 \mathrm{~min}$, cells were briefly washed with PBS, and the intensity of DCF was measured by flow cytometer (BD Accuri C6, NJ).

\subsubsection{Study of HIF-1 expression}

To investigate HIF-1 expression in both incubator HT and laser/Dye/NP HT, human/mouse Enzyme-Linked ImmunoSorbent Assay (ELISA) was used to detect the 
expression of HIF-1 using HIF-1 $\alpha$ antigen. Basal level HIF-1 expression was identified in cells incubated at normal temperature $\left(37^{\circ} \mathrm{C}\right)$. HIF-1 expression was measured immediately after HT by reading the plate using a fluorescence plate reader (GENios, TECAN, CA) with excitation at $540 \mathrm{~nm}$ and emission at $600 \mathrm{~nm}$ to measure the amount of total HIF-1 in the cells. Then the plate was read with excitation at $360 \mathrm{~nm}$ and emission at $450 \mathrm{~nm}$ to measure the amount of total cytochrome $\mathrm{c}$ in the cells. In the end, the HIF-1 amount was normalized to the amount of cytochrome $\mathrm{c}$ and expressed as HIF-1 activity.

\subsubsection{Study of VEGF expression}

Cancer cell culture medium was collected 6 hours after HT. After centrifuging cell culture media for 10 minutes at $16,000 \times \mathrm{g}, 200 \mu \mathrm{L}$ of supernatant was added into a 96well plate provided in a human quantikine VEGF ELISA kit. VEGF levels were quantified following VEGF ELISA kit protocol and SRB assay was used to determine the amount of cellular protein in each well. Finally, the measured VEGF amount was normalized to SRB value and the calculated results were normalized to controls.

\subsubsection{Cytotoxicity after HT treatment}

Two different heating modalities, including both the incubator and laser/Dye/NP HT delivery system, were used for in vitro Cytotoxicity studies. Detailed descriptions of the heating systems and the temperature calibration for both heating modalities were discussed before in sections 3.1.3. and 3.1.4. Note that when incubator HT was used, 
cells were incubated with the same concentration of IR820-PGMD NPs as used in laser/Dye/NP HT in order to eliminate the effect of NPs by themselves.

\subsection{The fulfillment of Specific Aim 3}

\subsubsection{Mice models}

Thirty-six Nd4 Swiss Webster mice (25-30 grams, 9 weeks old) were purchased from Harlan (Indianapolis, IN), kept under standard housing conditions, and fed ad libitum. All protocols followed the regulations of the Institutional Animal Care and Use Committee. Mice were randomly assigned to different experimental groups based on different time points, namely 15 minutes, 30 minutes, 60 minutes, and 24 hours. On the day of the experiment, the animals were anesthetized and injected through the tail vein with a solution of NPs in PBS. The concentration of injected NPs was determined based on an IR820 dose of $0.24 \mathrm{mg} / \mathrm{kg}$ of body weight and an injection volume of $0.2 \mathrm{~mL}$. The choice of $0.24 \mathrm{mg} / \mathrm{kg}$ was based on the work of Rajagopalan et al., who used a $0.24 \mathrm{mg} / \mathrm{kg}$ dose of ICG to study its pharmacokinetics in mouse blood (112). Our group subsequently also utilized this dose in rat and mice studies of free ICG and free IR820 biodistribution (79). The choice of $0.2 \mathrm{~mL}$ was based on reported safe intravenous (i.v.) injection volumes for mice (113). Therefore, to calculate the amount of free dye/NPs we need to use for animal studies: 
For free dye:

Injection mass $=($ mouse weight in $\mathrm{kg}) *\left(0.24 \frac{\mathrm{mg}}{\mathrm{kg}}\right)$

Free dye solution concentration $=\frac{\text { injection mass }}{0.2 \mathrm{~mL}}$

For NPs, we need to introduce one more term as IR820 loading to $0.24 \mathrm{mg}$ dye $/ \mathrm{kg}$ dose:

Injection mass $=($ mouse weight in $\mathrm{kg}) *\left(0.24 \frac{\mathrm{mg}}{\mathrm{kg}}\right) /\left(\frac{\mathrm{mg} \mathrm{dye}}{\mathrm{mg} \mathrm{NPs}}\right)$

NPs solution concentration $=\frac{\text { injection mass }}{0.2 \mathrm{~mL}}$

Free dye and NPs were first resuspended in DI water or DPBS to reach the final desired concentration and NPs solution was sonicated to make sure they resuspended properly. Then, $0.2 \mathrm{~mL}$ of the solution was drawn into a $1-\mathrm{mL}$ syringe with a $30 \mathrm{~g}$ needle, and the syringe covered with aluminum foil to protect the drug from light. Before the injection, an infrared lamp was used to warm the mouse and facilitate the injection process by dilating the tail veins.

\subsubsection{NIR in vivo biodistribution imaging}

To study the biodistribution, we used an imaging system consisting of a Sanyo DL 7140201S laser (80 mW, $785 \mathrm{~nm})$ and a Retiga CCD camera (Qimaging, Canada) coupled with NIR filter $(\lambda \mathrm{ex}=785 \mathrm{~nm}, \lambda \mathrm{em}=820 \mathrm{~nm})$. The entire setup was covered by BK5 blackout material during the experiment. On the day of the experiment, the mice were first anesthetized by inhalation of isoflurane in a closed chamber, and then injected intraperitoneally (i.p.) with pentobarbital. Animals in the 15-minute group received a 100 $\mathrm{mg} / \mathrm{kg}$ pentobarbital dose. Animals in the 30-minute and 60-minute time groups, received 
the same $100 \mathrm{mg} / \mathrm{kg}$ dose, but administered as two half-doses. The first $50 \mathrm{mg} / \mathrm{kg}$ were given before dye injection and imaging, and the additional $50 \mathrm{mg} / \mathrm{kg}$ were given 15 minutes before the terminal time point in order to reach euthanasia levels. This split administration protocol was used to ensure that the animals would not die before the terminal time point. In the case of the 24-hour group, the animals received a $40 \mathrm{mg} / \mathrm{kg}$ pentobarbital dose on the first day of imaging, were returned to their cage for 24 hours, and received a $100 \mathrm{mg} / \mathrm{kg}$ pentobarbital dose before 24-hour imaging.

For all groups, after the administration of pentobarbital, mice were placed on the imaging stage and a white light image was acquired with white light illumination to determine the position of the mouse. Then, the laser was turned on with white light off to obtain a black image as the background prior to dye injection. Laser current was set to $60 \mathrm{~mA}$ for acquiring optimal fluorescence signal. The exposure time of the images was 10 seconds and threshold gain was 3 . The camera started recording immediately after the injection. A series of images was taken using QCapture Pro software over at least 40 minutes. In the 24-hour group, one white image and one fluorescent image were taken under the same operating conditions 24 hours after the i.v. injection of dye or NPs. After the 24-hour imaging protocol was completed, the animal was euthanized by removal of the heart while under deep anesthesia, and organs were carefully dissected. The lungs, kidneys, liver and intestines were then placed in black-coated Petri dishes and imaged using the same imaging setup. Later, the images were processed with Matlab to calculate the image fluorescence intensity $\mathrm{R}$ as described before in section 3.1.6.4. 


\subsubsection{Pharmacokinetics and biodistribution study}

\subsubsection{Study Design}

Thirty-six healthy Nd4 Swiss Webster mice were divided randomly to different groups with different treatments as shown in Table 3.

\begin{tabular}{|l|l|l|l|l|}
\hline Treatments & 15 minutes & 30 minutes & 60 minutes & 24 hours \\
\hline Free IR820 & Group 1 & Group 4 & Group 7 & Group 10 \\
\hline IR820-PGMD NPs & Group 2 & Group 5 & Group 8 & Group 11 \\
\hline DOX-IR820-PGMD NPs & Group 3 & Group 6 & Group 9 & Group 12 \\
\hline
\end{tabular}

Table 3. Study design for biodistribution experiments of IR820, IR820-PGMD NPS, and DOX-IR820-PGMD NPs. Each group has three mice.

\subsubsection{Pharmacokinetics study}

At the terminal time point for all groups (15min, 30min, 60min, and 24h), plasma samples were collected in order to study the pharmacokinetic profiles of IR820 and DOX after imaging. Plasma samples were obtained by heart puncture followed by centrifugation $2 \mathrm{x}$ for 3 minutes at $12,000 \mathrm{rpm}$. Plasma samples were then incubated in DMSO (1:50 volume ratio plasma: DMSO) for thirty minutes and centrifuged again at $6,000 \mathrm{rpm}$ for 15 minutes. The supernatant was used to perform spectrofluorometric measurements of dye content using previously created calibration curves of IR820 in DMSO at $785 \mathrm{~nm}$ excitation, and DOX in DMSO at $482 \mathrm{~nm}$. The in vivo behavior of the drug/dye can be estimated based on different models, which are based on the assumption that the drug/dye are distributed into one or more so called "compartments", such as organs or body fluids etc. (114). The commonly used model assumes there are input 
(drug/dye administration), mass transfer in compartments, and output (drug clearance) $(115)$.

The one compartment model is the simplest model, which assumes the drug/dye is distributed in the body uniformly and gets eliminated either by metabolism or excretion in urine and feces (115). The equation used to describe the one compartment model is listed below and assumes the molecule follows a monoexponential decay.

\section{$\mathrm{C}(\mathrm{t})=\mathrm{C}_{0} \times \mathrm{e}^{-\mathrm{kt}} \quad$ (Equation 3)}

$\mathrm{C}(\mathrm{t})$ is the concentration as a function of time, $\mathrm{C}_{0}$ is the initial concentration, $\mathrm{k}$ is the clearance rate, and $\mathrm{t}$ is time. Following this equation, the half-life of the molecule, defined as the amount of time it takes for $50 \%$ of the agent to disappear from the system, is calculated by Equation (4):

$\mathrm{t}_{1 / 2}=\frac{\ln (2)}{\mathrm{k}} \quad$ (Equation 4)

A two-compartment model can also be used to estimate the drug/dye in vivo behavior. The assumption of a two-compartment model is that there is central compartment, comprised of the plasma and well-vascularized organs such as the liver; and then the peripheral compartment, which encompasses the rest of the organism. Molecules injected into the system will undergo two distinct processes or phases. In the first phase, distribution, mass transfer occurs from the central compartment to the peripheral compartment until steady state is reached (114). In the subsequent second phase, elimination, the concentration gradient reverses and mass transfer occurs from the peripheral to the central compartment. The mass transfer between peripheral and central compartment will continue during the elimination process until the molecule is cleared 
from the body by liver metabolism and kidney excretion $(115,116)$. Therefore, the equation for a two-compartment model is the bi-exponential decay equation in Equation (5):

\section{$\mathrm{C}(\mathrm{t})=\mathrm{A} \times \mathrm{e}^{-\mathrm{at}}+\mathrm{B} \times \mathrm{e}^{-\mathrm{bt}} \quad$ (Equation 5)}

where $\mathrm{C}(\mathrm{t})$ is the concentration as a function of time; $\mathrm{A}$ and $\mathrm{B}$ are constants for the distribution and elimination process, respectively; a is the rate constant for distribution and $b$ is the rate constant for elimination; $t$ is time (115). Therefore, the model has been separated into two parts: initial rapid decay phase for distribution and second -slow declining phase for elimination. At the starting point $(t=0)$, the sum of $A$ and $B$ is equal to the initial drug/dye concentration.

The distribution process half-life can be calculated as:

\section{$\mathrm{t}_{1 / 2}=\frac{\ln 2}{\mathrm{a}} \quad$ (Equation 6)}

And the elimination process half-life can be calculated as:

\section{$\mathrm{t}_{1 / 2}=\frac{\ln 2}{\mathrm{~b}} \quad$ (Equation 7)}

The choice of the compartmental model is usually based on the a priori prediction of drug/dye in vivo behavior, the goodness of fit between data and model, and the improvement in fit, if any, after using a higher order model.

\subsubsection{Pharmacokinetic analysis of plasma data}

In order to estimate the initial injection dose amount, we first calculated the average weight of all the mice and obtain an average weight of 25 grams. Then, the initial dose 
amount can be calculated as approximately $6 \mu \mathrm{g}$ IR 820 based on the $0.24 \mathrm{mg} / \mathrm{kg}$ injection dosage. The ratio of plasma volume to body weight is $58.5 \mathrm{~mL}$ plasma $/ \mathrm{kg}$ body weight given by the literature (117), so we assumed a 25 -g mouse has plasma volume approximately $1.5 \mathrm{~mL}$. Thus, the initial dose concentration can be calculated to be approximately $4 \mu \mathrm{g}$ dye $/ \mathrm{mL}$ in plasma. Using five data points $(0 \mathrm{~min}, 15 \mathrm{~min}, 30 \mathrm{~min}$, 60min, 24h) and the fit curve toolbox of Sigmaplot (Systat Software, San Jose, CA), we can predict the drug/dye physiology decay behavior with monoexponential or biexponential model. Since there is no study in the literature investigating the IR820 in vivo distribution and elimination behavior, we attempted to generate an in vivo model starting from the simplest model. Moreover, IR820 has similar characteristics as ICG, and ICG pharmacokinetics had been studied via both one-compartment and twocompartment model approaches (118). Hence, we started with the simplest monoexponential model and then the biexponential model to determine which model to be used based on the goodness of the experimental data fit to the models used. For DOX pharmacokinetics, there are also studies using both one-compartment and twocompartment models (119-121), and we also chose the model based on the goodness of fit. As a result, the overall half-life (if one-compartment was used), or the distribution half-life and elimination half-life (if two-compartment was used) can be calculated based on the equations given above. Following the determination of half-lives, we also calculated other pharmacokinetic parameters: the area under the pharmacokinetic curve, the mean plasma residence time, and the clearance rate. 
The area under the curve (AUC) is the integral of drug/dye concentration vs. time curve, which can be calculated by Simpson's rule.

Area under the curve $(\mathrm{AUC})=\int_{0}^{\infty} \mathrm{C}(\mathrm{t}) \mathrm{dt}$ (Equation 8)

The mean residence time in plasma $\left(t_{p}\right)$ is the average time spent by the drug/dye in plasma, and is given by:

$\mathrm{t}_{\mathrm{p}}=\frac{\mathrm{AUC}}{\mathrm{C}_{0}} \quad$ (Equation 9)

where $\mathrm{C}_{0}$ is the model original concentration.

The total body clearance rate (CL) (volume of drug/dye cleared from the body vs. time) is given by:

$\mathrm{CL}=\frac{\text { Dose }}{\mathrm{AUC}} \quad$ (Equation 10)

Based on the preceding calculations, we compared free IR820, IR820-PGMD NPs and DOX-IR820-PGMD NPs pharmacokinetic parameters using one-way ANOVA $(\mathrm{p}<0.05)$.

\subsubsection{Quantitative measurement of organ content}

In addition to plasma measurements, in the $24 \mathrm{~h}$ group the liver, lungs, kidneys, and intestines were also extracted. The quantitative measurements of IR820 content in different organs after 24 hours were performed by dye extraction in DMSO following the procedures described by Saxena et al. for ICG (122) and used by us previously (79). Briefly, the dissected organs (liver, lungs, kidneys, and intestines) were collected and homogenized. Then, the homogenized tissues were incubated with $5 \mathrm{~mL}$ DMSO for at least 4 hours to allow the IR820 and DOX to be dissolved. Then, the supernatant was 
collected and centrifuged at $6,000 \mathrm{rpm}$ for 15 minutes. Next, the fluorescence intensity of the supernatant was measured as described above for measuring plasma in section 3.3.3.2., and the fluorescence intensity was converted to drug/dye concentration in DMSO $(\mu \mathrm{g} / \mathrm{mL})$. Finally, dye mass in $\mu \mathrm{g}$ obtained from this measurement was normalized to homogenized organ mass in grams.

\subsection{Statistical significance}

Statistical significance was identified by ANOVA or t-test (SPSS, Chicago, Illinois) for the difference among treatment groups and control groups. A p-value $<0.05$ was considered to be statistically significant. 


\section{CHAPTER 4: RESULTS}

\subsection{Results accomplished for Specific Aim 1}

\subsubsection{Characterization of PGMD polymer}

The PGMD polymer has a glass transitional $(T g)$ temperature of $42.2^{\circ} \mathrm{C}$, measured by DSC. The $\operatorname{Tg}\left(\sim 42^{\circ} \mathrm{C}\right)$ of PGMD polymer allowed an increased drug release with exposure of the NPs to an external heat stimulus. The MW measured by GPC column is around $3000 \mathrm{Da}$. The Fourier transform infra-red spectra (FTIR) showed an intense $\mathrm{C}=\mathrm{O}$ stretch at $1735 \mathrm{~cm}^{-1}$, indicating the appearance of a typical esteric bond (Figure 1).

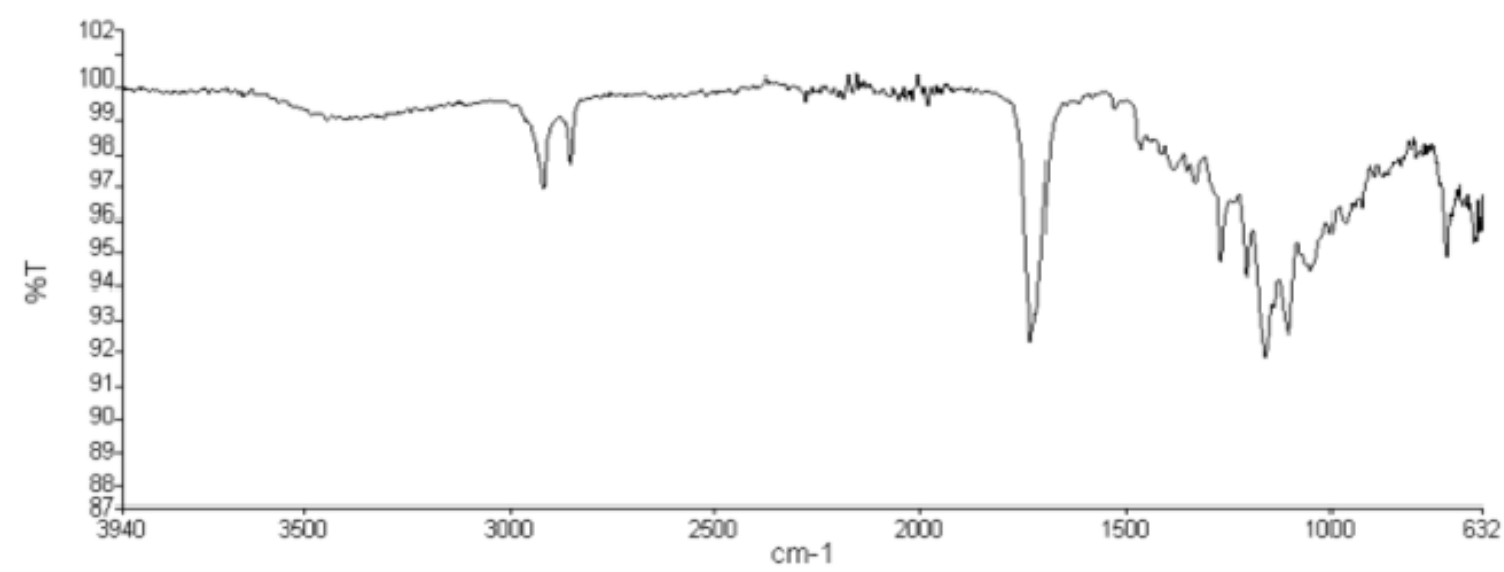

Figure 1. FTIR spectrum shows the appearance of a $C=O$ stretch at $1735 \mathrm{~cm}^{-1}$, typical of ester bonds.

4.1.2. Characterization of void PGMD NPs, IR820-PGMD NPs and DOX-IR820PGMD NPs. 
The size, zeta potential, polydispersity (PDI), and drug loading efficiency for void PGMD NPs, IR820-PGMD NPs and DOX-IR820-PGMD NPs are shown in Table 4. The size and shape of void PGMD NPs are also given in DLS measurement (Figure 2), and SEM (Figure 3).

\begin{tabular}{|l|l|l|l|l|l|}
\hline Formulation & $\begin{array}{l}\text { Size } \\
(\mathrm{nm})\end{array}$ & $\begin{array}{l}\text { Polydispersity } \\
(\mathrm{PDI})\end{array}$ & $\begin{array}{l}\text { Zeta } \\
\text { potential } \\
(\mathrm{mV})\end{array}$ & $\begin{array}{l}\text { IR820 } \\
\text { loading } \\
(\mathrm{w} / \mathrm{w} \%)\end{array}$ & $\begin{array}{l}\text { DOX } \\
\text { loading } \\
(\mathrm{w} / \mathrm{w} \%)\end{array}$ \\
\hline PGMD void NPs & $92 \pm 19.6$ & $0.095 \pm 0.015$ & $-34.3 \pm 1.6$ & $\mathrm{~N} / \mathrm{A}$ & $\mathrm{N} / \mathrm{A}$ \\
\hline $\begin{array}{l}\text { IR820-PGMD } \\
\text { NPs }\end{array}$ & $109 \pm 8.2$ & $0.151 \pm 0.006$ & $-29.1 \pm 7.5$ & $8.4 \pm 0.5$ & $\mathrm{~N} / \mathrm{A}$ \\
\hline $\begin{array}{l}\text { DOX-IR820- } \\
\text { PGMDNPs }\end{array}$ & $125 \pm 19.7$ & $0.182 \pm 0.023$ & $-20.3 \pm 2.9$ & $8.1 \pm 0.6$ & $4.3 \pm 0.3$ \\
\hline
\end{tabular}

Table 4. Mean size, PDI, zeta potential, and percent of loading efficiencies for void PGMD NPs, IR820-PGMD NPs, and DOX-IR820-PGMD NPs $(n=8)$.

The NPs are between 50-200 $\mathrm{nm}$ range, which can potentially avoid premature clearance by the RES.
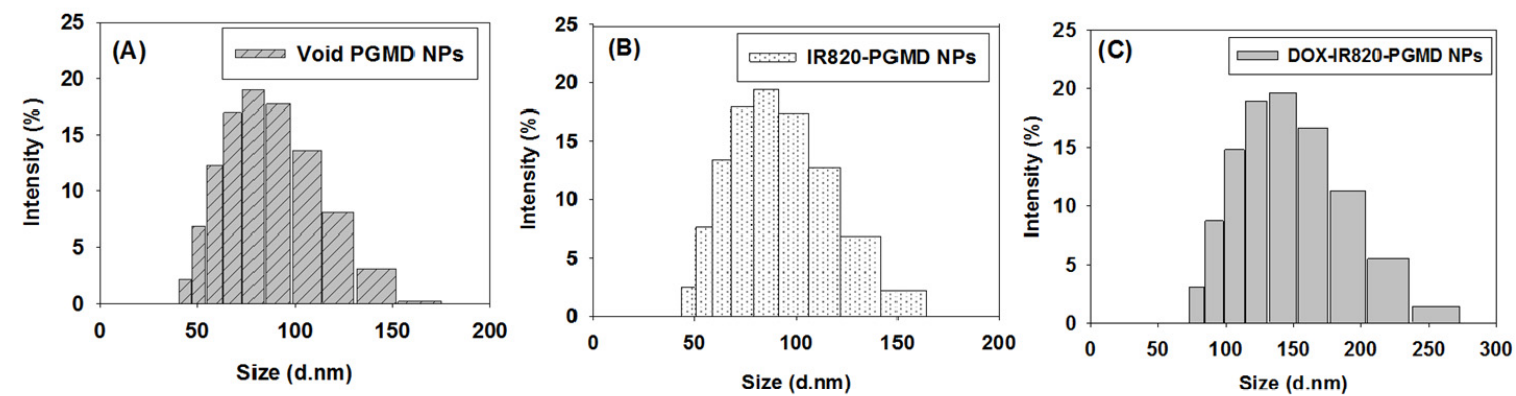
Figure 2. (A) DLS measurement of void PGMD NPs; (B) DLS measurement of IR820PGMD NPs; and (C) DLS measurement of DOX-IR820-PGMD NPs.

The SEM image was used to confirm the size and shape of void PGMD NPs and it showed that void PGMD NPs were nearly spherical and uniformly distributed.

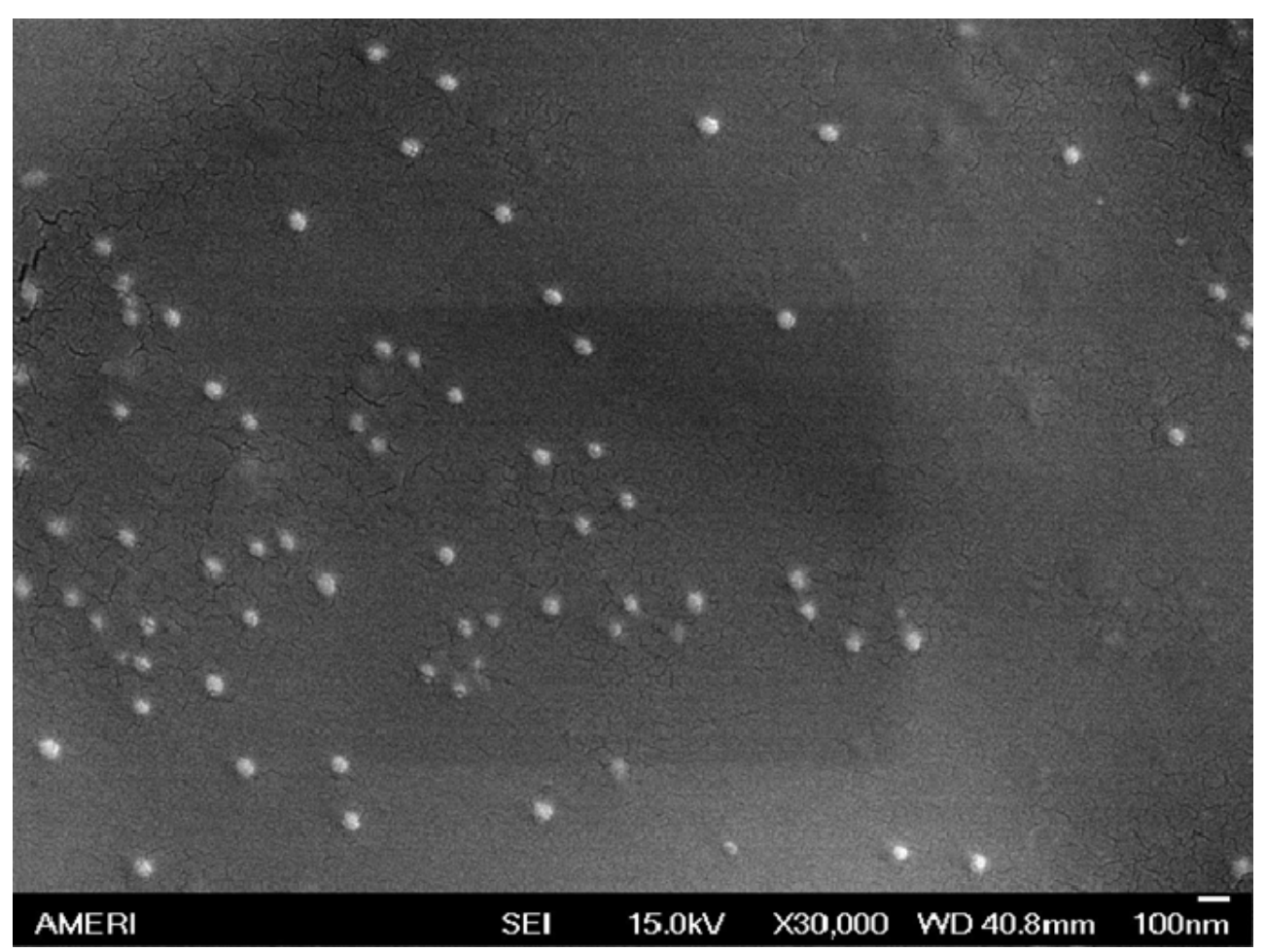

Figure 3. SEM image of void PGMD NPs.

\subsubsection{Heating modalities}

\subsubsection{Incubator HT delivery system and temperature increase profile}

Temperature calibration studies were carried out to find the temperature profile of the fluid inside the wells of 96-well plates as shown in Figure 4. The temperature increased in incubator HT is very slow, taking approximately 45 minutes to reach $42^{\circ} \mathrm{C}$. 


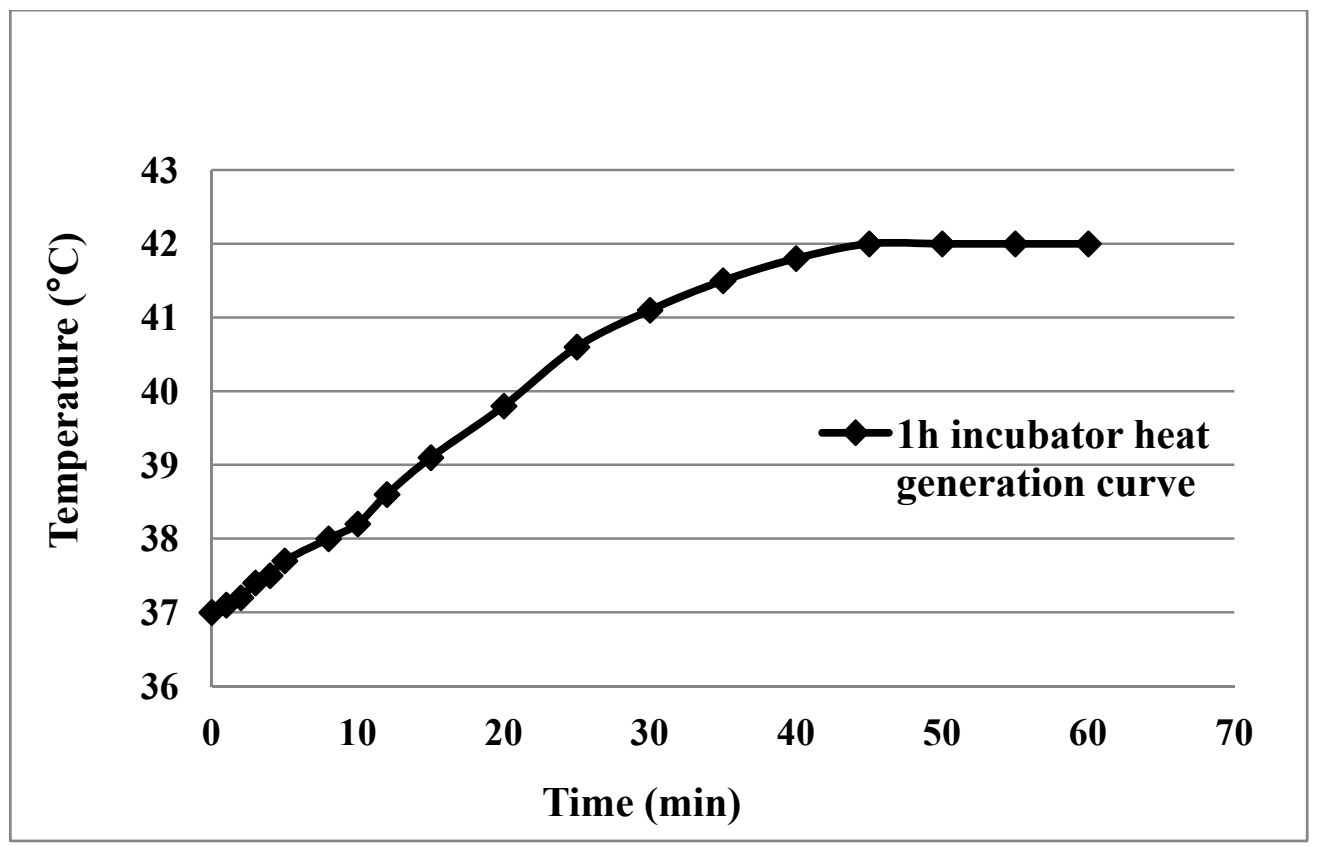

Figure 4. Temperature curve during 1 hour incubator HT.

4.1.3.2. Laser-IR820-PGMD NPs HT delivery system and temperature increase profile

The temperature profile of IR820-PGMD NPs is shown in Figure 5. We used different concentrations of IR820-PGMD NPs in order to find out the optimal concentration which could induce a temperature increase to between $41^{\circ} \mathrm{C} \sim 45^{\circ} \mathrm{C}$. As shown in Figure 5, 0.05 $\mathrm{mg} / \mathrm{mL}$ IR820-PGMD NPs, which has $5 \mu \mathrm{M}$ IR820, can elevate the temperature to approximately $42^{\circ} \mathrm{C}$. Since this temperature is very close to $T g$ of PGMD polymer and it is sufficient to induce HT effect to cancer cells, we chose to perform our studies based on 0.05 mg/mL IR820-PGMD NPs/DOX-IR820-PGMD NPs. 


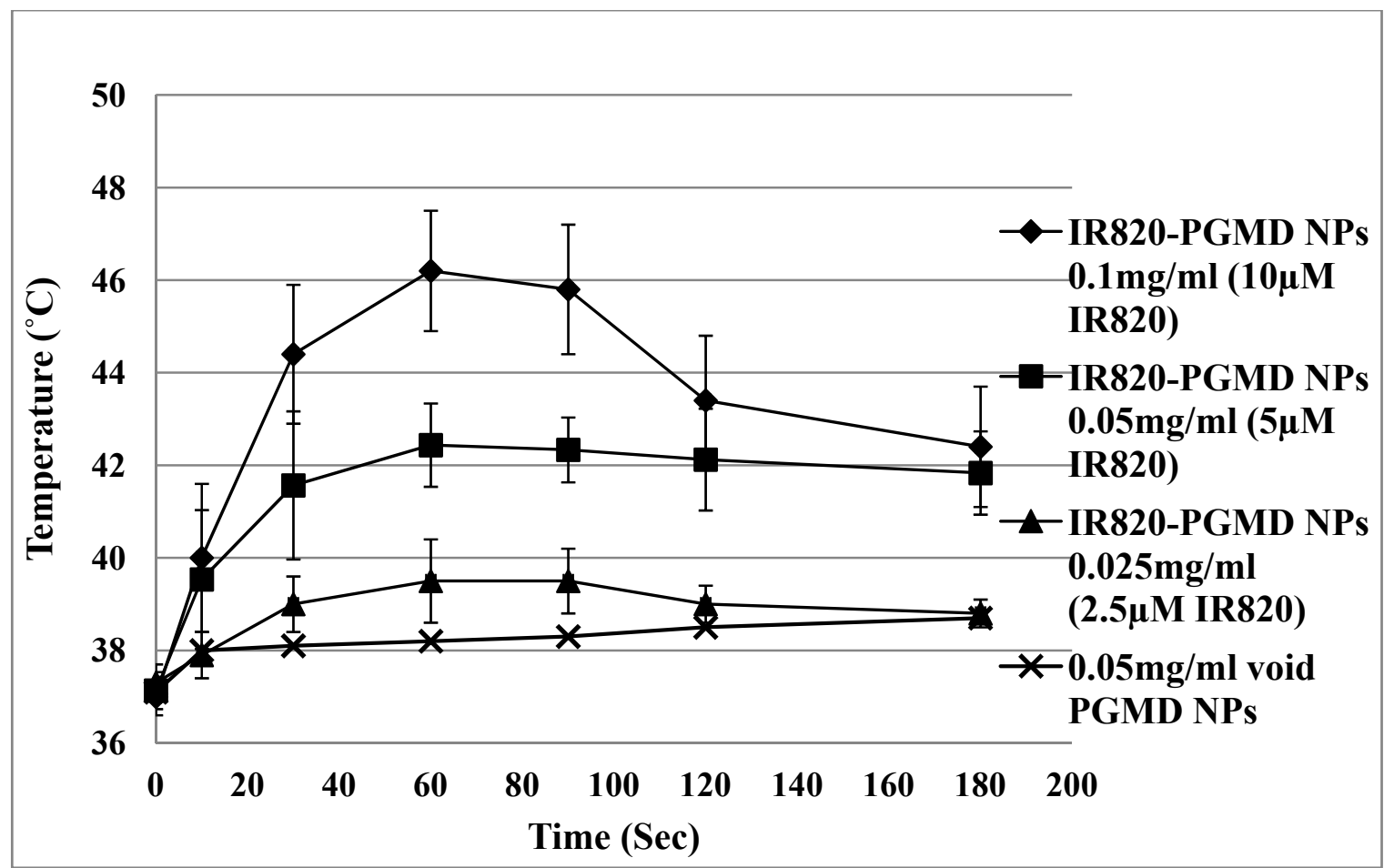

Figure 5. Temperature profile during 3 minutes laser exposure of different concentration of IR820-PGMD NPs. Experiments were repeated 3 times.

\subsubsection{Thermal dose calculation}

A much slower temperature increased was observed in incubator HT compared to the temperature increase in laser/Dye/NP exposure. Thermal doses given in these two treatments were calculated based on the $\mathrm{CEM}_{43}$ model developed by Sapareto et al. (110) as described before. We made a slight modification to use the model at $42^{\circ} \mathrm{C}\left(\mathrm{CEM}_{42}\right)$ with a smaller empirical value $\mathrm{R}=0.25$. As shown in Table 5 , laser/Dye/NP HT for 3 minutes with $5 \mu \mathrm{M}$ IR820-PGMD NPs produced a much lower thermal dose as compared to the $42^{\circ} \mathrm{C}$ incubator HT treatment for 1 hour. 


\begin{tabular}{|c|c|}
\hline Treatment type & $\mathrm{CEM}_{42}(\mathrm{~min})$ \\
\hline $5 \mu \mathrm{M}$ IR820-PGMD NPs $+3 \min$ laser & 3.06 \\
\hline $5 \mu \mathrm{M}$ IR820-PGMD NPs $+42^{\circ} \mathrm{C}$ incubator $1 \mathrm{~h}$ & 25.98 \\
\hline
\end{tabular}

Table 5. Calculated $C E M_{42}$ in different types of HT treatment, based on the temperature curves in Figures 4 and 5 and using Equations 1 and 2.

4.1.5. The in vitro studies of void PGMD NPs, IR820-PGMD NPs and DOX-IR820PGMD NPs

\subsubsection{In vitro drug release}

The release kinetics profile of DOX from DOX-IR820-PGMD NPs is shown in Figure 6. In $\mathrm{pH}$ 7.4 PBS, the burst release of DOX was around $49 \%$ in the first 5 hours, followed by a slow release reaching a total of only $\sim 52 \%$ after 29 days. When these NPs are exposed to the laser for 3 minutes in $\mathrm{pH} 7.4 \mathrm{PBS}$ at the beginning of the experiment, 5 $\mu \mathrm{M}$ IR820 is able to increase temperature to approximately $42^{\circ} \mathrm{C}$, inducing a rapid release of $\sim 81 \%$ DOX in 5 hours and $85 \%$ in 29 days. Additionally, an acidic environment $(\mathrm{pH}=5.0$ ) can also induce the release of DOX from the NPs to up to $\sim 72 \%$ in 5 hours and $86 \%$ in 29 days. DOX release was further enhanced when the NPs were placed in acidic buffer and exposed to laser for 3 minutes. Overall, $90 \%$ DOX was released in 5 hours and $\sim 95 \%$ in 29 days. We observed a very slow release of DOX after the initial burst release from NPs in the absence of an external stimulus. However, either acidic buffer or heat can induce the release of DOX, indicating that PGMD NPs are thermal sensitive and $\mathrm{pH}$ sensitive. 


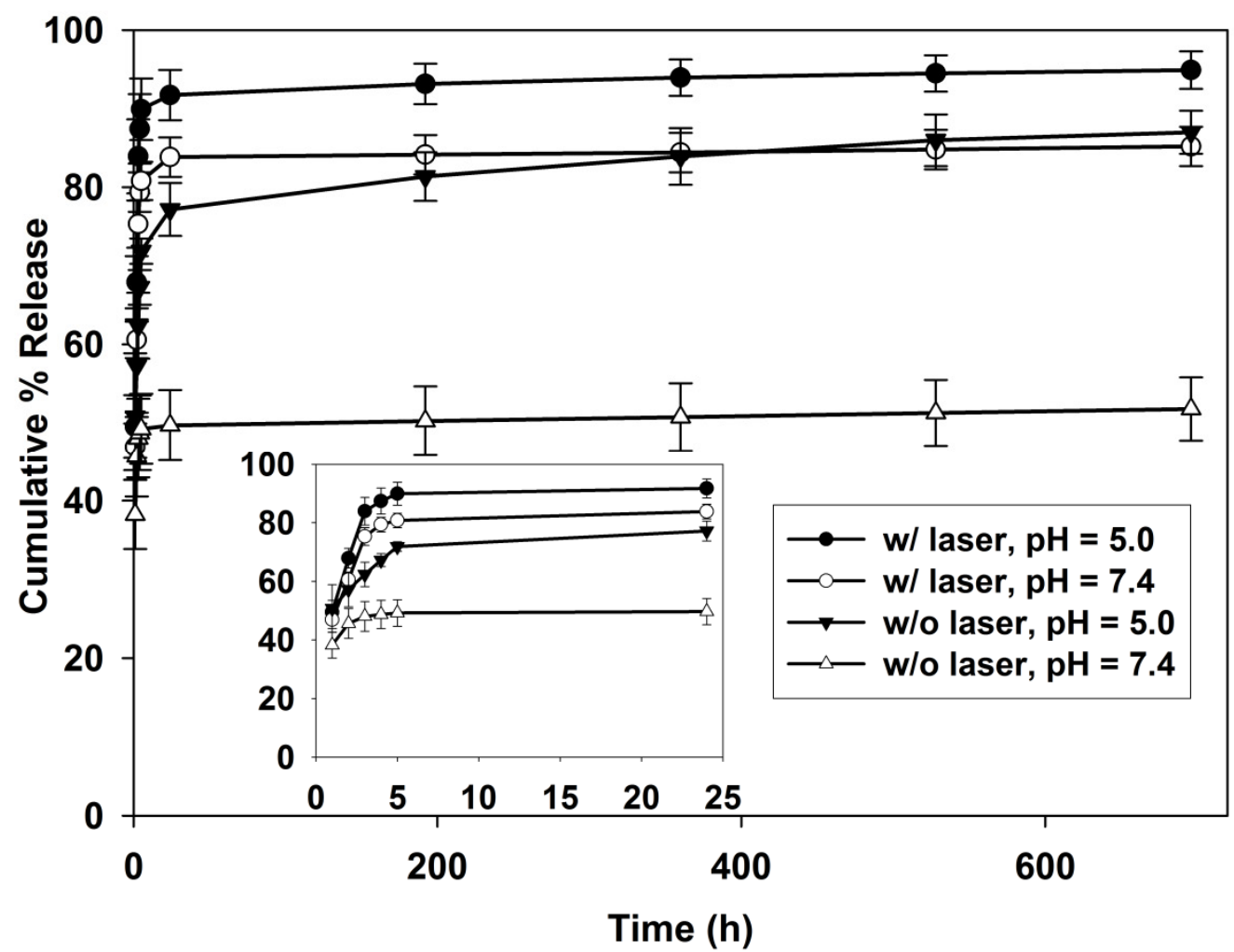

Figure 6. Cumulative percent release of DOX from DOX-IR820-PGMD NPs under different experimental conditions.

The release of IR820 from DOX-IR820-PGMD NPs is shown in Figure 7. IR820 release was also enhanced when NPs were placed in an acidic environment. Approximately $48 \%$ IR820 was released in 5 hours in acidic PBS $(\mathrm{pH}=5.0)$, and up to $52 \%$ IR820 was released in 24 hours. Since the PGMD NPs are $\mathrm{pH}$ sensitive, it was expected that an acidic environment would induce higher amount of IR820 release from NPs as compared to a neutral environment $(\mathrm{pH}=7.4)$. IR820 measurements were performed only up to 24 hours because the fluorescence intensity of IR820 becomes unstable after 24 hours. Also, 
IR820 release was not measured after laser exposure to NPs since photobleaching occurs after laser excitation.

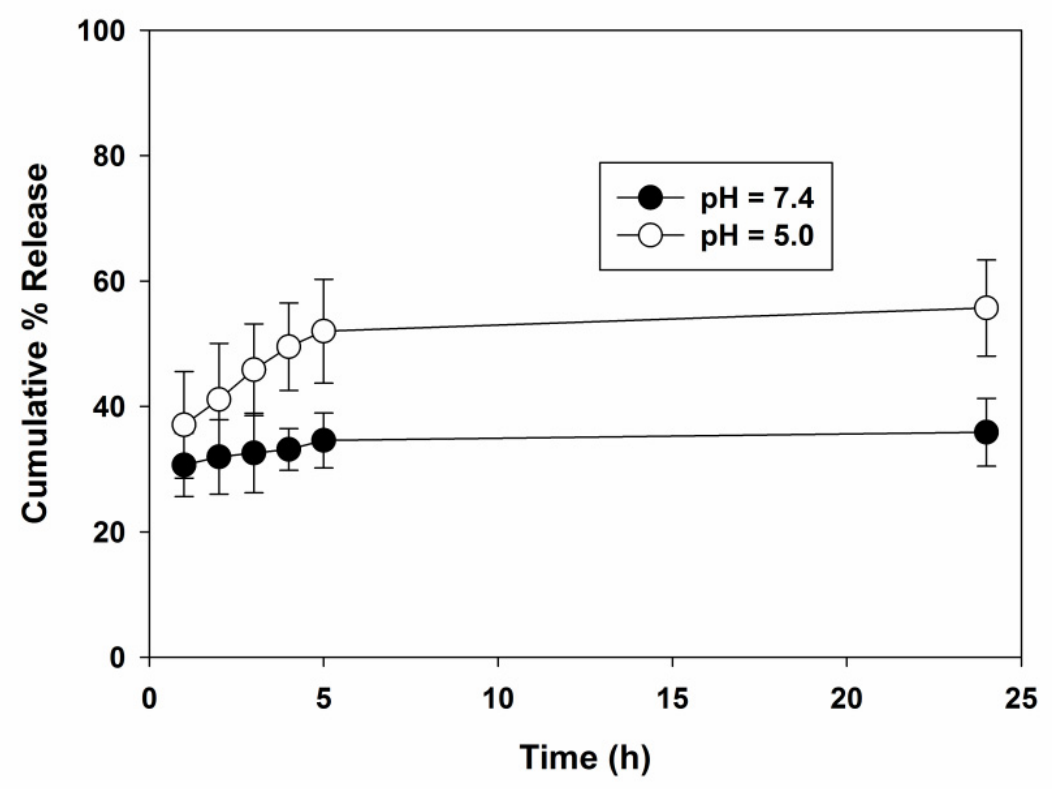

Figure 7. Cumulative percent release of IR820 from DOX-IR820-PGMD NPs under different experimental conditions.

\subsubsection{DOX cellular uptake experiments}

The cellular uptake results are consistent with the literature in that free DOX is taken up by cells mainly through diffusion (123), while the NPs formulation is generally delivered into cells by endocytosis (124). An improved DOX cellular uptake profile by NPs was observed as compared to their free form in the MDR cell line Dx5 as shown in Figure 8, probably due to reduced elimination of drug with NPs delivery since NPs formulation can overcome the P-gp effect. However, NPs did not result in greater DOX uptake in drug- 
sensitive cancer cell line MES-SA compared to the free drug form, since these cells do not have mechanisms to affect drug retention.

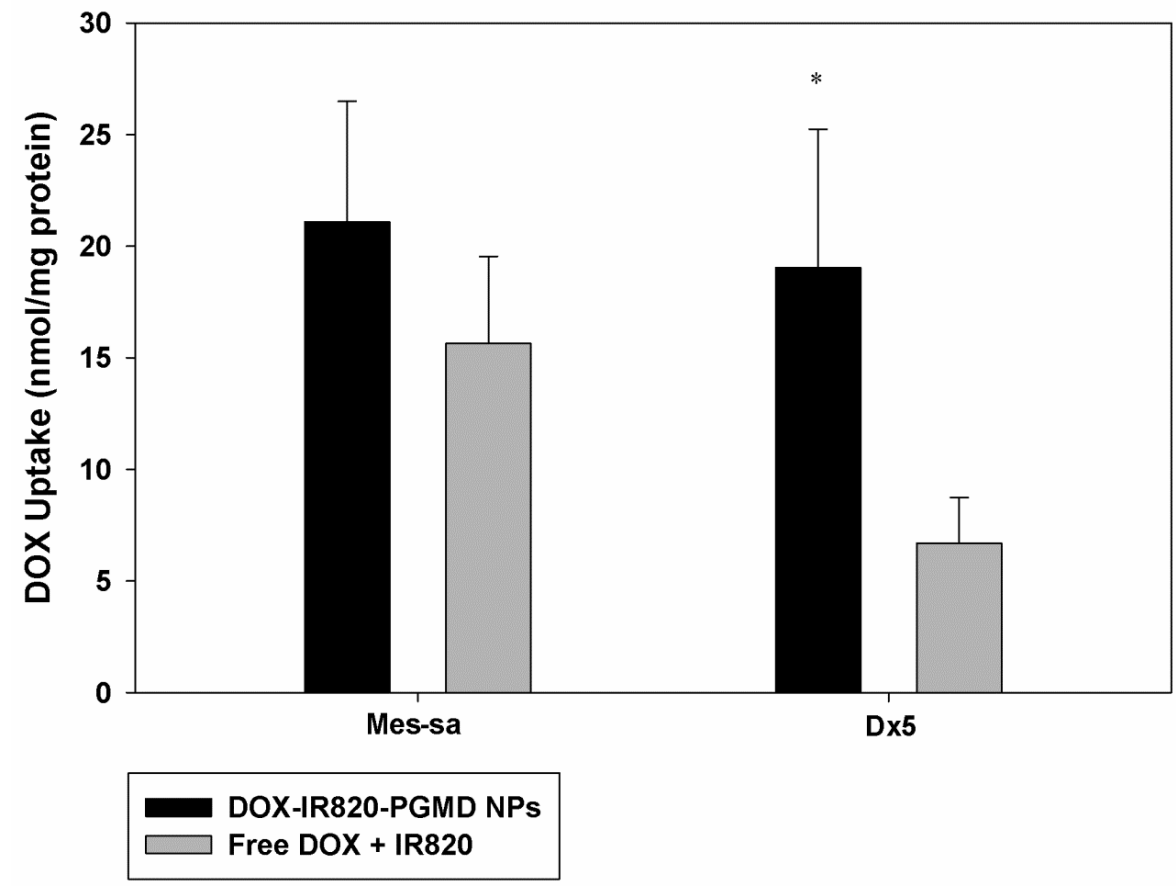

Figure 8. 24-hour intracellular DOX uptake in MES-SA and Dx5 cells; $n=3$ experiments, 3 wells per treatment. $* P<0.05$ (by t-test) between NP formulation and NP free form for each cell line, indicating significant differences due to loading of DOX into PGMD NPs.

\subsubsection{Subcellular localization}

Subcellular localization of $5 \mu \mathrm{M}$ free IR820 and $0.05 \mathrm{mg} / \mathrm{mL}$ IR820-PGMD NPs (equivalent to $5 \mu \mathrm{M}$ IR820) is shown in Figure 9. The subcellular localization of both treatments is similar. Free IR820 possibly binds to cytoplasmic proteins such as ligandin (125), leading to widespread cytoplasmic localization. In the case of the NP formulation, IR820 released from the NPs will behave the same as free IR820, whereas IR820 still 
within the NPs will be located in endosomes/lysosomes. Images from three different experiments of free dye and NPs treatments were chosen for statistical studies for fluorescence intensity $(\mathrm{n}=3)$ and the $\mathrm{R}$ values from the fluorescence microscope images show that the NP formulation produces a higher intracellular fluorescence intensity $(\mathrm{R}=3.75 \pm 0.54)$ than does the free dye $(\mathrm{R}=2.89 \pm 0.23)$ after 24 hours of incubation, although the difference is not statistically significant, possibly due to the small sample size ( $\mathrm{n}=3$ for each group).
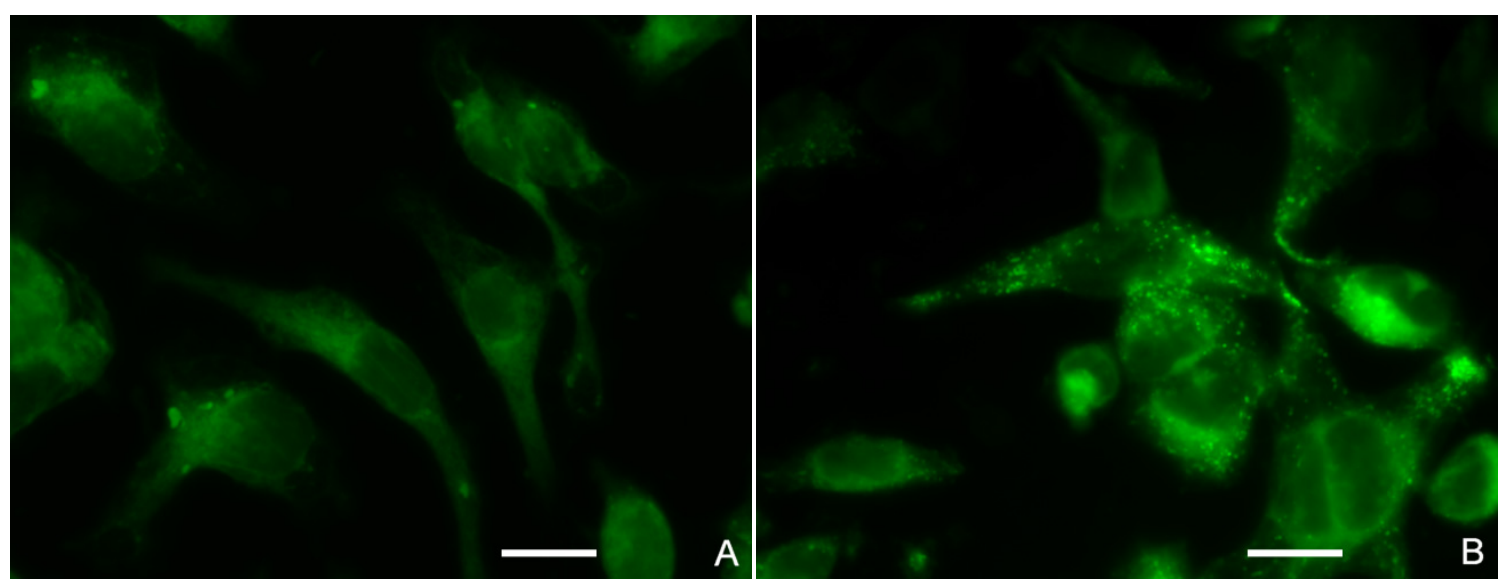

Figure 9. Subcellular localization of free IR820 and IR820-PGMD NPS in SKOV-3 cells. All images were taken with 60X water-merged objective after 24 hours incubation of NPs with cells and merged with pseudo color by software. A. IR820 fluorescence of free IR820; B. IR820 fluorescence of IR820-PGMD NPs. Scale bar represents $20 \mu \mathrm{m}$.

IR820-PGMD NP subcellular localization images are shown in Figure 10. In Figure 10A, Lysotracker Blue was used to stain SKOV-3 lysosomes, while Figure 10B shows the fluorescence of IR820. Figure 10C, which is the merged image, shows that PGMD NPs 
are mainly localized in the lysosomes as indicated by the overlap with the Lysotracker Blue. This result indicated that most PGMD NPs were probably taken up into cells by endocytosis. However, further studies are needed to investigate the specific endocytic pathways used by the cells.
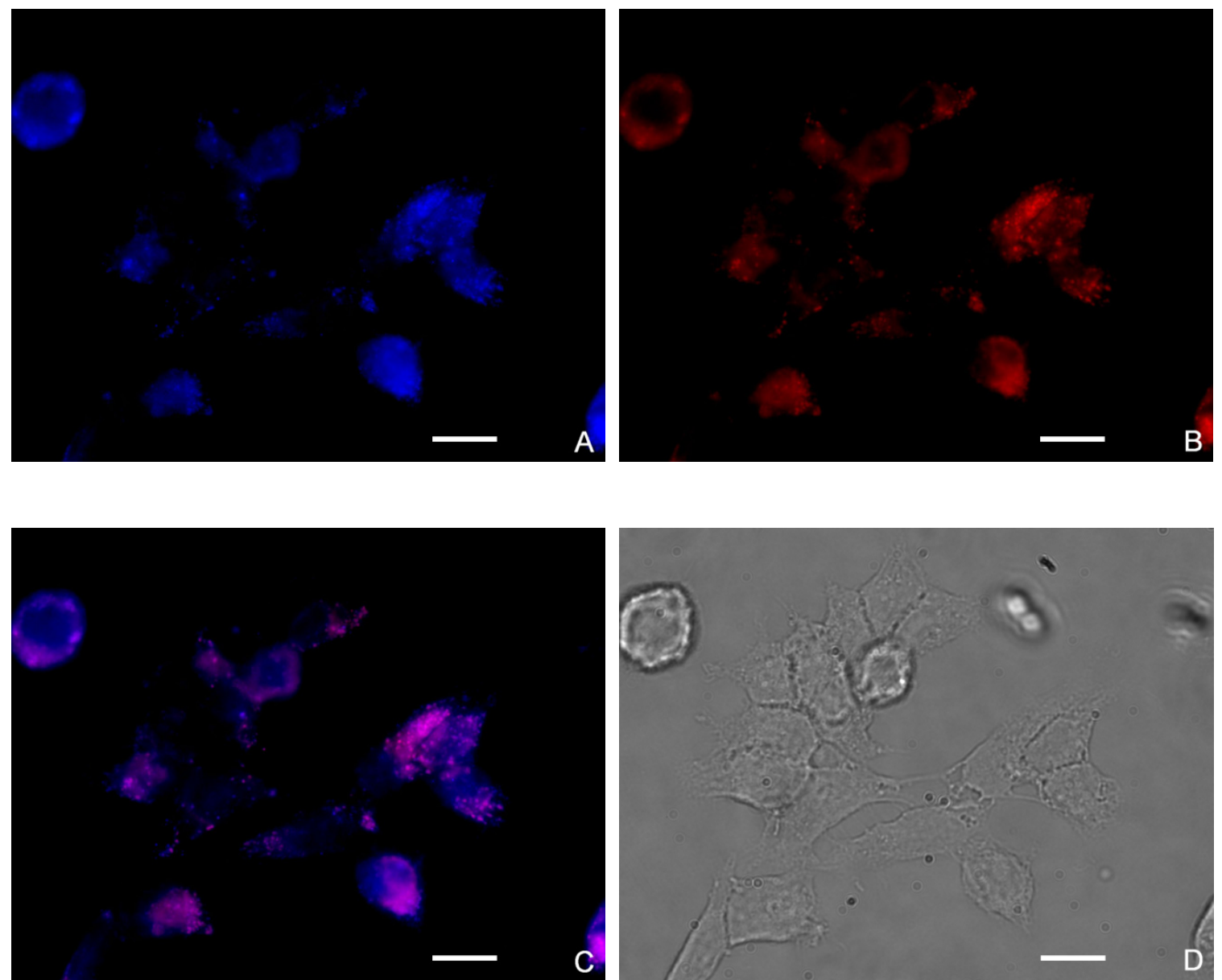

Figure 10. Subcellular localization of IR820-PGMD NPs in SKOV-3. All the images were taken after 24 hours incubation of NPs with cells and merged with pseudo color by software (IPLab, Qimaging). A. Lysotracker Blue fluorescence; B. IR820 fluorescence of IR820-PGMD NPs; C. merged picture of $A$ and B; D. phase contrast image. Scale bar represents $20 \mu \mathrm{m}$. 
As seen in Figure 11A, we observed that some DOX molecules were located in the nucleus. Since the free drug accumulates in the nucleus, we would expect the same fate for DOX leaked out from NPs (74). On the other hand, DOX molecules that remained in the NPs stayed in the cytosol because size limitations prevent the NPs from crossing the nuclear pore complex. In Figure 11B, we can see that IR820 stayed in the cytosol for both the free form and the molecules that were in NP form. Free form localization is due to the possible binding of free IR820 to cytoplasmic proteins such as ligandin (126). In the merged picture shown in Figure 11C, the yellow bright dots in the cytosol indicate that the NPs are still in the process of releasing DOX and IR820.
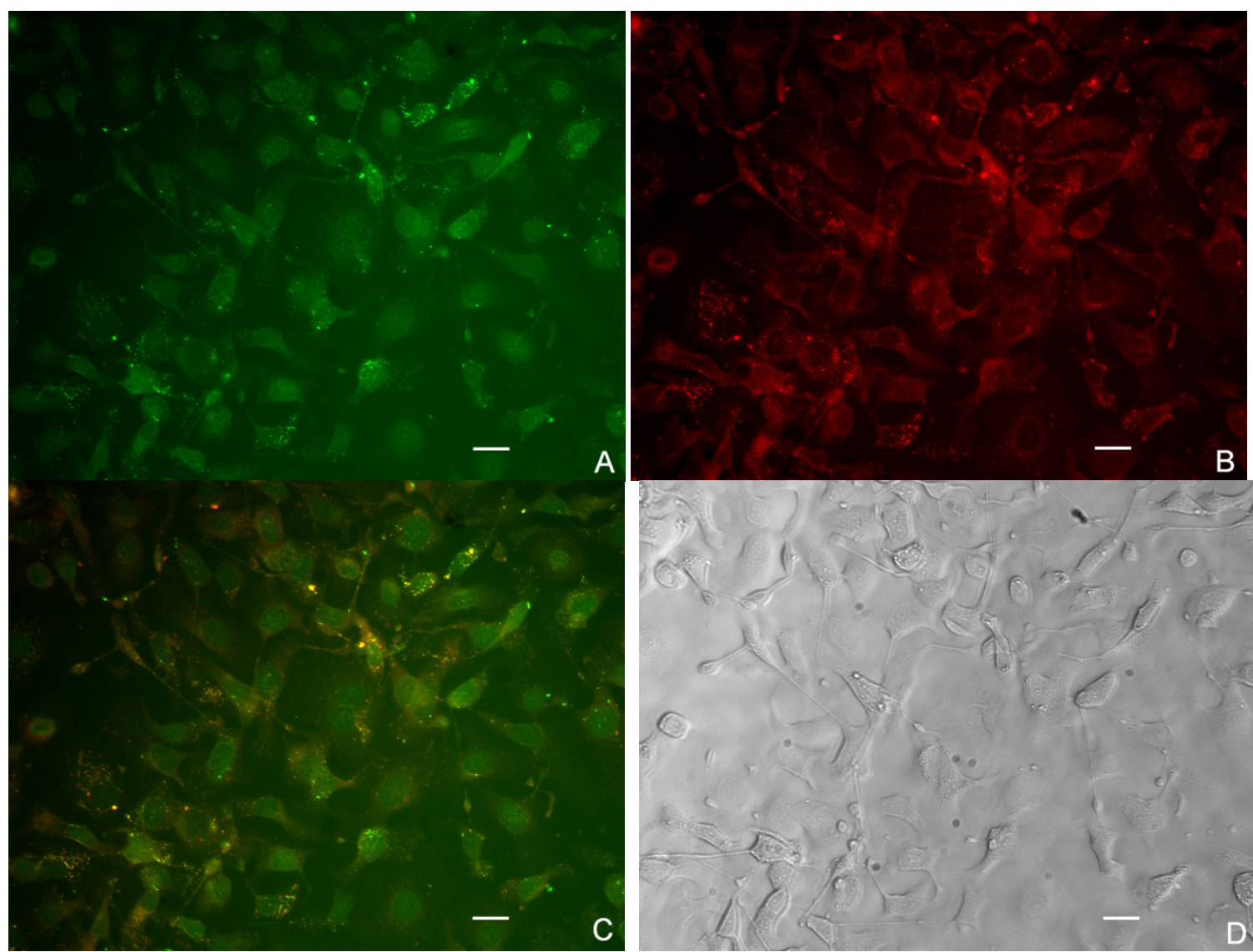
Figure 11. Subcellular localization of DOX-IR820-PGMD NPs in SKOV-3 cells after $24 \mathrm{~h}$ incubation. A. DOX fluorescence of DOX-IR820-PGMD NPs; B. IR820 fluorescence of DOX-IR820-PGMD NPs; C. merged picture of $A$ and B; D. phase contrast image. The concentrations of IR820 and DOX were kept at $5 \mu M$ and $4 \mu M$, respectively. Scale bar represents $20 \mu \mathrm{m}$.

\subsubsection{In vitro cytotoxicity}

\subsection{Void PGMD NPs cytotoxicity profile}

Void PGMD NPs cytotoxicity profile is shown in Figure 12. We tested the NP concentration up to $0.1 \mathrm{mg} / \mathrm{mL}$ in both MES-SA and Dx5 cells and no toxicity was observed in either cell line. The desired NPs concentration $(0.05 \mathrm{mg} / \mathrm{mL})$ is based on the DOX and IR820 loading result, which is in the safe range of using PGMD NPs.

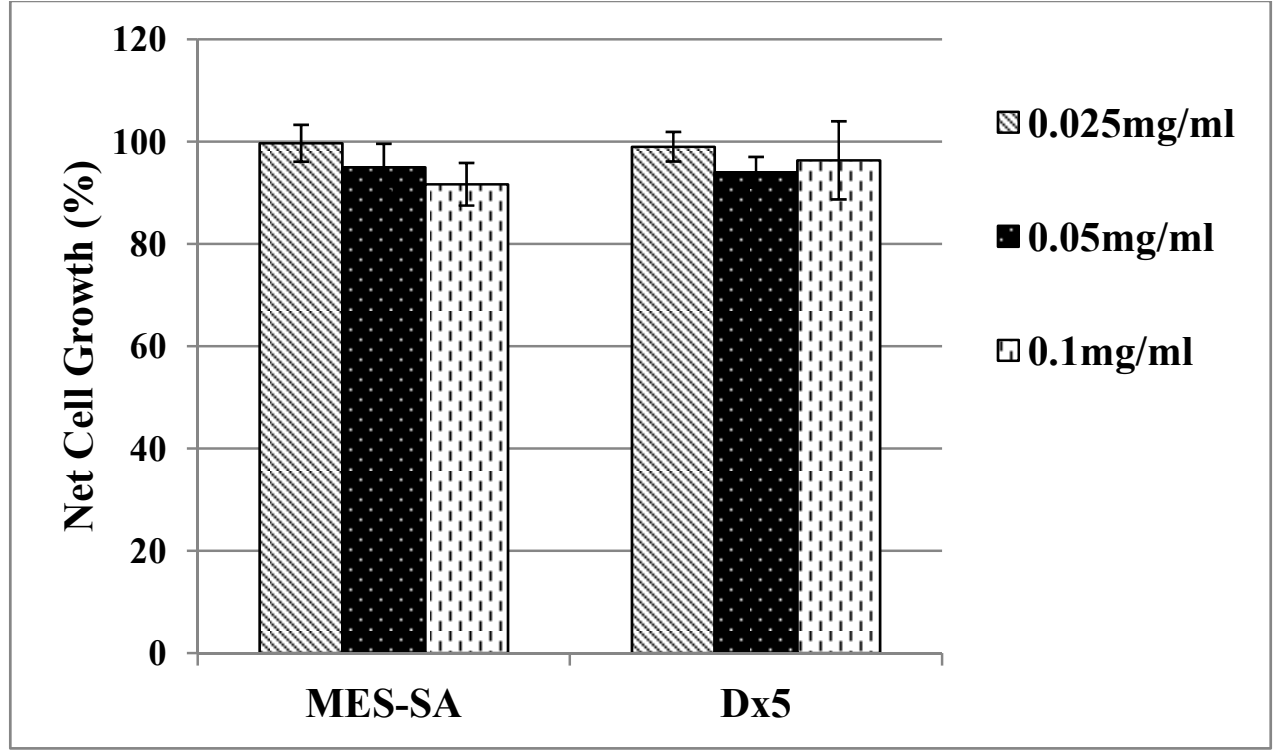

Figure 12. 24-hour cytotoxicity profile of void PGMD NPs in MES-SA and Dx5. 


\subsection{Cytotoxicity of IR820-PGMD NPs}

Figure 13 shows the cytotoxicity of IR820-PGMD NPs in MES-SA and Dx5 in different concentrations. It is shown that IR820-PGMD NPs does not cause any toxicity effect in SKOV-3 and Dx 5 cells at $0.05 \mathrm{mg} / \mathrm{mL}$ (5 $\mu \mathrm{M}$ IR820), whereas it results in approximately $15 \%$ cell growth inhibition in MES-SA. This is probably because the fact that MES-SA cells are more sensitive to environmental stressors than SKOV-3 and Dx5. The same phenomena was observed when $5 \mu \mathrm{M}$ free IR820 was incubated with MES-SA cells in our previous study (79).

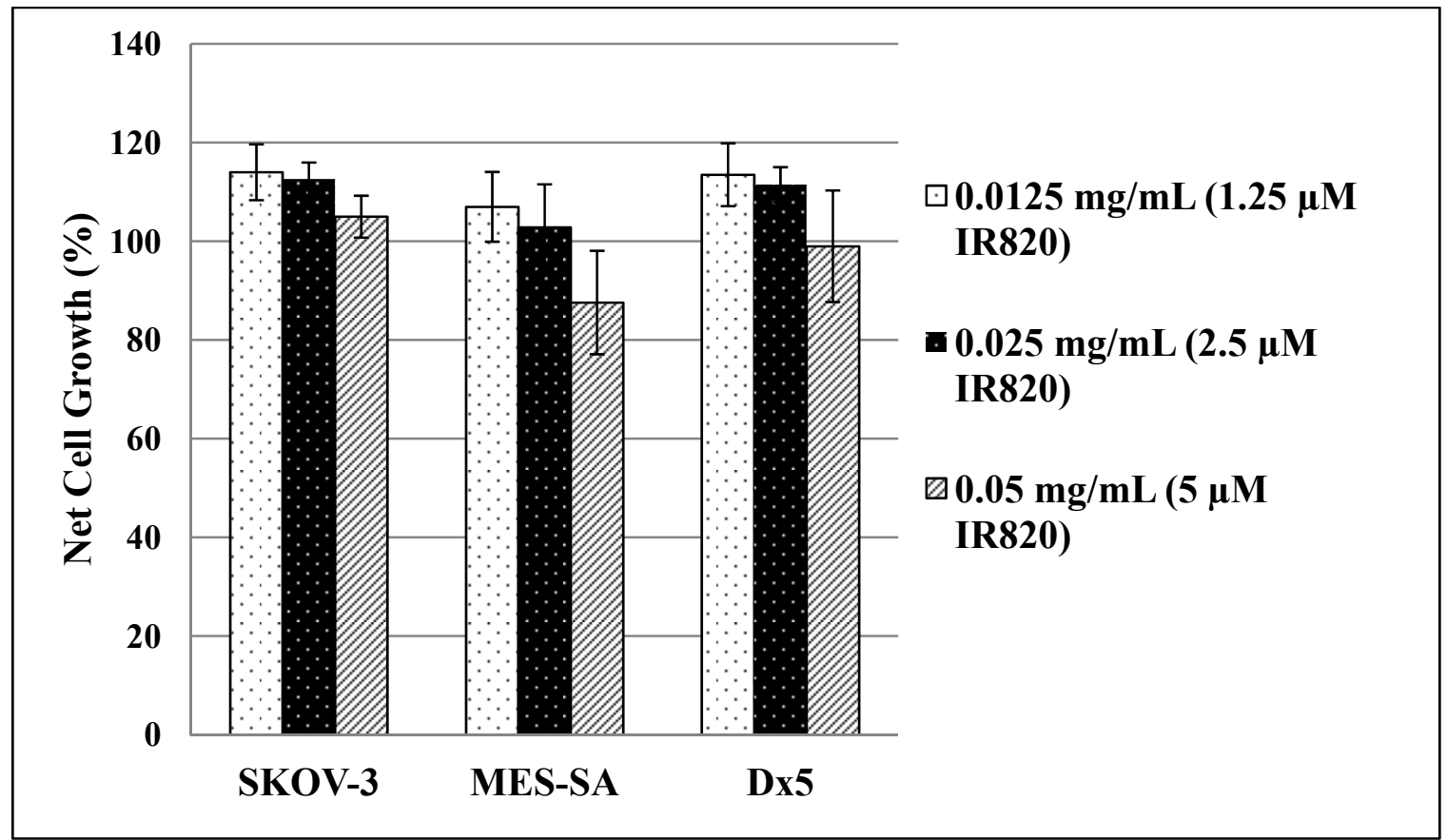

Figure 13. Cytotoxicity of IR820-PGMD NPS. 
4.1.5.4.3. Cytotoxicity of IR820-PGMD NPs, DOX-IR820-PGMD NPs with exposure to laser as compared to free IR820 and free DOX + IR820

MES-SA and Dx5 cell proliferation following IR820-PGMD NPs, free IR820, DOXIR820-PGMD NPs, and free DOX + IR820 incubation w/ or w/o laser exposure is shown in Figure 14. A solution of $0.05 \mathrm{mg} / \mathrm{mL}$ IR820-PGMD NPs or DOX-IR820-PGMD NPs (containing approximately $5 \mu \mathrm{M}$ IR820) can increase the temperature from a baseline of $37^{\circ} \mathrm{C}$ to $42^{\circ} \mathrm{C}$ following exposure to an $808 \mathrm{~nm}$ NIR laser (power density is $1440 \mathrm{~J} / \mathrm{cm}^{2}$ ) for 3 minutes, as shown above. Based on this finding, we used a concentration of 0.05 $\mathrm{mg} / \mathrm{mL}$ IR820-PGMD NPs/DOX-IR820-PGMD NPs in our study and compared them to free IR820/ free DOX + IR820 treatment. Both NP formulations and the free agent treatments produced significant cancer cell killing after laser exposure compared to the no-laser group due to the HT effect. It is important to note that laser treatment by itself does not have an effect on cell growth.

Our results showed that, although IR820-PGMD NPs seems to have higher cytotoxicity than free IR820 after laser exposure in MES-SA, the difference did not reach statistical significance. The same phenomena were observed in DOX-IR820-PGMD NPs compared to free DOX + free IR820 in MES-SA cells without laser exposure, and the difference in cancer cell killing is not statistically significant either. On the other hand, IR820-PGMD NPs and DOX-IR820-PGMD NPs showed much higher cytotoxicity than free IR820 or DOX-IR820 treatment in Dx5, and the difference is statistically significant $(\mathrm{p}<0.05)$. 
Additionally, the combination of HT and chemotherapy caused enhanced cancer cell killing in both cell lines compared to either chemotherapy or HT alone $(\mathrm{p}<0.05)$. Based on our results, the treatment of the resistant Dx 5 cells with NPs containing $4 \mu \mathrm{M}$ DOX and 3 minutes of laser exposure can improve the cytotoxicity and the cell killing effect to reach levels comparable to those observed in DOX-sensitive MES-SA cells.

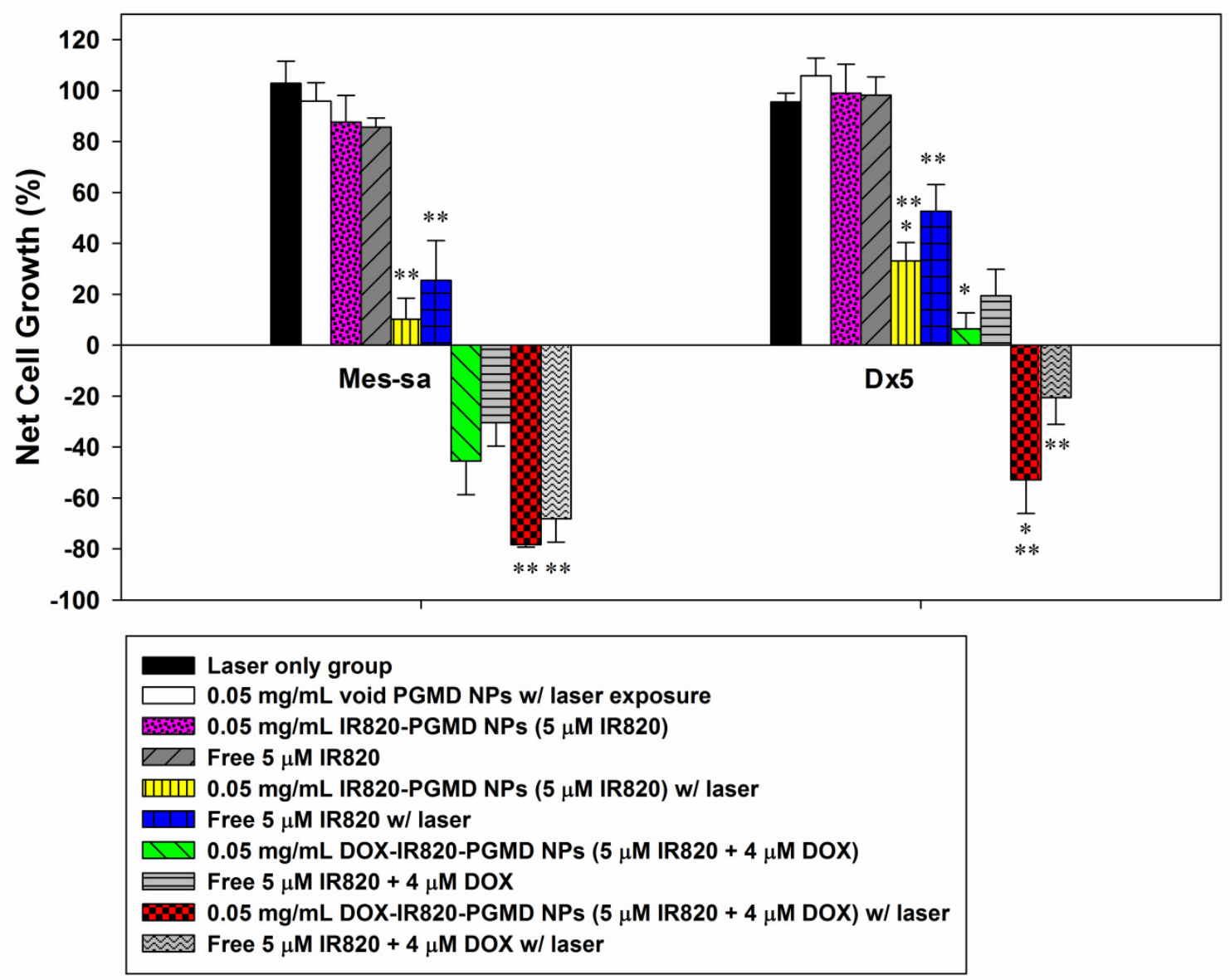

Figure 14. 24-hour cytotoxicity profile of NPs, free IR820 or free IR820 +free DOX w/ or w/o 3 minutes laser exposure in MES-SA and Dx5 cells; $n=3$, 4 wells/treatment. * $P<0.05$ (by ANOVA) indicates significant differences in cytotoxicity between free agent treatment and NPS groups in Dx5 due to the bypassing of P-gp; and $* * P<0.05$ (by 
ANOVA) indicates significant differences in cytotoxicity between laser-treated and nolaser-treated groups due to HT enhancement of cancer cell killing.

\subsection{Result accomplished for Specific Aim 2}

4.2.1. Cellular response after laser/Dye/NP HT and incubator HT

\subsubsection{ROS production after HT treatment}

ROS production after two different types of HT is shown in Figure 15. Incubator HT at $42^{\circ} \mathrm{C}$ for $1 \mathrm{~h}$ induced production of ROS in both MES-SA and Dx 5 cells, whereas ROS production after 3 minutes of $5 \mu \mathrm{M}$ laser/Dye/NP HT was not different from the control cells that were incubated in a $37^{\circ} \mathrm{C}$ incubator.

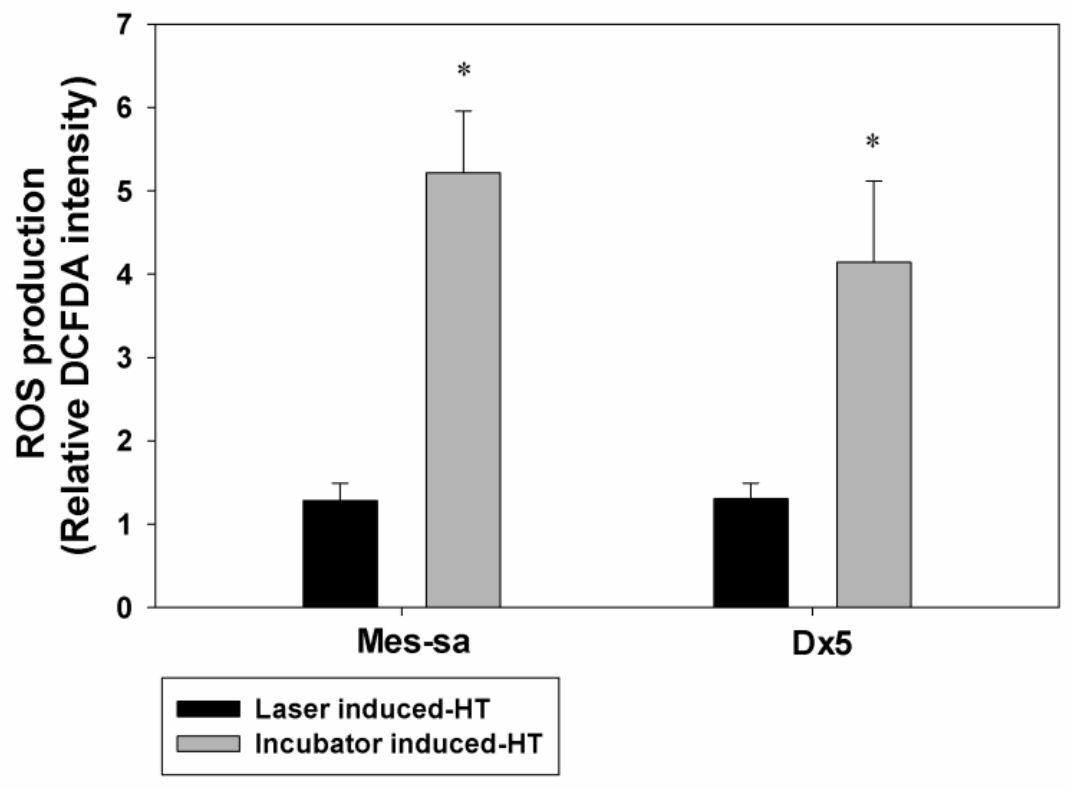

Figure 15. Laser/Dye/NP and incubator HT-induced ROS production were measured in MES-SA and Dx5 cells. Fluorescent dye CM-H2DCFDA was used to measure the 
fluorescence intensity and they were normalized to values obtained from the control group $\left(37^{\circ} \mathrm{C}\right) .{ }^{*} P<0.05$ indicates significant ROS production was observed in incubator induced-HT as compared to control. Laser/Dye/NP induced-HT did not result in enhanced ROS production as compared to control (mean $\pm S D, n=3$ ).

\subsubsection{HIF-1 expression}

As expected, incubator HT induced significantly elevated HIF-1 expression as compared to control $(\mathrm{p}<0.05)$, while laser/Dye/NP HT did not result in significant changes in HIF-1 expression. These results suggest that rapid laser/Dye/NP HT, with a much lower thermal dose, did not up-regulate HIF-1 expression.

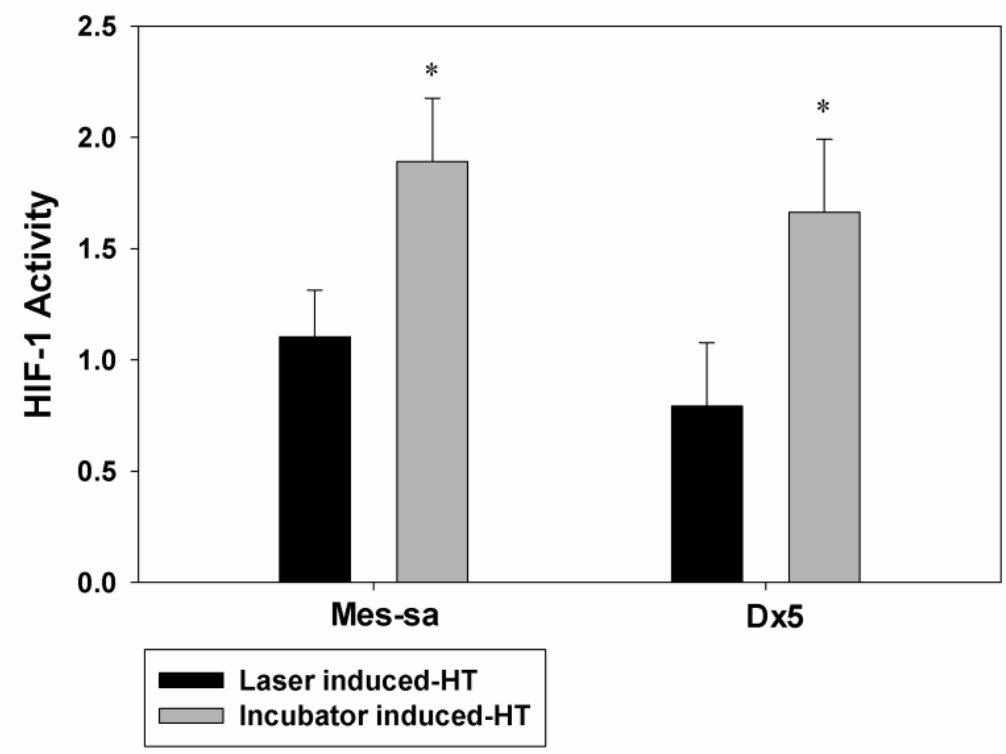

Figure 16. Laser/Dye/NP and incubator HT-induced HIF-1 expression were measured in MES-SA and Dx5 cells. HIF-1 activity was assayed using HIF-1 ELISA. All the values measured were normalized to the mean value of the treatment at $37^{\circ} \mathrm{C} .{ }^{*} \mathrm{P}<0.05$ 
indicates significant HIF-1 expression was observed in incubator induced-HT as compared to control. Laser/Dye/NP induced-HT did not result in promoted HIF-1 expression as compared to control (mean $\pm S D, n=3$ ).

\subsubsection{VEGF expression}

VEGF expression is shown in Figure 17. It is not surprising to observe that VEGF secretion was enhanced after incubator HT, since HIF-1 expression was elevated after incubator HT and VEGF is one of the downstream target genes of HIF-1. Accordingly, we did not observed significant changes in VEGF expression after laser/Dye/NP HT, given that laser/Dye/NP HT did not have any effect on HIF-1 expression.

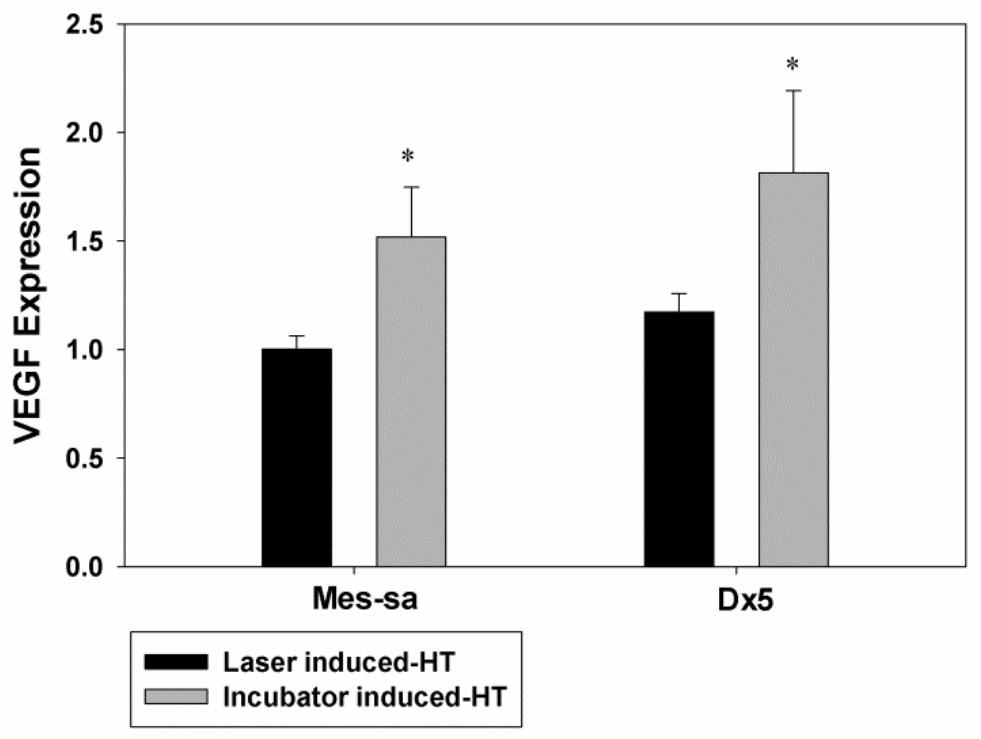

Figure 17. Laser/Dye/NP and incubator HT-induced VEGF expression were measured in MES-SA and Dx5 cells. VEGF secretion was measured using VEGF ELISA. The obtained VEGF expression amount was normalized to SRB value as an indicator of cellular 
protein amount. All the values measured were then normalized to the controls. $* P<0.05$ indicates significant VEGF expression was observed in incubator HT as compared to control. Laser/Dye/NP HT did not result in enhanced VEGF expression (mean $\pm S D, n=$ 3).

\subsubsection{Cytotoxicity study}

The laser/Dye/NP HT system described above was used for the cytotoxicity study. Significant cancer cell killing was achieved after exposure of IR820-PGMD NPs to this laser system. As shown in Figure 18, both incubator HT and laser/Dye/NP induced HT significantly killed cancer cells due to the HT effect $(p<0.05)$. Laser/Dye/NP HT significantly kills cancer cells better compared to incubator HT $(\mathrm{p}<0.05)$ probably due to the thermotolerance and cell protective mechanisms not being initiated in the latter group (106). 


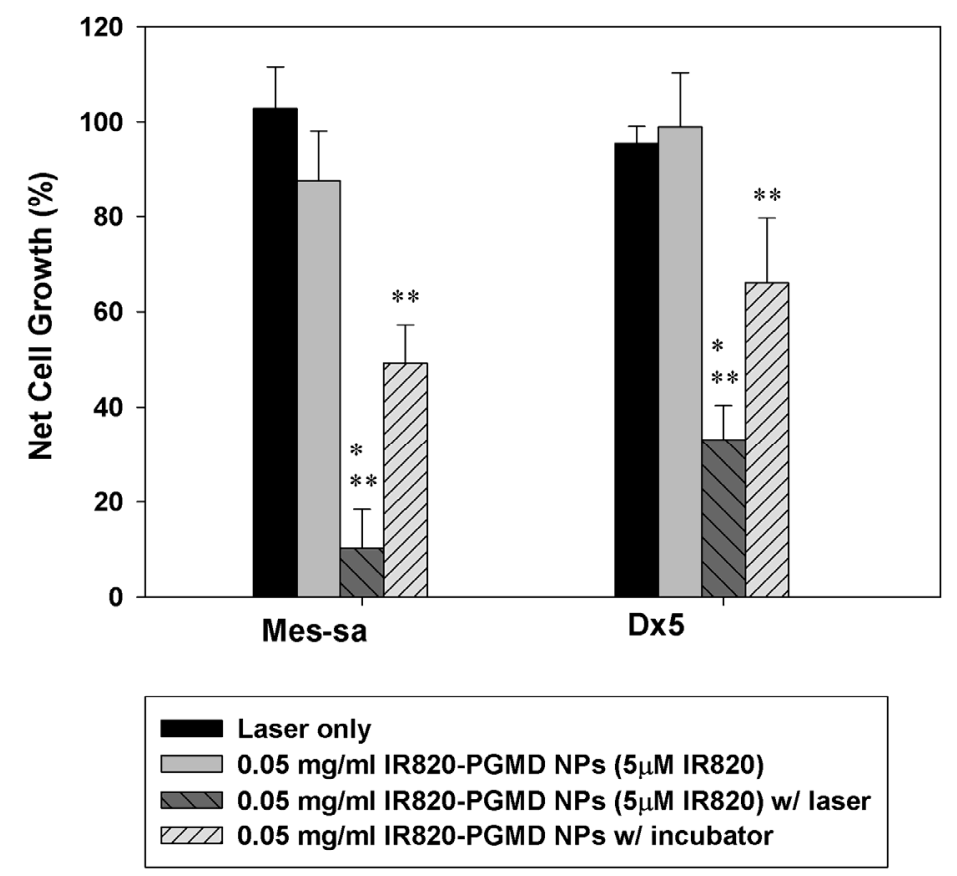

Figure 18. 24 hours cytotoxicity profile of IR820-PGMD NPs with laser and incubator exposure in MES-SA and Dx5 cells; $n=3,4$ wells/treatment. ${ }^{*} P<0.05$ (by ANOVA) between laser/Dye/NP HT and incubator/NP HT, indicating laser/Dye/NP HT results in significantly improved cytotoxicity compared to incubator HT. ${ }^{* *} P<0.05$ (by ANOVA) between HT groups and without HT group in both cell lines, indicating significantly higher cancer cell killing was achieved due to HT. 


\subsection{Results accomplished for Specific Aim 3}

4.3.1. Biodistribution and pharmacokinetic studies of IR820-PGMD NPs and DOXIR820-PGMD NPs

\subsubsection{In vivo optical imaging}

In vivo imaging was performed for all the time points mentioned in the Methodology section. The images taken at $15 \mathrm{~min}$ and $24 \mathrm{~h}$ are shown in Figures 19 . These images show that the biodistribution of IR820-PGMD NPs and DOX-IR820-PGMD NPs are initially very similar to free IR820, as both appear to be processed rapidly though hepatobiliary excretion accumulating in the liver within the first 15 minutes. After 24 hours, it seems that both free dye and NPs mainly locate in the liver and the organ studies showed that considerable IR820 content were also found in the kidney and the lungs, indicating uptake by RES. Image ratio $\mathrm{R}$ was calculate and given in Table 6, showing that NPs resulted in significantly higher IR820 fluorescence intensity than free IR820 24h after injection $(\mathrm{p}<0.05)$. Note that the image ratio $\mathrm{R}$ is only a measurement of normalized image intensity per pixel and cannot be used to quantitatively determine in vivo IR820 concentration. 

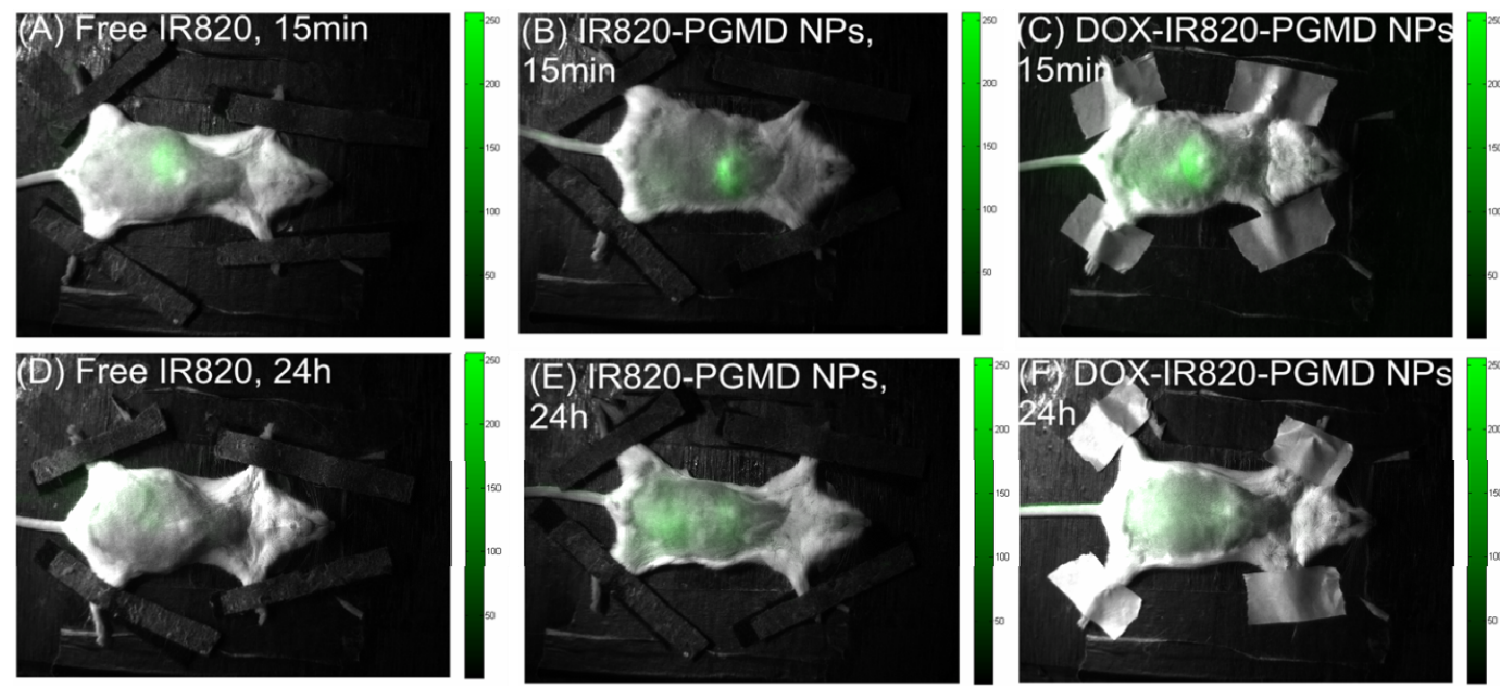

Figure 19. In vivo imaging of free IR820, IR820-PGMD NPs, and DOX-IR820-PGMD NPs. (A)-(C) 15 min in vivo imaging. (D)-(F) 24 h in vivo imaging.

\begin{tabular}{|l|l|l|}
\hline Treatments & $\mathrm{R}_{15 \min }$ & $\mathrm{R}_{24 \mathrm{~h}}$ \\
\hline Free IR820 & $1.97 \pm 0.47$ & $1.42 \pm 0.19$ \\
\hline IR820-PGMD NPs & $2.20 \pm 0.50$ & $* 2.37 \pm 0.62$ \\
\hline DOX-IR820-PGMD NPs & $1.88 \pm 0.54$ & $* 2.12 \pm 0.57$ \\
\hline
\end{tabular}

Table 6. Image ratio $R$ was calculated from fluorescence images of free IR820 and IR820-PGMD NPs treatments $(n=3) . * P<0.05$ indicates significant higher fluorescence intensity was observed in NPs as compared to free IR820 24 hours after injection.

\subsubsection{Pharmacokinetics studies}

The pharmacokinetics study of IR820 concentration in plasma at different time points after injection is shown in Table 7. The quantitative dye content analysis showed that 
IR820 was present in plasma in significantly higher amounts when carried in NPs compared to free IR820 24 hours after injection $(\mathrm{p}<0.05)$.

\begin{tabular}{|l|l|l|l|}
\hline & IR820 & IR820-PGMD NPs & DOX-IR820-PGMD NPs \\
\hline 15 minutes & $1.94 \pm 1.17$ & $3.63 \pm 0.20$ & $2.72 \pm 0.17$ \\
\hline 30 minutes & $0.97 \pm 0.15$ & $0.73 \pm 0.25$ & $1.00 \pm 0.21$ \\
\hline 60 minutes & $0.34 \pm 0.03$ & $0.29 \pm 0.02$ & $0.50 \pm 0.13$ \\
\hline 24 hours & $0.10 \pm 0.01$ & $* 0.18 \pm 0.05$ & $* 0.26 \pm 0.06$ \\
\hline
\end{tabular}

Table 7. Plasma concentrations $(\mu \mathrm{g} / \mathrm{mL})$ of IR820, IR820-PGMD NPs and DOX-IR820$P G M D N P$ s at different time points after injection. Values represent average $\pm S D(n=3)$. * $P<0.05$, indicates significant difference between both nanoformulations and free IR820 $24 h$ after injection.

Based on these results, it seems that NPs have advantages over free dye after initial distribution, which confirms our hypothesis that PGMD NPs can enhance the dye plasma residence time. The plasma concentration of DOX-IR820-PGMD NPs is also significantly higher than free IR820-PGMD NPs after 60min and 24h injection probably due to their differences in charge.

We also measured the DOX plasma concentration at different time points, the results are shown in Table 8. 


\begin{tabular}{|l|l|}
\hline Time/Treatments & DOX-IR820-PGMD NPs \\
\hline 15 minutes & $1.69 \pm 0.48$ \\
\hline 30 minutes & $1.37 \pm 0.16$ \\
\hline 60 minutes & $1.06 \pm 0.13$ \\
\hline 24 hours & $0.30 \pm 0.09$ \\
\hline
\end{tabular}

Table 8. Plasma concentrations $(\mu \mathrm{g} / \mathrm{mL})$ of DOX in DOX-IR820-PGMD NPs at different time points after injection. Values represent average $\pm S D(n=3)$.

\subsubsection{IR820 Pharmacokinetic modeling}

As discussed in the Methodology section 3.3.3.3., we assumed the initial plasma IR820 concentration is $4 \mu \mathrm{g} / \mathrm{mL}$. The IR820 plasma concentration of IR820, IR820-PGMD NPs and DOX-IR820-PGMD NPs after injection is shown in Figure 20 on a semi-log scale.

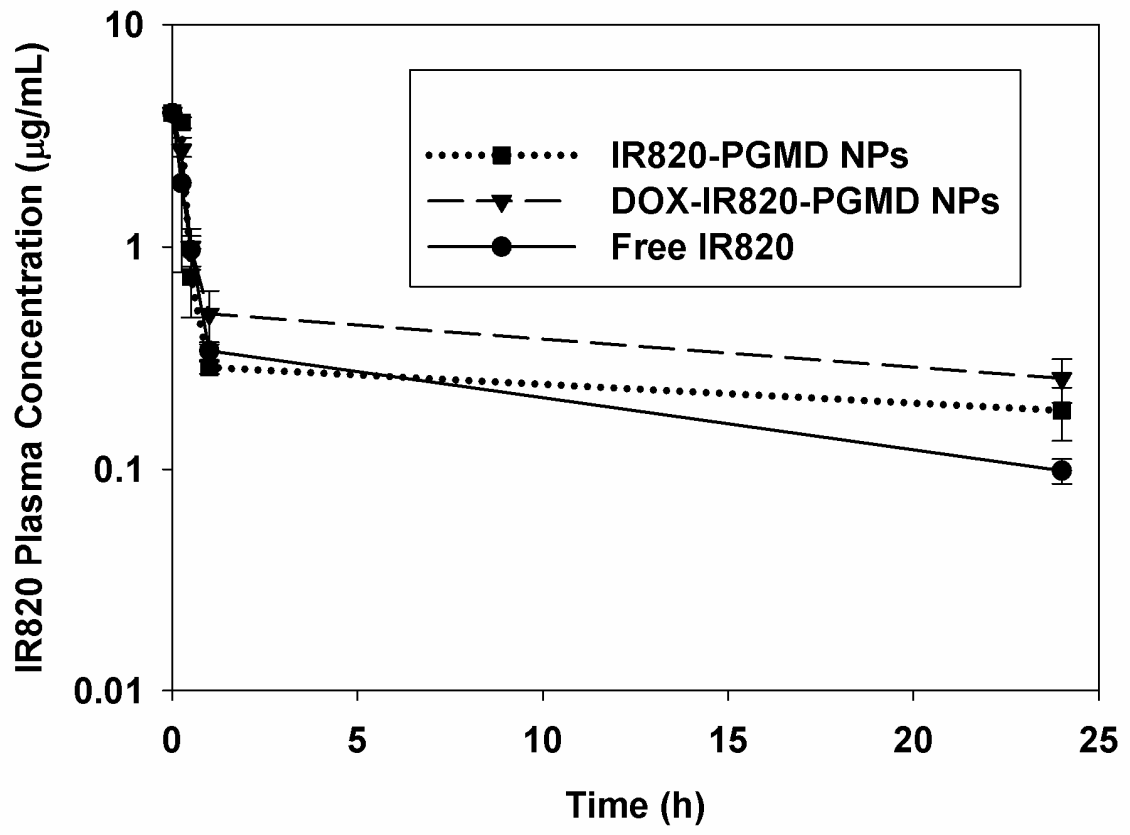


Figure 20. IR820 plasma concentration at different time points after i.v. injection of free IR820 or IR820 from NPs. The data are expressed in a semi-log scale.

We used both monoexponential and biexponential models to calculate the IR820 plasma half-life, AUC, mean plasma residence time, etc. The results are shown in Table 9 and Table 10.

a. One-compartment modeling

Table 9 shows the physiology parameters of IR820, IR820-PGMD NPs and DOX-IR820PGMD NPs calculated based on a one-compartment model.

\begin{tabular}{|l|c|c|c|}
\hline & IR820 & IR820-PGMD NPs & $\begin{array}{c}\text { DOX-IR820-PGMD } \\
\text { NPs }\end{array}$ \\
\hline Model equation & $\mathrm{C}(\mathrm{t})=3.984 \times \mathrm{e}^{-2.805 \mathrm{t}}$ & $\mathrm{C}(\mathrm{t})=4.344 \times \mathrm{e}^{-2.132 \mathrm{t}}$ & $\mathrm{C}(\mathrm{t})=4.099 \times \mathrm{e}^{-2.216 \mathrm{t}}$ \\
\hline $\mathrm{R}^{2}$ & 0.9971 & 0.8627 & 0.9674 \\
\hline
\end{tabular}

Table 9. Goodness of fit by using one-compartment model.

From the table above, we can see that the monoexponential model gives a good fit for our data. The coefficient of determination, denoted $\mathrm{R}^{2}$, is $0.99,0.86$, and 0.97 for IR820, I820-PGMD NPs, and DOX-IR820-PGMD NPs, respectively. The $\mathrm{R}^{2}$ is not optimal for IR820-PGMD NPs probably because of the small sample size or experimental error $(n=3$ for each data point). As more data points are acquired, the better experimental data fit is estimated. 


\section{b. Two-compartment modeling}

Table 10 shows the resulting parameters from a two-compartment modeling approach to obtain IR820, IR820-PGMD NPs, and DOX-IR820-PGMD NPs data.

\begin{tabular}{|l|c|c|c|}
\hline & IR820 & IR820-PGMD NPs & $\begin{array}{c}\text { DOX-IR820- } \\
\text { PGMD NPs }\end{array}$ \\
\hline Model equation & $\begin{array}{c}\mathrm{C}(\mathrm{t})=3.831 \times \mathrm{e}^{-3.108 \mathrm{t}} \\
+0.1702 \times \mathrm{e}^{-0.02275 \mathrm{t}}\end{array}$ & $\begin{array}{c}\mathrm{C}(\mathrm{t})=2.217 \times \mathrm{e}^{-2.132 \mathrm{t}} \\
+2.127 \times \mathrm{e}^{-2.132 \mathrm{t}}\end{array}$ & $\begin{array}{c}\mathrm{C}(\mathrm{t})=2.066 \times \mathrm{e}^{-2.216} \\
+2.033 \times \mathrm{e}^{-2.216 \mathrm{t}}\end{array}$ \\
\hline $\mathrm{R}^{2}$ & 0.9999 & 0.8507 & 0.9524 \\
\hline
\end{tabular}

Table 10. Goodness of fit by using two-compartment model.

We used both monoexponential and biexponential models to estimate free dye and NPs' physiology parameters. It is shown that $\mathrm{R}^{2}$ was not improved significantly by changing one-compartment model to two-compartment model for estimation of free IR820 pharmacokinetics, since $\mathrm{R}^{2}$ is already 0.99 with monoexponential model. On the other hand, $\mathrm{R}^{2}$ for both NP formulations decreased to 0.85 and 0.95 as compared to the onecompartment model, respectively. Therefore, it seems the biexponential model did not result in improvements for assessment of model accuracy, although the elimination of IR820 seems to follow a biexponential pattern. This is probably because the goodness of fit is not very good for IR820 loaded NPs plasma data due to the reason that NPs may have a different distribution pattern, if we take into account of the IR820 release kinetics from the NPs. Therefore, we decided to start from the simplest model and use the onecompartment model to calculate all the pharmacokinetics parameters.

Using monoexponential model, we can calculate all the physiology parameters as shown in Table 11. 


\begin{tabular}{|l|l|l|l|}
\hline & Free IR820 & $\begin{array}{l}\text { IR820- } \\
\text { PGMD NPs }\end{array}$ & $\begin{array}{l}\text { DOX-IR820- } \\
\text { PGMD NPs }\end{array}$ \\
\hline Elimination half-life (hours) & $0.25 \pm 0.005$ & $0.33 \pm 0.022^{*}$ & $0.31 \pm 0.006^{*}$ \\
\hline AUC $\left[\mu \mathrm{g}^{*} \mathrm{~h} / \mathrm{mL}\right]$ & $1.42 \pm 0.028$ & $2.04 \pm 0.137^{*}$ & $1.85 \pm 0.038^{*}$ \\
\hline Mean plasma residence time, $\mathrm{t}_{\mathrm{p}}(\mathrm{h})$ & $0.36 \pm 0.007$ & $0.51 \pm 0.034^{*}$ & $0.46 \pm 0.009^{*}$ \\
\hline Total body clearance rate $(\mathrm{mL} / \mathrm{h})$ & $4.22 \pm 0.084$ & $2.94 \pm 0.198^{*}$ & $3.24 \pm 0.066^{*}$ \\
\hline
\end{tabular}

Table 11. Pharmacokinetic parameters calculated based on one-compartment analysis of dye data in mice. Data represent mean $\pm S . D .{ }^{*} P<0.05$, indicates significance difference between NPs and free IR820.

As expected, NPs resulted in longer elimination half-lives, larger AUC, longer plasma residence times, and slower body clearance rates than free IR820. The monoexponential calculated plasma half-lives for free IR820, IR820-PGMD NPs, and DOX-IR820-PGMD NPs were approximately 14.5 minutes, 19.5 minutes, and 18.7 minutes, respectively. The bioavailability of IR820 is increased by using NPs, as the elimination half-life of IR820PGMD NPs and DOX-IR820-PGMD NPs is 1.4 times and 1.3 times the free IR820 elimination half-life, respectively. All the calculated physiology parameters indicated prolonged blood circulation with nanoformulation and the cumulative exposure to body organs is also enhanced, similar results were also reported in other studies $(127,128)$.

\subsubsection{DOX pharmacokinetics modeling}

Based on the loading efficiency of DOX in DOX-IR820-PGMD NPs, and an IR820 dose of $0.24 \mathrm{mg} / \mathrm{kg}$, the initial concentration of injected DOX in plasma was approximately 2 $\mu \mathrm{g} / \mathrm{mL}$. Therefore, the DOX plasma concentration of DOX-IR820-PGMD NPs at different time points after injection was plotted in Figure 21. 


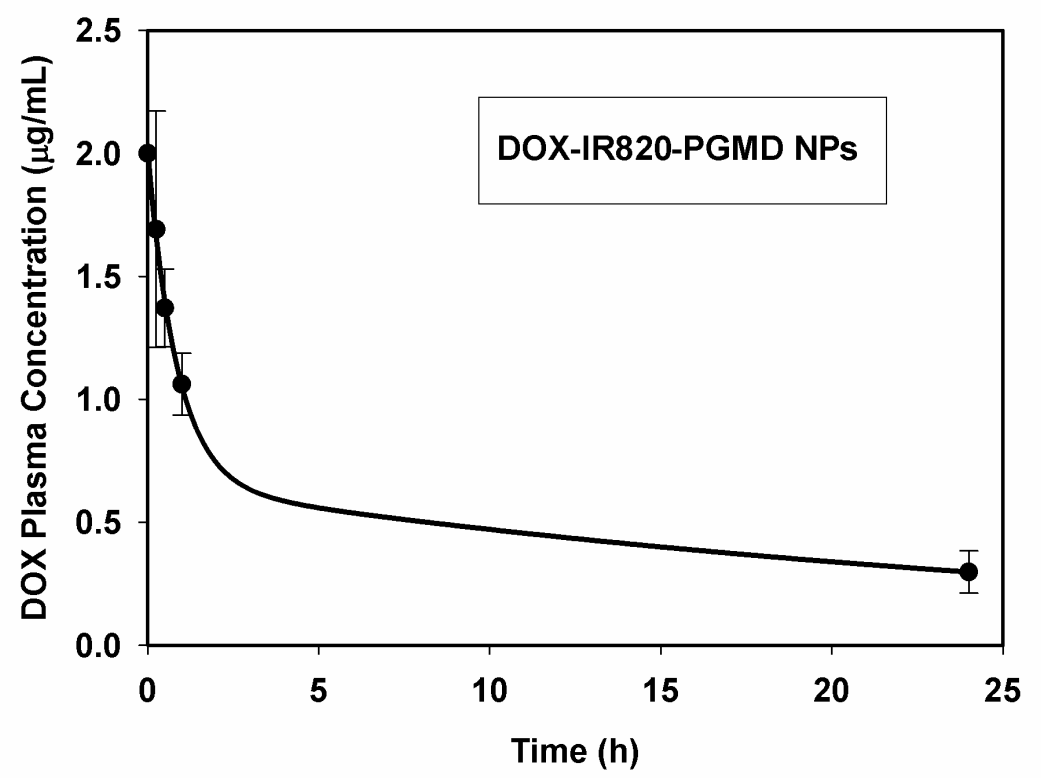

Figure 21. DOX plasma concentration at different time points after i.v. injection in DOXIR820-PGMD NP treatment.

We also used both one-compartment model and two-compartment model to assess the goodness of model fit to the experimental data as shown in Table 12.

\begin{tabular}{|l|l|l|}
\hline & One-compartment model & Two-compartment model \\
\hline Model equation & $\mathrm{C}(\mathrm{t})=1.985 \times \mathrm{e}^{-0.66 \mathrm{t}}$ & $\mathrm{C}(\mathrm{t})=1.356 \times \mathrm{e}^{-1.167 \mathrm{t}}+0.6535 \times \mathrm{e}^{-0.0327 \mathrm{t}}$ \\
\hline $\mathrm{R}^{2}$ & 0.9453 & 0.9989 \\
\hline
\end{tabular}

Table 12. Goodness of fit by using one or two-compartment model.

As shown in Table 12, it seems that the biexponential model may provide a better understanding of our data as $\mathrm{R}^{2}$ was improved from 0.94 to 0.99 when a biexponential model was used instead of a monoexponential model. Moreover, the introduction of 
distribution phase also gives us insight into the DOX-IR820-PGMD NPs distribution and elimination behavior. Hence, we decided to calculate all the physiology parameters following the use of a two-compartment model. All the values are shown in Table 13.

\begin{tabular}{|l|c|}
\hline Distribution half-life (hours) & $0.59 \pm 0.0008$ \\
\hline Elimination half-life (hours) & $21.2 \pm 0.028$ \\
\hline AUC $\left[\mu \mathrm{g}^{*} \mathrm{~h} / \mathrm{mL}\right]$ & $12.03 \pm 0.016$ \\
\hline Mean plasma residence time, $\mathrm{t}_{\mathrm{p}}(\mathrm{h})$ & $3.01 \pm 0.004$ \\
\hline Total body clearance rate $(\mathrm{mL} / \mathrm{h})$ & $0.5 \pm 0.0007$ \\
\hline
\end{tabular}

Table 13. Pharmacokinetic parameters from two-compartment analysis of dye data in mice. Data represent mean \pm S.D.

From Table 13, the calculated plasma half-lives were approximately 36 min (distribution half-life) and 22 hours (elimination half-life). It seems DOX plasma half-life is also enhanced when they are encapsulated in NPs. This is a significant improvement over literature reports for free DOX which described a distribution half-life of $\sim 2$ minutes and elimination half-life of $\sim 10.3$ hours in mice (129).

\subsubsection{Organ dye content}

Table 14 shows the average dye content in $\mu \mathrm{g}$ dye/g tissue for liver, lungs, intestines and kidneys 24 hours after an i.v. injection of IR820, IR820-PGMD NPs, and DOX-IR820PGMD NPs. Our organ studies showed that IR820, both when in free form and encapsulated into NPs, are processed primarily by hepatobiliary excretion and starts to accumulate in the liver within 5-10 minutes after injection. However, organs such as the 
kidney and the lungs also have considerable contents of IR820 after 24 hours, indicating uptake by the RES. DOX-IR820-PGMD NPs demonstrate significantly lower dye content in the kidneys compared to the free dye ( $\mathrm{p}=0.04)$, and 2.6 times smaller dye content in the liver, although the latter did not reach a statistically significant difference from free IR820 ( $\mathrm{p}=0.08)$. As for the IR820-PGMD NPs, dye content was lower in kidneys and lungs, but the difference also did not reach statistical significance. The lack of statistical significance is probably due to the small number of subjects used in the study as well as individual variability. DOX organ content after 24 hours injection was not detectable in any tissue samples; that is, the DOX fluorescence intensity was not greater than the background autofluorescence, probably due to the sub-therapeutic DOX dose used in this study. Based on the results, we can say that DOX-IR820-PGMD NPs demonstrate decreased renal clearance compared to free dye.

\begin{tabular}{|l|l|l|l|l|}
\hline $\begin{array}{l}\text { 24h organ and plasma IR820 dye } \\
\text { content }(\mathrm{n}=3)\end{array}$ & $\begin{array}{l}\text { Liver } \\
(\mu \mathrm{g} / \mathrm{g})\end{array}$ & $\begin{array}{l}\text { Lungs } \\
(\mu \mathrm{g} / \mathrm{g})\end{array}$ & $\begin{array}{l}\text { Intestines } \\
(\mu \mathrm{g} / \mathrm{g})\end{array}$ & $\begin{array}{l}\text { Kidneys } \\
(\mu \mathrm{g} / \mathrm{g})\end{array}$ \\
\hline IR820 & $0.21 \pm 0.11$ & $0.28 \pm 0.03$ & $0.05 \pm 0.006$ & $0.41 \pm 0.11$ \\
\hline IR820-PGMD NPs & $0.21 \pm 0.09$ & $0.23 \pm 0.07$ & $0.07 \pm 0.02$ & $0.25 \pm 0.09$ \\
\hline DOX-IR820-PGMD NPs & $0.08 \pm 0.01$ & $0.26 \pm 0.08$ & $0.05 \pm 0.02$ & $* 0.21 \pm 0.04$ \\
\hline
\end{tabular}

Table 14. Quantitative organ content 24 hours after i.v. injection of IR820, IR820-PGMD NPs, and DOX-IR820-PGMD NPs. Values represent average $\pm S D .{ }^{*} P<0.05$ indicates significant difference between DOX-IR820-PGMD NPS and free IR820 values for kidneys. 


\section{CHAPTER 5: DISCUSSION AND CONCLUSIONS}

\subsection{Characterization of PGMD polymer}

The MW of PGMD polymer is around $3000 \mathrm{Da}$, which is consistent with the literature stating that polycondensation of monomers would preferentially yield low MW polymers (130). When high MW polymers are desired, ring opening polymerization is preferred (131). Jesus et al. synthesized a polyester dendritic scaffold based on the monomer unit (2,2-bis(hydroxymethyl)propanoic acid), and measured a polymer MW around $4000 \mathrm{Da}$ (132). The PGMD NPs we obtained are in the 100-150 nm range, which can potentially avoid premature clearance by the reticuloendothelial system (133). The loading of IR820 is sufficient to induce HT without causing cytotoxicity by NPs themselves. Loading of IR820 and DOX into the PGMD NPs increased the PDI of the NPs as compared to void NPs. Cheng et al. reported that increased PDI was observed with increasing loading amount of docetaxel into PLGA-PEG (polyethylene glycol) NPs (134). The observed increase in DOX-IR820-PGMD NPs zeta potential after addition of DOX and IR820 as compared to void PGMD NPs may be caused by a zeta potential change towards neutral due to incorporated DOX amino groups.

\subsection{In vitro release of DOX and IR820}

In our previous work, we developed a multifunctional system by loading ICG (as imaging/HT agent) and DOX (chemotherapy agent) into PLGA NPs. Our studies showed that ICG-DOX-PLGA NPs are able to bypass the P-gp pump in MES-SA/Dx5 cells and are able to induce a synergistic effect by enhancing the cancer cell killing compared to 
chemotherapy alone or HT alone (106). However, DOX release from ICG-DOX-PLGA loaded NPs is very slow with approximately $50 \%$ still retained in the NPs after 30-day incubation in $\mathrm{pH}=7.4$ phosphate buffered saline. The DOX release profile was not improved even after exposing the NPs to NIR laser, which elevated the temperature to $\sim 43^{\circ} \mathrm{C}$ due to the presence of the ICG. It seems that PLGA NPs are not sensitive to external heat $\left(\sim 43^{\circ} \mathrm{C}\right)$ probably due to the high $\operatorname{Tg}\left(45^{\circ} \mathrm{C}-50^{\circ} \mathrm{C}\right)$ and high MW $(40,000$ $75,000 \mathrm{Da})$ of the PLGA used, which could have a large effect on the release rate (135). Furthermore, the unmodified PLGA is hydrophobic, which in many cases limits its applications to encapsulation of hydrophobic drugs.

The release of DOX from PGMD NPs was increased after exposure to laser. This is perhaps because of the phase change of PGMD polymer $\left(T g=42.2^{\circ} \mathrm{C}\right)$ at high temperatures, which increases the release of DOX from the polymer matrix. There are several synthetic polymers sensitive to temperature change, such as acrylamide-based hydrogels, especially poly[N-isopropylacrylamide] (PNIPA) hydrogel, and elastin-like polypeptides (136-138). Zhang et al. reported that a synthetic PNIPA hydrogel releases $20-30 \%$ more of its 5 -fluorouracil load at $37^{\circ} \mathrm{C}$ compared to $10^{\circ} \mathrm{C}$ (139). In addition, acidic environment can also induce higher DOX and IR820 release, probably due to the accelerated hydrolysis of PGMD polymer (140).

It is well documented that tumor interstitium has lower $\mathrm{pH}$ than blood and healthy tissue $(141,142)$. Therefore, a rapid release of DOX from PGMD NPs in an acidic environment could be beneficial in cancer therapy. Moreover, the heating of IR 820 by an external NIR laser can further induce release of DOX at tumor site. Generally, DOX release from the 
NPs was faster in the burst release phase as compared to our previous study of DOX release from PLGA NPs (143), which is probably due to the fact that PGMD has a much lower molecular weight compared to the PLGA used in that study ( $3 \mathrm{kDa}$ v.s. $75 \mathrm{kDa})$. Zolnik reported that polymer MW is a key factor in determining release rate, and slow release was observed in high MW polymers (70 kDa) (135). On the other hand, PGMD is more hydrophilic than PLGA due to the addition of malic acid in the polycondensation process. Thus, when using these two polymers to synthesize NPs, the PLGA NPs are estimated to have a stronger hydrophobic-hydrophobic interaction with DOX than PGMD NPs do, which could be another reason that a higher amount of DOX was released from PGMD NPs compared to PLGA NPs for the same time period.

After exposure of the NPs to both acidic environment and laser irradiation, we observed a further enhanced release of DOX from PGMD NPs. This is probably because of the combination of physical and chemical effects as described above. The temperature of NPs after laser exposure can increase the PGMD polymer $T g$, which may cause the NPs' physical properties to change resulting in the transition of amorphous materials from noncrystalline solid, relatively hard state to a molten state. The physicial property changes could possibly soften the polymer and enlarge the NPs pore size, which may result in a nonreversible increase in polymer hydrolysis since it becomes easier for water molecules to penetrate the polymer and swell the particles. The enhanced hydrolysis could accelerate the degradation of the polymer and induce higher diffusion of DOX from the polymer matrix. 


\subsection{Subcellular localization of DOX and IR820 in DOX-IR820-PGMD-NPs and Uptake of NPs}

In the subcellular localization study, DOX fluorescence was detected in both the cytosol and the nucleus. We have demonstrated that the fate of PGMD NPs is mainly localizing in lysosomes. Some of the NPs are able to escape endolysosomal degradation and release their payload in the cytosol. The escape process primarily takes place through selective reversal of the NPs' surface charge (from anionic to cationic) in the acidic endolysosomal compartment, causing the NPs to interact with the endo-lysosomal compartment membrane and to escape into the cytosol (144). The subcellular localization of IR820 in both IR820-PGMD NPs and DOX-IR820-PGMD NPs treatments is very similar since IR820, once released from NPs, possibly binds to cytoplasmic protein such as ligandin (125).

The cellular uptake of NPs could be affected by their charge and shape. Zhang's group reported that spherical NPs had a higher cell entry rate into Chinese hamster ovary cells than cylindrical NPs (145), whereas Chithrani et al. reported a lower uptake of rodshaped gold NPs into HeLa cells compared to spherical shaped NPs (146). On the other hand, Liang et al. and Chung et al. reported that slight positively charged NPs gave the greatest uptake efficiency as compared to neutral and negatively charged NPs $(147,148)$. It seems spherical shape and slightly positive NPs are more efficiently taken up by cells compared to other shapes and negatively charged NPs. These studies show that the selection of the NPs are very important and can influence the NPs uptake by cells. Hence, 
the optimization of such parameters should be considered in the design of NPs in order to improve the NPs delivery for various biomedical applications.

\subsection{Cytotoxicity}

The NPs result in significantly higher cell killing than does free IR820 or free IR820 plus DOX in Dx5, but not in MES-SA cells. This is because MES-SA is a sensitive cell line which does not overexpress P-gp, so the NPs do not provide an advantage in increasing cellular uptake over the drug's free form. All these results are in accord with the cellular uptake study, in which we observed comparable DOX cellular uptake of DOX-IR820 and DOX-IR820-PGMD NPs in MES-SA cells, whereas when the DOX was encapsulated in the NPs, the result was a much higher uptake of DOX in Dx5 cells compared to the uptake of DOX without the NPs. Our previous study of DOX-PLGA NPs had shown that NPs can overcome the P-gp pump efflux effect and increase the uptake and cytotoxicity in MDR cell lines, because the NPs formulation can protect the drug from being recognized by the P-gp pump (35). Our current study result also showed that the drug loaded NPs kill more Dx5 cells after exposure to laser as compared to free IR820 or free IR820 plus DOX $(\mathrm{p}<0.05)$. Improved cancer cell killing can be achieved with a combination of HT and chemotherapy. Our previous study demonstrated that mild cell apoptosis can be induced by mild HT (106). Furthermore, the therapeutic effect of DOX can be potentially augmented because mild HT can enhance cell membrane permeability and fluidity, and in turn result in greater accumulation of the drug inside cancer cells, especially for MDR cancer cells. 
In this study, we used two different heating modalities: 3 minutes laser/Dye/NP HT and 1 hour incubator HT, and the thermal doses for each were calculated to be approximately 3 minutes and 26 minutes, respectively, based on the $\mathrm{CEM}_{42}$ model. The model was modified from the commonly used $\mathrm{CEM}_{43}$ model. IR820 was still able to cause a temperature increase after laser exposure, even after being encapsulated into NPs, which is comparable to the temperature increase achieved with free IR820. Thus, it was shown in cytotoxicity studies that laser/Dye/NP induced HT caused significantly higher cell killing than incubator HT, although a much lower thermal dose was given to the cells (approximately 9 times less than with incubator HT). In the commonly used thermal dose $\mathrm{CEM}_{43}$ model, in which thermal dose is normalized to cumulative equivalent minutes at $43^{\circ} \mathrm{C}(110)$, temperature and duration of heating can be used to define thermal damage. Our previous paper and other groups' reports demonstrated that the rate of photothermal treatment might also be affecting HT outcome, since the cells are not able to initiate the protective mechanism by inducing heat shock protein expression which would help to reduce DNA damage $(55,149)$. Although the laser/Dye/NP HT produced approximately 9 times less thermal dose than incubator HT, it still resulted in significantly higher cytotoxicity than incubator HT, thus confirming the importance of heating rate. Note that the final temperature reached in both modes of HT was identical.

\subsection{Study of ROS, HIF-1, and VEGF}

Following the cytotoxicity study, we were interested in investigating the effect of rate and amount of thermal dose to cells by exploring ROS generation, HIF-1 and VEGF expression. HIF-1, as an important therapeutic target gene for cancer therapy, was studied 
after two different modes of HT. The purpose of this study was to find out what would be a better strategy to apply HT and achieve the ideal therapeutic outcome.

It has been reported by Madamanchi's group that ROS can up-regulate HSP70 protein levels through the activation of binding of signal transducers and activators of transcription (STATs) to the HSP70 promoters in vascular smooth muscle cells (VSMCs) (105). This group exposed VSMCs to $\mathrm{H}_{2} \mathrm{O}_{2}$ and found that the cytoplasmic janus tyrosine kinase 2 (JAK2)/STAT pathway can up-regulate HSP70 and minimize oxidative stress effects on the cells. The inhibition of HSP70 expression under laser/Dye/NP HT probably means no enhancement of ROS production within the cells. Our ROS detection experiments support this hypothesis, showing that no significant ROS was produced inside the cells after laser/Dye/NP HT as compared to controls. However, when incubator HT was used to mimic conditions more similar to whole body HT, we observed significant intracellular ROS production. This result is consistent with Moon et al. reporting that ROS was activated when a slow waterbath HT was applied to cells. HT can activate the ERK pathway and increase NADPH oxidase activity, which leads to the production of ROS (150). Based on our results, it seems that the application of rapid laser/Dye/NP HT to cells will not induce an increase of ROS. However, the specific mechanism of ROS abolishment within cells after laser/Dye/NP HT has to be further studied.

Since there is no activation of ROS production in laser/Dye/NP HT treatment, we did not observe enhanced HIF-1 expression either. However, HIF-1 up-regulation was observed in slow and longer term HT, probably because ROS production was activated in the 
heating process. Other groups have also suggested that the presence of ROS is able to upregulate HIF-1 expression $(99,151)$. HIF-1 is very important as a therapeutic target (152). Though traditional HT with slow and long-term heating appears beneficial as an adjuvant therapy for radiotherapy and chemotherapy since it can hinder DNA damage repair mechanisms and increase drug delivery $(153,154)$, this heating modality is also able to induce up-regulation of HIF-1, and the overexpression of HIF-1 could compromise the therapeutic effect by increasing drug resistance through up-regulation of p-glycoprotein, and by reducing cancer cells drug senescence $(155,156)$. Our results showed that VEGF secretion was also elevated along with the up-regulation of HIF-1, which could potentially result in enhanced tumor angiogenesis.

The combination of HT and other therapies could elevate the HIF-1 expression to an even higher extent than just a single therapy, which could alter tumor cell behavior and make cells more aggressive. Therefore, it is important to review the possible molecular effects of HT in considering its application as an adjuvant therapy, since other groups have reported that HIF-1 can also be up-regulated by radiotherapy and chemotherapy (157159). Based on our study, IR820-PGMD NPs could be used for HT applications without inducing the adverse effects of HIF-1. The HT therapeutic effect would be determined more by the temperature and heating rate and perhaps less on the total thermal dose. By using laser/Dye/NP HT, we did not observe enhancement of HIF-1 and VEGF expression, but an improved therapeutic outcome was still achieved compared to incubator HT. Despite these promising results for laser/Dye/NP HT, further studies 
should be performed to determine treatment parameters, such as how to efficiently deliver these NPs and the timing for HT treatment relative to the chemotherapy.

\subsection{In vivo studies}

The nanoparticles we synthesized had a size around $100 \mathrm{~nm}$ diameter, which is considered as an optimal size for particles and could possibility prolong the blood circulation compared to larger or smaller particle sizes according to literatures reports (127) (160). Therefore, it seems that our NPs should have an increased circulation time profile as compared to their drug's free form. In vivo imaging studies were done in healthy mice. The mice imaging showed that NPs formulation enhanced IR820 fluorescence intensity as compared to free IR820 after 24 hours $(\mathrm{P}<0.05$ for in vivo imaging studies). This is consistent with the literature reporting that nanoformulation or liposomes can result in improved plasma circulation time and protect the loading agent from degradation, which probably leads to the enhanced fluorescence intensity as compared to free form $(127,161)$.

Our previous in vitro fluorescence intensity studies showed that the NPs resulted in about $8 \%$ fluorescence intensity decrease as compared to free IR 820 at the same IR 820 concentration, which may be due to the increased scattering within NPs. We have observed similar phenomenon in our previous study of covalent IR820-PEG diamine nanoconjugates (162). However, the stability of IR820 was enhanced as compared to free

IR820 as can be seen in Table 11. Our pharmacokinetics study showed that plasma has significantly higher IR820 concentration in NPs form than free IR820 24h after injection. 
Based on available literature reports, nanoformulations also seem to result in improved pharmacokinetic profiles, in many cases as a result of their size and surface properties, ability to stabilize encapsulated drugs/dyes, and reduced liver metabolism and renal clearance $(127,161)$. In addition, our biodistribution study showed that kidney IR820 dye content was lower in NPs form than free IR820, and the difference between DOX-IR820PGMD NPs and free IR820 is significant, which means less IR820 was excreted through the renal system when encapsulated in the NPs. When a one-compartment model was used to study the physiology parameters of free IR820 and IR820 encapsulated NPs (both DOX-IR820-PGMD NPs and IR820-PGMD NPs), the IR820 encapsulated NPs resulted in much longer elimination half-lives, longer mean plasma residence time, larger overall exposure as indicated by AUC, and slower clearance rate compared to free IR820. The increased IR820 plasma half-life and prolonged circulation time in NPs formulation may present an advantage over the free form by stabilizing the dye and allowing longer image collection periods in imaging studies. Additionally, a widened therapeutic window may be available when providing HT as an adjuvant therapy, thanks to prolonged exposure of tissues to IR820. Since the EPR effect is proportional to the time and amount of drug/dye circulating in blood (122), it would be reasonable for us to expect that NPs formulation should result in higher accumulation and retention of IR820 in tumors. However, further studies should be performed in a tumor-bearing model to support our hypothesis.

DOX plasma half-life is enhanced when it is encapsulated in NPs. Literature reports for the plasma half-life of free DOX is $\sim 2$ minutes and elimination half-life of $\sim 10.3$ hours in mice (129). Other researchers have also observed prolonged DOX plasma half-lives in 
different animal models when a nanoformulation, such as liposomes or NPs, was used (119-121). For instance, Reddy et al. reported that DOX loaded poly(butyl cyanoacrylate) NPs prolonged DOX half-life $\sim 1.5$ fold as compared to free DOX in rats (128). One of the most important DOX-loaded liposome applications in clinical cancer therapy is DOXHCL encapsulated in pegylated liposomes for i.v. injection $\left(\mathrm{DOXIL}^{\circledR}\right)$. The pharmacokinetics studies of DOXIL ${ }^{\circledR}$ in tumor-bearing mice followed a biexponential curve, with the first distribution half-life of 1-3 hours, and a second elimination phase half-life of 30-90 hours $(121,163,164)$. Although DOX-IR820-PGMD NPs seem to have shorter plasma half-life as compared to DOXil, they could also be pegylated and thus longer plasma circulation time would be expected (165-167). The increased DOX plasma half-life could have an impact on the therapeutic efficacy, because higher overall exposure and prolonged exposure profiles can result in enhanced in vivo tumor uptake and improved therapeutic efficacy.

\subsection{Conclusions}

In this study, multifunctional PGMD NPs were successfully synthesized to be used to combine different therapeutic techniques such as chemotherapy and HT. The novelty of this study is the synthesis of a thermal and $\mathrm{pH}$ sensitive polymer which provides a controllable and predictable pharmacokinetic release profile using thermal or $\mathrm{pH}$ stimuli. This novel and adjustable delivery vehicle was loaded with the chemotherapy agent DOX and the imaging and HT agent IR820. The resulting NP formulations can be used to improve cellular uptake and cytotoxicity in the MDR cancer cell line Dx5. The combination of chemotherapy and HT also enhanced DOX cytotoxicity in both MES-SA 
and Dx5 compared to single therapy alone, indicating that less DOX can be used when "adjuvant" cancer therapy is introduced. In vitro and in vivo studies showed that the NPs yield higher IR820 fluorescence intensity than free IR820 after 24 hours, allowing longer imaging collection times and potentially widening the window for HT applications. Additionally, we proved in our study that using IR820-PGMD NPs, laser/Dye/NP HT will not activate ROS expression and therefore would not induce HIF-1 and VEGF expression. This lack of activation could yield a beneficial therapeutic outcome. In vivo studies showed that the IR820 in NP formulation has a longer plasma half-life than free IR820, providing longer imaging collection times for cancer diagnostics, and potentially widening the window for HT applications. An increase in DOX plasma half-life was also observed in NPs formulation, which could possibly result in an increased exposure of tumor cells to the chemotherapeutic drug; coupled with the passive targeting provided by the enhanced permeability and retention (EPR) effect may increase tumor uptake (168). This could potentially lead to improvements in therapeutic efficacy. Thus, IR820-PGMD NPs and DOX-IR820-PGMD NPs have promising applications as theranostic agents with multifunctional imaging, HT and chemotherapy capabilities. This study is an extension to the current knowledge of delivery of in vivo imaging probe, HT and chemotherapy in NPs form, and we believe it will have significant impact to the application of nanotechnology on cancer imaging and therapy.

\subsection{Limitations and future work}

Although the PGMD NPs seem to be promising in cancer diagnostic and therapy, more studies have to be performed in order to confirm our hypothesis. 
$1^{\text {st }}$. The FTIR spectrum only shows that presence of functional group $\mathrm{C}=\mathrm{O}$ bond, indicating polyester is forming in the PGMD polymer preparation, however it can not give any information regarding to the composition of malic acid and DDA in PGMD polymer. Polymer synthesis through thermal condensation method is a process called step-growth polymerization by connecting two molecules and resulting in loss of small molecules, which in our case is water. We have indirect evidence showing that malic acid is included in the PGMD polymer and the change of DDA to malic acid ratio from 7:3 to 6:4 decreases the PGMD polymer $\operatorname{Tg}$ to between $40-41^{\circ} \mathrm{C}$. However, Nuclear Magnetic Resonance (NMR) spectroscopy should be acquired in order to address the questions of PGMD polymer composition in the future work.

$2^{\text {nd }}$. For the cellular response studies after heating by two different HT modalities, the mechanism of inhibited ROS production after laser/Dye/NP HT is not clear and should be further studied to prove that the ROS production inhibition is due to the small thermal dose or due to the rapid thermal heating rate, or the effect of both. In terms of molecular biology, different inhibitors should be used to block corresponding ROS production pathways and to find out exactly which pathway is used by cells to suppress the ROS production.

In addition, the measurement of HIF-1 and VEGF expression after different HT were done in cells, which might provide valuable but limited information due to the large difference between an in vitro and in vivo model. Therefore, in vivo model with tumorbearing mice should be used to further study the in vivo HIF-1 and VEGF expression. 
$3^{\text {rd }}$. Although PGMD NPs resulted in a significant enhancement of blood circulation of DOX and IR820 in healthy mice, more studies must be done to further improve the NPs delivery. Loss of NPs during the transporting to tumors in plasma circulation often happens due to the binding of NPs to serum proteins known as surface opsonization, which results in the NPs being recognized and cleared by phagocytes or marcophages (161, 169). Modification of NPs surface with polyethylene glycol (PEG) can improve plasma circulation time and enhanced tumor uptake as compared to their non-PEGylated counterparts (165-167), which is possibly related to the surface charge of NPs and the protection of PEG to reduce albumin protein binding $(127,169)$. These reports indicated that modifying the NPs surface with PEG could be promising in enhancement of NPs delivery. Thus, one consideration to improve the NPs delivery is to formulate PEGylated PGMD NPs.

$4^{\text {th }}$. Another consideration is to formulate the NPs for targeting to achieve optimal delivery. As discussed before in the introduction section, antibody/ligand targeted NPs have better accumulation efficiency than their non-counjugated counterparts. The therapeutic potential of nanocarriers can be further magnified by tagging them with appropriate ligands that selectively interact with tumor cell membrane receptors. This method of tagging the drug delivery vehicle with a ligand and allowing it to specifically sequester in the targeted tumor is an example of active targeting and could confer greater specificity to different types of tumors. Therefore, PGMD NPs can be surface modified to conjugate different ligands or antibodies to further improve the specificity to tumor sites and uptake into cancer cells. 
References:

1.World Health Organization. The Global Burden of Disease: 2004 Update Geneva2008.

2.Liotta L, Petricoin E. Molecular profiling of human cancer. Nat Rev Genet. 2000 Oct;1(1):48-56.

3.Petricoin EF, Zoon KC, Kohn EC, Barrett JC, Liotta LA. Clinical proteomics: Translating benchside promise into bedside reality. Nat Rev Drug Discov. 2002 Sep;1(9):683-95.

4.Wang X, Wang YQ, Chen Z, Shin DM. Advances of Cancer Therapy by Nanotechnology. Cancer Res Treat. 2009 Mar;41(1):1-11.

5.Ehrlich P, Bolduan C. Collected studies on immunity. New York: J. Wiley \& sons; 1906.

6.Ferrari M. Cancer nanotechnology: Opportunities and challenges. Nature Reviews Cancer. 2005 Mar;5(3):161-71.

7.Srinivas PR, Barker P, Srivastava S. Nanotechnology in early detection of cancer. Lab Invest. 2002 May;82(5):657-62.

8.Henglein A. Small-Particle Research - Physicochemical Properties of Extremely Small Colloidal Metal and Semiconductor Particles. Chem Rev. 1989 Dec;89(8):1861-73.

9.Schmid G. Large Clusters and Colloids - Metals in the Embryonic State. Chem Rev. 1992 Dec;92(8):1709-27.

10.Niemeyer CM. Nanoparticles, proteins, and nucleic acids: Biotechnology meets materials science. Angew Chem Int Edit. 2001;40(22):4128-58.

11.Blanco E, Kessinger CW, Sumer BD, Gao J. Multifunctional Micellar Nanomedicine for Cancer Therapy. Exp Biol Med. 2009 Feb;234(2):123-31.

12.Park JW. Liposome-based drug delivery in breast cancer treatment. Breast Cancer Res. 2002;4(3):93-7.

13. Noble CO, Kirpotin DB, Hayes ME, Mamot C, Hong K, Park JW, et al. Development of ligand-targeted liposomes for cancer therapy. Expert Opin Ther Tar. 2004 Aug;8(4):335-53. 
14.Hood JD, Bednarski M, Frausto R, Guccione S, Reisfeld RA, Xiang R, et al. Tumor regression by targeted gene delivery to the neovasculature. Science. 2002 Jun 28;296(5577):2404-7.

15.Harisinghani MG, Barentsz J, Hahn PF, Deserno WM, Tabatabaei S, van de Kaa CH, et al. Noninvasive detection of clinically occult lymph-node metastases in prostate cancer. N Engl J Med. 2003 Jun 19;348(25):2491-9.

16.Lian T, Ho RJ. Trends and developments in liposome drug delivery systems. J Pharm Sci. 2001 Jun;90(6):667-80.

17.Bangham AD. Liposomes: the Babraham connection. Chem Phys Lipids. 1993 Sep;64(1-3):275-85.

18.Sapra P, Tyagi P, Allen TM. Ligand-targeted liposomes for cancer treatment. Curr Drug Deliv. 2005 Oct;2(4):369-81.

19.Huang A, Kennel SJ, Huang L. Interactions of Immunoliposomes with Target-Cells. Journal of Biological Chemistry. 1983;258(22):4034-40.

20.Labhasetwar V, Song CX, Humphrey W, Shebuski R, Levy RJ. Arterial uptake of biodegradable nanoparticles: Effect of surface modifications. Journal of Pharmaceutical Sciences. 1998 Oct;87(10):1229-34.

21.Jain RA. The manufacturing techniques of various drug loaded biodegradable poly(lactide-co-glycolide) (PLGA) devices. Biomaterials. 2000 Dec;21(23):2475-90.

22.Abdelwahed W, Degobert G, Stainmesse S, Fessi H. Freeze-drying of nanoparticles: Formulation, process and storage considerations. Advanced Drug Delivery Reviews. 2006 Dec 30;58(15):1688-713.

23.Elvassore N, Bertucco A, Caliceti P. Production of insulin-loaded poly(ethylene glycol)/poly(l-lactide) (PEG/PLA) nanoparticles by gas antisolvent techniques. Journal of Pharmaceutical Sciences. 2001 Oct;90(10):1628-36.

24.Galindo-Rodriguez S, Allemann E, Fessi H, Doelker E. Physicochemical parameters associated with nanoparticle formation in the salting-out, emulsification-diffusion, and nanoprecipitation methods. Pharm Res. 2004 Aug;21(8):1428-39.

25.Muller RH, Maassen S, Weyhers H, Specht F, Lucks JS. Cytotoxicity of magnetiteloaded polylactide, polylactide/glycolide particles and solid lipid nanoparticles. International Journal of Pharmaceutics. 1996 Jul 12;138(1):85-94. 
26.Leroux JC, Allemann E, DeJaeghere F, Doelker E, Gurny R. Biodegradable nanoparticles - From sustained release formulations to improved site specific drug delivery. Journal of Controlled Release. 1996 May;39(2-3):339-50.

27.Niwa T, Takeuchi H, Hino T, Kunou N, Kawashima Y. Preparations of Biodegradable Nanospheres of Water-Soluble and Insoluble Drugs with D,L-Lactide Glycolide Copolymer by a Novel Spontaneous Emulsification Solvent Diffusion Method, and the Drug Release Behavior. Journal of Controlled Release. 1993 May 27;25(1-2):89-98.

28.Nicolas J, Couvreur P. Synthesis of poly(alkyl cyanoacrylate)-based colloidal nanomedicines. Wiley Interdiscip Rev Nanomed Nanobiotechnol. 2009 JanFeb;1(1):111-27.

29.Moghimi SM, Hunter AC, Murray JC. Nanomedicine: current status and future prospects. The FASEB Journal. 2005 March 1, 2005;19(3):311-30.

30.Lavasanifar A, Samuel J, Kwon GS. Poly(ethylene oxide)-block-poly(L-amino acid) micelles for drug delivery. Advanced Drug Delivery Reviews. 2002 Feb 21;54(2):169-90.

31.Heller J. Ocular delivery using poly(ortho esters). Advanced Drug Delivery Reviews. 2005 Dec 13;57(14):2053-62.

32.Park ES, Maniar M, Shah JC. Biodegradable polyanhydride devices of cefazolin sodium, bupivacaine, and taxol for local drug delivery: preparation, and kinetics and mechanism of in vitro release. Journal of Controlled Release. 1998 Mar 2;52(1-2):17989.

33.Fernandez-Fernandez A, Manchanda R, McGoron AJ. Theranostic Applications of Nanomaterials in Cancer: Drug Delivery, Image-Guided Therapy, and Multifunctional Platforms. Appl Biochem Biotech. 2011 Dec;165(7-8):1628-51.

34.Guzman LA, Labhasetwar V, Song CX, Jang YS, Lincoff AM, Levy R, et al. Local intraluminal infusion of biodegradable polymeric nanoparticles - A novel approach for prolonged drug delivery after balloon angioplasty. Circulation. 1996 Sep 15;94(6):14418.

35.Lei TJ, Srinivasan S, Tang Y, Manchanda R, Nagesetti A, Fernandez-Fernandez A, et al. Comparing cellular uptake and cytotoxicity of targeted drug carriers in cancer cell lines with different drug resistance mechanisms. Nanomed-Nanotechnol. 2011 Jun; 7(3):324-32.

36.Acharya S, Sahoo SK. PLGA nanoparticles containing various anticancer agents and tumour delivery by EPR effect. Advanced Drug Delivery Reviews. 2011 Mar $18 ; 63(3): 170-83$. 
37.Panyam J, Labhasetwar V. Biodegradable nanoparticles for drug and gene delivery to cells and tissue. Adv Drug Deliv Rev. 2003 Feb 24;55(3):329-47.

38. Yoo HS, Oh JE, Lee KH, Park TG. Biodegradable nanoparticles containing doxorubicin-PLGA conjugate for sustained release. Pharmaceutical Research. 1999 Jul;16(7):1114-8.

39.Fonseca C, Simões S, Gaspar R. Paclitaxel-loaded PLGA nanoparticles: preparation, physicochemical characterization and in vitro anti-tumoral activity. Journal of Controlled Release. 2002;83(2):273-86.

40.Kim D-H, Martin DC. Sustained release of dexamethasone from hydrophilic matrices using PLGA nanoparticles for neural drug delivery. Biomaterials. 2006;27(15):3031-7.

41.Yuan F, Dellian M, Fukumura D, Leunig M, Berk DA, Torchilin VP, et al. Vascular permeability in a human tumor xenograft: molecular size dependence and cutoff size. Cancer Research. 1995 Sep 1;55(17):3752-6.

42.Hobbs SK, Monsky WL, Yuan F, Roberts WG, Griffith L, Torchilin VP, et al. Regulation of transport pathways in tumor vessels: role of tumor type and microenvironment. Proc Natl Acad Sci U S A. 1998 Apr 14;95(8):4607-12.

43.Gottesman MM. Mechanisms of cancer drug resistance. Annu Rev Med. 2002;53:61527.

44.Vasir JK, Reddy MK, Labhasetwar VD. Nanosystems in drug targeting: Opportunities and challenges. Curr Nanosci. 2005 Jan;1(1):47-64.

45.Chang HR. Trastuzumab-Based Neoadjuvant Therapy in Patients With HER2-Positive Breast Cancer. Cancer-Am Cancer Soc. 2010 Jun 15;116(12):2856-67.

46.Glass B, Ziepert M, Reiser M, Freund M, Trumper L, Metzner B, et al. High-dose therapy followed by autologous stem-cell transplantation with and without rituximab for primary treatment of high-risk diffuse large B-cell lymphoma. Ann Oncol. 2010 Nov;21(11):2255-61.

47.Garnett MC. Targeted drug conjugates: principles and progress. Advanced Drug Delivery Reviews. 2001 Dec 17;53(2):171-216.

48.Funaro A, Horenstein AL, Santoro P, Cinti C, Gregorini A, Malavasi F. Monoclonal antibodies and therapy of human cancers. Biotechnol Adv. 2000 Aug;18(5):385-401.

49.Simpson CR, Kohl M, Essenpreis M, Cope M. Near-infrared optical properties of ex vivo human skin and subcutaneous tissues measured using the Monte Carlo inversion technique. Phys Med Biol. 1998 Sep;43(9):2465-78. 
50. Weissleder R, Tung $\mathrm{CH}$, Mahmood U, Bogdanov A, Jr. In vivo imaging of tumors with protease-activated near-infrared fluorescent probes. Nat Biotechnol. 1999 Apr;17(4):375-8.

51.Fickweiler S, Szeimies RM, Baumler W, Steinbach P, Karrer S, Goetz AE, et al. Indocyanine green: intracellular uptake and phototherapeutic effects in vitro. J Photochem Photobiol B. 1997 Apr;38(2-3):178-83.

52.Zaidi SI, Agarwal R, Eichler G, Rihter BD, Kenney ME, Mukhtar H. Photodynamic effects of new silicon phthalocyanines: in vitro studies utilizing rat hepatic microsomes and human erythrocyte ghosts as model membrane sources. Photochem Photobiol. 1993;58(2):204-10.

53.Gaboury L, Villeneuve, L., Giasson, R., Li, T., Gupta, A.K., , inventor Novel rhodamine derivatives for photodynamic therapy of cancer and in vitro purging of the leukemias. United States 1998.

54.Abels C, Fickweiler S, Weiderer P, Bäumler W, Hofstädter F, Landthaler M, et al. Indocyanine green (ICG) and laser irradiation induce photooxidation. Archives of Dermatological Research. 2000;292(8):404-11.

55.Tang Y, McGoron AJ. Combined effects of laser-ICG photothermotherapy and doxorubicin chemotherapy on ovarian cancer cells. Journal of Photochemistry and Photobiology B: Biology. 2009;97(3):138-44.

56.Dorshow RB, Bugaj JE, Burleigh BD, Duncan JR, Johnson MA, Jones WB. Noninvasive Fluorescence Detection of Hepatic and Renal Function. Journal of Biomedical Optics. 1998;3(3):340-5.

57.Diederich CJ. Thermal ablation and high-temperature thermal therapy: overview of technology and clinical implementation. Int J Hyperthermia. 2005 Dec;21(8):745-53.

58.Issels RD, Prenninger SW, Nagele A, Boehm E, Sauer H, Jauch KW, et al. Ifosfamide Plus Etoposide Combined with Regional Hyperthermia in Patients with Locally Advanced Sarcomas - a Phase-Ii Study. J Clin Oncol. 1990 Nov;8(11):1818-29.

59.Wust P, Stahl H, Dieckmann K, Scheller S, Löffel J, Riess H, et al. Local hyperthermia of N2N3 cervical lymph node metastases: Correlation of technical/thermal parameters and response. International Journal of Radiation Oncology*Biology*Physics. 1996;34(3):635-46.

60.Rau B, Wust P, Tilly W, Gellermann J, Harder C, Riess H, et al. Preoperative radiochemotherapy in locally advanced or recurrent rectal cancer: Regional radiofrequency hyperthermia correlates with clinical parameters. Int J Radiat Oncol. 2000 Sep 1;48(2):381-91. 
61.Hamazoe R, Maeta M, Kaibara N. Intraperitoneal Thermochemotherapy for Prevention of Peritoneal Recurrence of Gastric-Cancer - Final Results of a Randomized Controlled-Study. Cancer-Am Cancer Soc. 1994 Apr 15;73(8):2048-52.

62.Hafstrom L, Rudenstam CM, Blomquist E, Ingvar C, Jonsson PE, Lagerlof B, et al. Regional Hyperthermic Perfusion with Melphalan after Surgery for Recurrent MalignantMelanoma of the Extremities. J Clin Oncol. 1991 Dec;9(12):2091-4.

63.Park H, Yang J, Lee J, Haam S, Choi I-H, Yoo K-H. Multifunctional Nanoparticles for Combined Doxorubicin and Photothermal Treatments. ACS Nano. 2009 2009/10/27;3(10):2919-26.

64.Day ES, Morton JG, West JL. Nanoparticles for Thermal Cancer Therapy. Journal of Biomechanical Engineering. 2009;131(7):074001-5.

65.Ito A, Shinkai M, Honda H, Kobayashi T. Medical application of functionalized magnetic nanoparticles. J Biosci Bioeng. 2005 Jul;100(1):1-11.

66.Gupta AK, Gupta M. Synthesis and surface engineering of iron oxide nanoparticles for biomedical applications. Biomaterials. 2005 Jun;26(18):3995-4021.

67.Huber DL. Synthesis, properties, and applications of iron nanoparticles. Small. 2005 May;1(5):482-501.

68.Desmettre T, Devoisselle JM, Mordon S. Fluorescence properties and metabolic features of indocyanine green (ICG) as related to angiography. Surv Ophthalmol. 2000 Jul-Aug;45(1):15-27.

69.Saxena V, Sadoqi M, Shao J. Indocyanine green-loaded biodegradable nanoparticles: preparation, physicochemical characterization and in vitro release. International Journal of Pharmaceutics. 2004 Jul 8;278(2):293-301.

70.Manchanda R, Fernandez-Fernandez A, Nagesetti A, McGoron AJ. Preparation and characterization of a polymeric (PLGA) nanoparticulate drug delivery system with simultaneous incorporation of chemotherapeutic and thermo-optical agents. Colloid Surface B. 2010 Jan 1;75(1):260-7.

71.Migneco F, Huang Y-C, Birla RK, Hollister SJ. Poly(glycerol-dodecanoate), a biodegradable polyester for medical devices and tissue engineering scaffolds. Biomaterials. 2009;30(33):6479-84.

72.Sundback CA, Shyu JY, Wang Y, Faquin WC, Langer RS, Vacanti JP, et al. Biocompatibility analysis of poly(glycerol sebacate) as a nerve guide material. Biomaterials. 2005 Sep;26(27):5454-64. 
73.Shet MS, Fisher CW, Holmans PL, Estabrook RW. The omega-hydroxylation of lauric acid: Oxidation of 12-hydroxylauric acid to dodecanedioic acid by a purified recombinant fusion protein containing P450 4A1 and NADPH-P450 reductase. Arch Biochem Biophys. 1996 Jun 1;330(1):199-208.

74.Gieseler F, Biersack H, Brieden T, Manderscheid J, Nüßler V. Cytotoxicity of anthracyclines: Correlation with cellular uptake, intracellular distribution and DNA binding. Annals of Hematology. 1994;69(1):S13-S7.

75.Belloc F, Lacombe F, Domain P, Lopez F, Bernard P, Boisseau MR, et al. Intercalation of anthracyclines into living cell DNA analyzed by flow cytometry. Cytometry. 1992;13(8):880-5.

76.Singal PK, Iliskovic N. Doxorubicin-induced cardiomyopathy. New Engl J Med. 1998 Sep 24;339(13):900-5.

77.Yoo HS, Park TG. Biodegradable polymeric micelles composed of doxorubicin conjugated PLGA-PEG block copolymer. Journal of Controlled Release. 2001;70(12):63-70.

78.Lee ES, Na K, Bae YH. Doxorubicin loaded $\mathrm{pH}$-sensitive polymeric micelles for reversal of resistant MCF-7 tumor. Journal of Controlled Release. 2005;103(2):405-18.

79.Fernandez-Fernandez A, Manchanda R, Lei T, Carvajal DA, Tang Y, Kazmi SZ, et al. Comparative study of the optical and heat generation properties of IR820 and indocyanine green. Mol Imaging. 2012 Apr;11(2):99-113.

80.Masotti A, Vicennati P, Boschi F, Calderan L, Sbarbati A, Ortaggi G. A novel nearinfrared indocyanine dye-polyethylenimine conjugate allows DNA delivery imaging in vivo. Bioconjug Chem. 2008 May;19(5):983-7.

81.Magenheim B, Levy MY, Benita S. A New in-Vitro Technique for the Evaluation of Drug-Release Profile from Colloidal Carriers - Ultrafiltration Technique at Low-Pressure. International Journal of Pharmaceutics. 1993 Jun 21;94(1-3):115-23.

82.Chen Y, Mcculloch RK, Gray BN. Synthesis of Albumin-Dextran Sulfate Microspheres Possessing Favorable Loading and Release Characteristics for the Anticancer Drug Doxorubicin. Journal of Controlled Release. 1994 Aug;31(1):49-54.

83. Calvo P, RemunanLopez C, VilaJato JL, Alonso MJ. Chitosan and chitosan ethylene oxide propylene oxide block copolymer nanoparticles as novel carriers for proteins and vaccines. Pharmaceutical Research. 1997 Oct;14(10):1431-6.

84.Tamada JA, Langer R. Erosion Kinetics of Hydrolytically Degradable Polymers. P Natl Acad Sci USA. 1993 Jan 15;90(2):552-6. 
85. Vert M, Christel P, Chabot F, Leray J. Macromolecular materials. In: Hastings GW, P D, editors.; Boca Raton, FL: CRC Press; 1984. p. p. 119

86.Li SM, Garreau H, Vert M. Structure Property Relationships in the Case of the Degradation of Massive Aliphatic Poly-(Alpha-Hydroxy Acids) in Aqueous-Media .1. Poly(Dl-Lactic Acid). J Mater Sci-Mater M. 1990 Oct;1(3):123-30.

87.Li SM, GirodHolland S, Vert M. Hydrolytic degradation of poly(DL-lactic acid) in the presence of caffeine base. Journal of Controlled Release. 1996 Jun;40(1-2):41-53.

88. Burkersroda Fv, Schedl L, Göpferich A. Why degradable polymers undergo surface erosion or bulk erosion. Biomaterials. 2002;23(21):4221-31.

89. Greco O, Marples B, Joiner MC, Scott SD. How to overcome (and exploit) tumor hypoxia for targeted gene therapy. J Cell Physiol. 2003 Dec;197(3):312-25.

90.Brown JM. Exploiting the hypoxic cancer cell: mechanisms and therapeutic strategies. Mol Med Today. 2000 Apr;6(4):157-62.

91.Matthews NE, Adams MA, Maxwell LR, Gofton TE, Graham CH. Nitric oxidemediated regulation of chemosensitivity in cancer cells. J Natl Cancer I. 2001 Dec 19;93(24):1879-85.

92.Vaupel P, Kelleher DK, Hockel M. Oxygenation status of malignant tumors: Pathogenesis of hypoxia and significance for tumor therapy. Semin Oncol. 2001 Apr;28(2):29-35.

93. Yokoi K, Fidler IJ. Hypoxia Increases Resistance of Human Pancreatic Cancer Cells to Apoptosis Induced by Gemcitabine. Clinical Cancer Research. 2004 April 1, 2004;10(7):2299-306.

94.Forsythe JA, Jiang BH, Iyer NV, Agani F, Leung SW, Koos RD, et al. Activation of vascular endothelial growth factor gene transcription by hypoxia-inducible factor 1. Mol Cell Biol. 1996 Sep;16(9):4604-13.

95.Sullivan R, Pare GC, Frederiksen LJ, Semenza GL, Graham CH. Hypoxia-induced resistance to anticancer drugs is associated with decreased senescence and requires hypoxia-inducible factor-1 activity. Mol Cancer Ther. 2008 Jul;7(7):1961-73.

96.Vaupel P. Tumor microenvironmental physiology and its implications for radiation oncology. Semin Radiat Oncol. 2004 Jul;14(3):198-206.

97.Kim JW, Tchernyshyov I, Semenza GL, Dang CV. HIF-1-mediated expression of pyruvate dehydrogenase kinase: A metabolic switch required for cellular adaptation to hypoxia. Cell Metabolism. 2006 Mar;3(3):177-85. 
98. Moon EJ, Sonveaux P, Porporato PE, Danhier P, Gallez B, Batinic-Haberle I, et al. NADPH oxidase-mediated reactive oxygen species production activates hypoxiainducible factor-1 (HIF-1) via the ERK pathway after hyperthermia treatment. Proc Natl Acad Sci U S A. 2010 Nov 23;107(47):20477-82.

99. Goyal P, Weissmann N, Grimminger F, Hegel C, Bader L, Rose F, et al. Upregulation of $\mathrm{NAD}(\mathrm{P}) \mathrm{H}$ oxidase 1 in hypoxia activates hypoxia-inducible factor 1 via increase in reactive oxygen species. Free Radical Biology and Medicine. 2004;36(10):1279-88.

100.Chandel NS, McClintock DS, Feliciano CE, Wood TM, Melendez JA, Rodriguez AM, et al. Reactive Oxygen Species Generated at Mitochondrial Complex III Stabilize Hypoxia-inducible Factor-1 $\alpha$ during Hypoxia: A MECHANISM OF O2 SENSING. Journal of Biological Chemistry. 2000 August 18, 2000;275(33):25130-8.

101.Katschinski DM, Le L, Heinrich D, Wagner KF, Hofer T, Schindler SG, et al. Heat induction of the unphosphorylated form of hypoxia-inducible factor-1 alpha is dependent on heat shock protein-90 activity. Journal of Biological Chemistry. 2002 Mar 15;277(11):9262-7.

102.Nishizawa J, Nakai A, Matsuda K, Komeda M, Ban T, Nagata K. Reactive oxygen species play an important role in the activation of heat shock factor 1 in ischemicreperfused heart. Circulation. 1999 Feb 23;99(7):934-41.

103.Li GC, Mivechi NF, Weitzel G. Heat shock proteins, thermotolerance, and their relevance to clinical hyperthermia. Int J Hyperther. 1995;11(4):459-88.

104.Hildebrandt B, Wust P, Ahlers O, Dieing A, Sreenivasa G, Kerner T, et al. The cellular and molecular basis of hyperthermia. Crit Rev Oncol Hematol. 2002 Jul;43(1):33-56.

105.Madamanchi NR, Li S, Patterson C, Runge MS. Reactive Oxygen Species Regulate Heat-Shock Protein 70 via the JAK/STAT Pathway. Arteriosclerosis, Thrombosis, and Vascular Biology. 2001 March 1, 2001;21(3):321-6.

106.Tang Y, McGoron AJ. The role of temperature increase rate in combinational hyperthermia chemotherapy treatment. 2010:75650C-C.

107.Srinivasan S, Manchanda R, Fernandez-Fernandez A, Lei T, McGoron AJ. Nearinfrared fluorescing IR820-chitosan conjugate for multifunctional cancer theranostic applications. J Photochem Photobiol B. 2012 Dec 28;119C:52-9.

108.Manchanda R, Fernandez-Fernandez A, Carvajal DA, Lei T, Tang Y, McGoron AJ. Nanoplexes for cell imaging and hyperthermia: in vitro studies. J Biomed Nanotechnol. 2012 Aug;8(4):686-94. 
109.Prajapati SI, Martinez CO, Bahadur AN, Wu IQ, Zheng W, Lechleiter JD, et al. Near-Infrared Imaging of Injured Tissue in Living Subjects Using IR-820. Molecular imaging. 2009 Jan-Feb;8(1):45-54.

110.Sapareto SA, Dewey WC. Thermal dose determination in cancer therapy. International Journal of Radiation Oncology*Biology*Physics. 1984;10(6):787-800.

111.Monks A, Scudiero D, Skehan P, Shoemaker R, Paull K, Vistica D, et al. Feasibility of a High-Flux Anticancer Drug Screen Using a Diverse Panel of Cultured Human Tumor-Cell Lines. J Natl Cancer I. 1991 Jun 5;83(11):757-66.

112.Rajagopalan R, Uetrecht P, Bugaj JE, Achilefu SA, Dorshow RB. Stabilization of the Optical Tracer Agent Indocyanine Green Using Noncovalent Interactions. Photochemistry and Photobiology. 2000;71(3):347-50.

113.Wolfensohn S, Lloyd M. Handbook of Laboratory Animal Management and Welfare. Oxford, UK: Wiley-Blackwell; 2003.

114.Fournier RL. Basic Transport Phenomena in Biomedical Engineering. New York, NY: Taylor \& Francis; 2007.

115.Truskey GA, Yuan F, Katz DF. Transport Phenomena in Biological Systems. Upper Saddle River, NJ: Pearson Prentice Hall; 2009.

116.Bauer L. Applied Clinical Pharmacokinetics. New York, NY: McGraw Hill; 2008.

117.Macon MB, Villanueva LR, Tatum-Gibbs K, Zehr RD, Strynar MJ, Stanko JP, et al. Prenatal perfluorooctanoic acid exposure in CD-1 mice: low-dose developmental effects and internal dosimetry. Toxicol Sci. $2011 \mathrm{Jul} ; 122(1): 134-45$.

118.Achilefu S, Dorshow R. Dynamic and Continuous Monitoring of Renal and Hepatic Functions with Exogenous Markers. In: Krause W, editor. Contrast Agents II: Springer Berlin Heidelberg; 2002. p. 31-72.

119.Gulyaev AE, Gelperina SE, Skidan IN, Antropov AS, Kivman GY, Kreuter J. Significant Transport of Doxorubicin into the Brain with Polysorbate 80-Coated Nanoparticles. Pharmaceutical Research. 1999;16(10):1564-9.

120. Rahman A, Carmichael D, Harris M, Roh JK. Comparative pharmacokinetics of free doxorubicin and doxorubicin entrapped in cardiolipin liposomes. Cancer Research. 1986 May;46(5):2295-9.

121.Gabizon AA, Barenholz Y, Bialer M. Prolongation of the Circulation Time of Doxorubicin Encapsulated in Liposomes Containing a Polyethylene Glycol-Derivatized 
Phospholipid: Pharmacokinetic Studies in Rodents and Dogs. Pharmaceutical Research. 1993;10(5):703-8.

122.Saxena V, Sadoqi M, Shao J. Polymeric nanoparticulate delivery system for Indocyanine green: Biodistribution in healthy mice. Int J Pharm. 2006;308(1-2):200-4.

123.Misra R, Sahoo SK. Intracellular trafficking of nuclear localization signal conjugated nanoparticles for cancer therapy. European Journal of Pharmaceutical Sciences. 2010 Jan $31 ; 39(1-3): 152-63$.

124.Qaddoumi MG, Gukasyan HJ, Davda J, Labhasetwar V, Kim KJ, Lee VH. Clathrin and caveolin-1 expression in primary pigmented rabbit conjunctival epithelial cells: role in PLGA nanoparticle endocytosis. Molecular Vision. 2003 Oct 15;9:559-68.

125.Kaplowitz N, Kuhlenkamp J, Clifton G. Hepatic glutathione S-transferases: identification by gel filtration and in vitro inhibition by organic anions. Proceedings of the Society for Experimental Biology and Medicine. 1975 May;149(1):234-7.

126.Kaplowitz N, Kuhlenkamp J, Clifton G. Hepatic glutathione S-transferases: identification by gel filtration and in vitro inhibition by organic anions. Proc Soc Exp Biol Med. 1975 May;149(1):234-7.

127.Li SD, Huang L. Pharmacokinetics and biodistribution of nanoparticles. Molecular Pharmaceutics. 2008 Jul-Aug;5(4):496-504.

128.Reddy LH, Murthy RS. Pharmacokinetics and biodistribution studies of Doxorubicin loaded poly(butyl cyanoacrylate) nanoparticles synthesized by two different techniques. Biomed Pap Med Fac Univ Palacky Olomouc Czech Repub. 2004 Dec;148(2):161-6.

129.Gustafson DL, Rastatter JC, Colombo T, Long ME. Doxorubicin pharmacokinetics: Macromolecule binding, metabolism, and excretion in the context of a physiologic model. J Pharm Sci. 2002;91(6):1488-501.

130.Vroman I, Tighzert L. Biodegradable Polymers. Materials. 2009;2(2):307-44.

131.Löfgren A, Albertsson A-C, Dubois P, Jérôme R. Recent Advances in Ring-Opening Polymerization of Lactones and Related Compounds. Journal of Macromolecular Science, Part C. 1995 1995/08/01;35(3):379-418.

132.Padilla De Jesús OL, Ihre HR, Gagne L, Fréchet JMJ, Szoka FC. Polyester Dendritic Systems for Drug Delivery Applications: In Vitro and In Vivo Evaluation. Bioconjug Chem. 2002 2002/05/01;13(3):453-61. 
133.Moghimi SM, Hunter AC, Murray JC. Long-circulating and target-specific nanoparticles: theory to practice. Pharmacol Rev. 2001 Jun;53(2):283-318.

134.Cheng J, Teply BA, Sherifi I, Sung J, Luther G, Gu FX, et al. Formulation of functionalized PLGA-PEG nanoparticles for in vivo targeted drug delivery. Biomaterials. 2007;28(5):869-76.

135.Zolnik BS, Leary PE, Burgess DJ. Elevated temperature accelerated release testing of PLGA microspheres. Journal of Controlled Release. 2006;112(3):293-300.

136.Chilkoti A, Dreher MR, Meyer DE, Raucher D. Targeted drug delivery by thermally responsive polymers. Advanced Drug Delivery Reviews. 2002;54(5):613-30.

137.Bikram M, West JL. Thermo-responsive systems for controlled drug delivery. Expert Opinion on Drug Delivery. 2008 2008/10/01;5(10):1077-91.

138.Meyer DE, Shin BC, Kong GA, Dewhirst MW, Chilkoti A. Drug targeting using thermally responsive polymers and local hyperthermia. Journal of Controlled Release. $2001 ; 74(1-3): 213-24$

139.Zhang X-Z, Zhuo R-X, Cui J-Z, Zhang J-T. A novel thermo-responsive drug delivery system with positive controlled release. International Journal of Pharmaceutics. 2002;235(1-2):43-50.

140.Gillies ER, Fréchet JMJ. pH-Responsive Copolymer Assemblies for Controlled Release of Doxorubicin. Bioconjugate Chemistry. 2005 2005/03/01;16(2):361-8.

141.Vaupel P, Kallinowski F, Okunieff P. Blood-Flow, Oxygen and Nutrient Supply, and Metabolic Microenvironment of Human-Tumors - a Review. Cancer Research. 1989 Dec 1;49(23):6449-65.

142.Engin K, Leeper DB, Cater JR, Thistlethwaite AJ, Tupchong L, Mcfarlane JD. Extracellular $\mathrm{Ph}$ Distribution in Human Tumors. Int J Hyperther. 1995 MarApr;11(2):211-6.

143.Tang Y, Lei T, Manchanda R, Nagesetti A, Fernandez-Fernandez A, Srinivasan S, et al. Simultaneous delivery of chemotherapeutic and thermal-optical agents to cancer cells by a polymeric (PLGA) nanocarrier: an in vitro study. Pharm Res. 2010 Oct;27(10):224253.

144.Panyam J, Zhou WZ, Prabha S, Sahoo SK, Labhasetwar V. Rapid endo-lysosomal escape of poly(DL-lactide-co-glycolide) nanoparticles: implications for drug and gene delivery. FASEB J. 2002 Aug;16(10):1217-26. 
145.Zhang K, Fang HF, Chen ZY, Taylor JSA, Wooley KL. Shape effects of nanoparticles conjugated with cell-penetrating peptides (HIV Tat PTD) on CHO cell uptake. Bioconjugate Chemistry. 2008 Sep;19(9):1880-7.

146.Chithrani BD, Chan WCW. Elucidating the mechanism of cellular uptake and removal of protein-coated gold nanoparticles of different sizes and shapes. Nano Lett. 2007 Jun; 7(6):1542-50.

147.Liang M, Lin IC, Whittaker MR, Minchin RF, Monteiro MJ, Toth I. Cellular Uptake of Densely Packed Polymer Coatings on Gold Nanoparticles. ACS Nano. 2010 Jan;4(1):403-13.

148.Chung TH, Wu SH, Yao M, Lu CW, Lin YS, Hung Y, et al. The effect of surface charge on the uptake and biological function of mesoporous silica nanoparticles 3T3-L1 cells and human mesenchymal stem cells. Biomaterials. 2007 Jul;28(19):2959-66.

149.Dewhirst MW, Viglianti BL, Lora-Michiels M, Hanson M, Hoopes PJ. Basic principles of thermal dosimetry and thermal thresholds for tissue damage from hyperthermia. International journal of hyperthermia : the official journal of European Society for Hyperthermic Oncology, North American Hyperthermia Group. 2003 MayJun;19(3):267-94.

150.Moon EJ, Sonveaux P, Porporato PE, Danhier P, Gallez B, Batinic-Haberle I, et al. NADPH oxidase-mediated reactive oxygen species production activates hypoxiainducible factor-1 (HIF-1) via the ERK pathway after hyperthermia treatment. Proceedings of the National Academy of Sciences. 2010 November 23, 2010;107(47):20477-82.

151.Mansfield KD, Guzy RD, Pan Y, Young RM, Cash TP, Schumacker PT, et al. Mitochondrial dysfunction resulting from loss of cytochrome c impairs cellular oxygen sensing and hypoxic HIF-alpha activation. Cell Metab. 2005 Jun;1(6):393-9.

152.Semenza GL. Targeting HIF-1 for cancer therapy. Nat Rev Cancer. 2003 Oct;3(10):721-32.

153. Nevaldine B, Longo JA, Hahn PJ. Hyperthermia inhibits the repair of DNA doublestrand breaks induced by ionizing radiation as determined by pulsed-field gel electrophoresis. Int J Hyperther. 1994;10(3):381-8.

154.Jain RK. Determinants of Tumor Blood-Flow - a Review. Cancer Research. 1988 May 15;48(10):2641-58.

155.Sullivan R, Paré GC, Frederiksen LJ, Semenza GL, Graham CH. Hypoxia-induced resistance to anticancer drugs is associated with decreased senescence and requires 
hypoxia-inducible factor-1 activity. Molecular Cancer Therapeutics. 2008 July 1, 2008;7(7):1961-73.

156.Wartenberg M, Gronczynska S, Bekhite MM, Saric T, Niedermeier W, Hescheler J, et al. Regulation of the multidrug resistance transporter P-glycoprotein in multicellular prostate tumor spheroids by hyperthermia and reactive oxygen species. Int J Cancer. 2005 Jan 10;113(2):229-40.

157.Harada H, Kizaka-Kondoh S, Li G, Itasaka S, Shibuya K, Inoue M, et al. Significance of HIF-1-active cells in angiogenesis and radioresistance. Oncogene. 2007 Nov 29;26(54):7508-16.

158.Brown LM, Cowen RL, Debray C, Eustace A, Erler JT, Sheppard FCD, et al. Reversing Hypoxic Cell Chemoresistance in Vitro Using Genetic and Small Molecule Approaches Targeting Hypoxia Inducible Factor-1. Molecular Pharmacology. 2006 February 1, 2006;69(2):411-8.

159.Song X, Liu X, Chi W, Liu Y, Wei L, Wang X, et al. Hypoxia-induced resistance to cisplatin and doxorubicin in non-small cell lung cancer is inhibited by silencing of HIF$1 \alpha$ gene. Cancer Chemother Pharmacol. 2006 2006/12/01;58(6):776-84.

160.Liu D, Mori A, Huang L. Role of liposome size and RES blockade in controlling biodistribution and tumor uptake of GM1-containing liposomes. Biochim Biophys Acta. 1992 Feb 17;1104(1):95-101.

161.Moghimi SM, Hunter AC, Andresen TL. Factors Controlling Nanoparticle Pharmacokinetics: An Integrated Analysis and Perspective. Annual Review of Pharmacology and Toxicology, Vol 52. 2012;52:481-503.

162.Fernandez-Fernandez A, Manchanda R, Carvajal DA, Lei T, McGoron AJ. Covalent IR820-PEG diamine conjugates: characterization and in vivo biodistribution. 2013:859605-.

163.Gabizon A, Catane R, Uziely B, Kaufman B, Safra T, Cohen R, et al. Prolonged circulation time and enhanced accumulation in malignant exudates of doxorubicin encapsulated in polyethylene-glycol coated liposomes. Cancer Research. 1994 Feb 15;54(4):987-92.

164.Gabizon A, Shmeeda H, Barenholz Y. Pharmacokinetics of Pegylated Liposomal Doxorubicin: Review of Animal and Human Studies. Clinical Pharmacokinetics. 2003;42(5):419-36.

165.Panagi Z, Beletsi A, Evangelatos G, Livaniou E, Ithakissios DS, Avgoustakis K. Effect of dose on the biodistribution and pharmacokinetics of PLGA and PLGA-mPEG nanoparticles. International Journal of Pharmaceutics. 2001;221(1-2):143-52. 
166.Gref R, Lück M, Quellec P, Marchand M, Dellacherie E, Harnisch S, et al. 'Stealth' corona-core nanoparticles surface modified by polyethylene glycol (PEG): influences of the corona (PEG chain length and surface density) and of the core composition on phagocytic uptake and plasma protein adsorption. Colloids and Surfaces B: Biointerfaces. 2000;18(3-4):301-13.

167.Zahr AS, Davis CA, Pishko MV. Macrophage Uptake of Core-Shell Nanoparticles Surface Modified with Poly(ethylene glycol). Langmuir. 2006 2006/09/01;22(19):817885.

168.Maeda H, Wu J, Sawa T, Matsumura Y, Hori K. Tumor vascular permeability and the EPR effect in macromolecular therapeutics: a review. J Controlled Release. 2000;65(1-2):271-84.

169.Albanese A, Tang PS, Chan WCW. The Effect of Nanoparticle Size, Shape, and Surface Chemistry on Biological Systems. Annu Rev Biomed Eng. 2012;14:1-16. 
VITA

TINGJUN LEI

Born, Nanning, Guangxi, China

2002-2006

B.E., Biomedical Engineering, Jiangsu University,

Zhenjiang, China

$2007-2013$

Doctoral Candidate, Biomedical Engineering

Florida International University

Miami, Florida

\section{PUBLICATIONS AND PRESENTATIONS (ABRIDGED LIST)}

Lei T, Srinivasan S, Tang Y, Manchanda R, Nagesetti A, Fernandez-Fernandez A, McGoron A.J. Comparing Cellular Uptake and Cytotoxicity of Targeted Drug Carriers in Cancer Cell Lines with Different Drug Resistance Mechanisms. Nanomedicine. 2011 Jun;7(3):324-32.

Srinivasan, S., Manchanda, R., Fernandez-Fernandez, A., Lei, T. \& McGoron, A. J. Near-infrared fluorescing IR820-chitosan conjugate for multifunctional cancer theranostic applications. Journal of Photochemistry and Photobiology B: Biology 119, 52-59, doi:http://dx.doi.org/10.1016/j.jphotobiol.2012.12.008 (2013).

Liu C, Lei T, Ino K, Matsue T, Tao N, Li CZ. Real-time monitoring biomarker expression of carcinoma cells by surface plasmon resonance biosensors. Chemical communications. DOI: 10.1039/c2cc34853e.

Manchanda R., Fernandez-Fernandez A., Carvajal D.A., Lei T, Tang Y, McGoron A.J., Nanoplexes for Cell Imaging and Hyperthermia: In Vitro Studies. J Biomed Nanotechnol. 2012 Aug;8(4):686-94.

Fernandez-Fernandez A, Manchanda R, Lei T, Carvajal DA, Tang Y, Zahid Raza Kazmi S, McGoron AJ, Comparative Study of the Optical and Heat Generation Properties of IR820 and Indocyanine Green. Mol Imaging. 2011 Sep 16;:1-10 21924114

Tang, Y., Lei T., Manchanda R., Nagesetti A., Fernandez- Fernandez A., Srinivasan S, McGoron A.J.. (2010). Simultaneous Delivery of Chemotherapeutic and Thermal-Optical Agents to Cancer Cells by a Polymeric (PLGA) Nanocarrier: an In Vitro Study. Pharmaceutical Research, 27, 2242-2253. 
Zhu W, Yang C, Lei T, Gao P, "The investigations of the portable multi-functional realtime physiological parameters monitor which is based on the Ling-Yang SCM" ("Electronic Products (China)", 2005, 60 63).

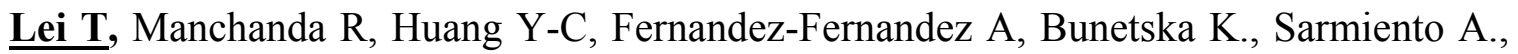
Milera A., McGoron A.J., Near-infrared imaging loaded polymericnanoparticles: In vitro and In vivo studies. SPIE, 2013, San Francisco.

Lei T, Fernandez-Fernandez A, Tang Y, Carvajal D, Manchanda R, Kazmi S.Z.R, McGoron A.J. A Comparative Study of IR-820 and Indocyanine Green (ICG); J Nucl Med. 2010; 51 (Supplement 2):225.

Lei T, Srinivasan S, Tang Y, Manchanda R, Fernandez-Fernandez A, McGoron A.J. Targeted Delivery of Doxorubicin by PLGA Nanoparticles Increases Drug Uptake in Cancer Cell Lines. IFMBE Proceedings 2010; 32:224-28.

Fernandez-Fernandez A, Manchanda R, Carvajal, D.A., Lei T., McGoron A.J. Covalent IR820-PEG diamine conjugates: characterization and in vivo biodistribution. SPIE 2013, San Francisco.

McGoron A.J., Srinivasan S., Lei T, Tang Y., Manchanda R. Combined photothermal therapy and chemotherapy in cancer using HER-2 targeted PLGA nanoparticles. SPIE 2013, San Francisco.

Manchanda R, Lei T, Huang Y-C, McGoron A.J. Co-delivery of near-infrared (NIR) imaging and chemotherapeutic agent using polymeric nanoparticles; J Nucl Med. 2012; 53 (Supplement 1):1573

Fernandez-Fernandez A, Manchanda R, Srinivasan S, Lei T, Carvajal D, McGoron A.J. IR820-chitosan conjugates for imaging and hyperthermia; J Nucl Med. 2012; 53 (Supplement 1):1172

Manchanda R, Lei T, Tang Y, Fernandez-Fernandez A, McGoron AJ. Cellular uptake and cytotoxicity of a novel ICG-DOX-PLGA dual agent polymer nanoparticles delivery system. IFMBE Proceedings 2010; 32:228-32.

Lei T, Manchanda R, Fernandez-Fernandez A, Huang Y-C, Bunetska K, Milera A, Sarmiento A, McGoron A.J. In vitro and In vivo studies of near-infrared imaging (NIR) loaded polymeric Nanoparticles. NanoFlorida 2012: The 4th Annual NanoScience Technology Symposium, Tampa, FL.

Lei T, Manchanda R, Huang Y-C, McGoron A.J. Thermal and $\mathrm{pH}$ sensitive multifunctional polymeric nanoparticles for cancer therapy. Biomedical Engineering Society 2012 Annual Meeting, Oct 24-27, 2012, Atlanta, GA. 